\title{
0. Introduction
}

Nonlinear dynamical systems are not only of intrinsic interest, but are also important in the study of nonlinear evolution equations, as well as of the geometry of domains in Banach spaces. For example, nonlinear nonexpansive semigroups find application in the theory of differential equations and can be viewed as nonlinear analogs of the classical linear contraction semigroups. See, for instance, [15, 11, 13, 59]. Another important class of nonlinear semigroups consists of semigroups of holomorphic self-mappings. Such semigroups occur in several diverse fields, including, for example, the theory of Markov stochastic branching processes [36, 74], Krein spaces [83-85], fixed point theory [51], the geometry of complex Banach spaces [9, 82], control theory and optimization [38]. These semigroups can be considered natural nonlinear analogs of the semigroups generated by bounded linear operators. The two distinct classes of nonlinear semigroups are related by the fact that holomorphic self-mappings are nonexpansive with respect to Schwarz-Pick pseudometrics.

For the finite dimensional case, M. Abate proved in [3] that each continuous semigroup of holomorphic mappings is everywhere differentiable with respect to its parameter, i.e., it is generated by a holomorphic mapping. In addition, he established a criterion for a holomorphic mapping to be a generator of a one-parameter semigroup. A parametric representation of such generators can be found in [5]. Earlier, in the one-dimensional case, similar facts were presented by E. Berkson and H. Porta in their study [14] of linear $C_{0}$-semigroups of composition operators on Hardy spaces. E. Vesentini investigated semigroups of those fractional-linear transformations which are isometries with respect to the infinitesimal hyperbolic metric on the unit ball of a Banach space (see [83-85]). He used them to study several important problems in the theory of linear operators on indefinite metric spaces. Note that, generally speaking, such semigroups are not everywhere differentiable in the infinite dimensional case. Since holomorphic self-mappings of a domain $D$ in a complex Banach space are nonexpansive with respect to any pseudometric $\varrho$ assigned to $D$ by a Schwarz-Pick system [35], it is natural to inquire whether mapping and fixed point theories analogous to the monotone and nonexpansive operator theories can be developed in the setting of these mappings.

In particular, it is of interest to study the asymptotic behavior of discrete and continuous semigroups of holomorphic mappings. This would, for example, provide information on the fixed (that is, stationary) points of the semigroups and therefore on the null points of their generators. In the one-dimensional case, the classical Denjoy-Wolff theorem provides information on both the location of fixed points and the behavior of the iterates 
of a holomorphic self-mapping. In recent years this theorem has been extended to higher dimensions in various ways. There are also analogues for continuous semigroups. See, for example, [1, 2, 14, 20, 52, 67, 69].

It turns out that the asymptotic behavior of solutions to evolution equations can be used to study the geometry of certain domains in complex spaces. For example, it is a well known result, due to $\mathrm{R}$. Nevanlinna [58], that if $f$ is holomorphic in the open unit disk $\{z \in \mathbb{C}:|z|<1\}$ and satisfies $f(0)=0, f^{\prime}(0) \neq 0$, then $f$ is univalent and maps the unit disk onto a star-like domain (with respect to 0 ) if and only if $\operatorname{Re}\left[z f^{\prime}(z) / f(z)\right]>0$ everywhere. This result, as well as most of the work on star-like functions on the unit disk, can be obtained from the identity

$$
\frac{\partial}{\partial \theta} \arg f\left(r e^{i \theta}\right)=\operatorname{Re}\left[\frac{r e^{i \theta} f^{\prime}\left(r e^{i \theta}\right)}{f\left(r e^{i \theta}\right)}\right] .
$$

This idea does not extend readily to a higher dimensional space. Moreover, such an approach is crucially dependent on the condition $f(0)=0$. Much later, Wald [86] gave a characterization of those functions which are star-like with respect to another center. Observe that although the classes of star-like, spiral-like and convex functions were studied very extensively, little was known about functions that are holomorphic on the unit disk $\Delta$ and star-like with respect to a boundary point. In fact, only in 1981 Robertson [73] introduced two relevant classes of univalent functions and conjectured that they are equal. In 1984 his conjecture was proved by Lyzzaik [53]. Finally, in 1990 Silverman and Silvia [75], using a similar method, gave a full description of the class of univalent functions on $\Delta$, the image of which is star-like with respect to a boundary point. However, the approaches used in all of these papers have a crucially one-dimensional character (because of the Riemann mapping theorem and Carathéodory's theorem on kernel convergence). In addition, the conditions given by Robertson and by Silverman and Silvia, characterizing star-likeness with respect to a boundary point, essentially differ from Wald's and Nevanlinna's conditions of star-likeness with respect to an interior point. Hence it is difficult to trace the connections between these two closely related geometric objects. Therefore, even in the one-dimensional case, the following problem arises: to find a unified condition of star-likeness (and spiral-likeness) with respect to either an interior or a boundary point. It seems that the idea to use a dynamical approach was first suggested by Robertson [72] and developed by Brickman [16], who introduced the concept of $\Phi$-like functions as a generalization of star-like and spiral-like functions (with respect to the origin) of a single complex variable. Suffridge [78, 80, 81], Pfaltzgraff [60-62] and Gurganus [34] developed a similar approach in order to characterize star-like, spiral-like (with respect to the origin), convex and closed-to-convex mappings in higher dimensional cases. Since 1970, the list of papers on these subjects is quite long. Nevertheless, it seems that there is no extension of Wald's as well as of the Silverman-Silvia results to higher dimensions.

Our main goal in this work is to study the asymptotic behavior of continuous holomorphic semigroups on domains in Hilbert and Banach spaces. We also combine analytic methods with the geometry of the domains in order to describe nonconservative evolution systems. 
More precisely, we study several problems concerning the following topics: flow invariance conditions on domains in complex spaces, characterizations of semicomplete and complete vector fields, asymptotic behavior of one-parameter flows, the null point set of a generator, applications to the theory of star-like and spiral-like mappings, and the solvability of certain classes of differential equations. For example, we observe that the above problems are, in turn, closely connected with the problem of the solvability of so-called generalized differential equations [64]. We show that the solutions to such equations can be expressed in terms of nonlinear semigroups.

The paper is organized as follows. The first part is devoted to basic concepts and definitions. We discuss, in particular, convex, star-shaped and spiral-shaped domains as well as fan-like and snail-like mappings. The second part is a brief historical sketch. Here we review star-like, spiral-like, convex and fan-shaped functions in the open unit disk, as well as their higher dimensional extensions. In the third and main part of the paper we present our dynamical approach to the study of biholomorphic mappings and of the geometry of domains in complex Banach spaces.

We begin with semicomplete vector fields and semigroups of holomorphic mappings, and continue with a discussion of stationary points of such semigroups and of boundary and interior flow invariance conditions. Of particular interest are the four examples presented in Section 3.3. We then characterize semicomplete vector fields on bounded symmetric domains. In Section 3.6 we introduce strongly semicomplete vector fields and establish rates of convergence for a class of semigroups generated by such vector fields. The next section, Section 3.7, is devoted to a new dynamical approach to the study of biholomorphic mappings and the connections between the asymptotic behavior of nonlinear semigroups and the geometry of domains in complex Banach spaces.

In Section 3.8 we are concerned with parametric representations of semicomplete vector fields on the Hilbert ball. We first present a characterization of semicomplete vector fields with an interior null point. We then study the asymptotic behavior of the semigroups generated by such vector fields. We introduce a notion of admissible upper and lower bounds, and use them to obtain rates of convergence in terms of both the norm and the hyperbolic metric. At the end of this section we establish a parametric representation of null point free semicomplete vector fields. We conclude the paper by presenting several new growth and covering theorems for star-like mappings defined on the open unit balls of Banach and Hilbert spaces.

\section{Basic concepts and definitions}

1.1. Holomorphic mappings. Let $X$ and $Y$ be two Banach spaces over the field of complex numbers $\mathbb{C}$, and let $D \subset X$ and $\Omega \subset Y$ be domains (open connected subsets) in $X$ and $Y$, respectively.

Definition 1.1.1. A mapping $f: D \rightarrow Y$ is called holomorphic if it is Fréchet differentiable at each point of $D$.

The set of all holomorphic mappings on $D \subset X$ with values in $\Omega \subset Y$ will be denoted by $\operatorname{Hol}(D, \Omega)$. In the special case when $X=Y$ and $D=\Omega$, the set $\operatorname{Hol}(D)=\operatorname{Hol}(D, D)$, 
consisting of all the holomorphic self-mappings of $D$, is a semigroup with respect to composition.

Definition 1.1.2. A mapping $f \in \operatorname{Hol}(D, \Omega)$ is said to be univalent on $D$ if for each pair of distinct points $x_{1}$ and $x_{2}$ in $D$ we have $f\left(x_{1}\right) \neq f\left(x_{2}\right)$.

In this case one can define the inverse mapping $f^{-1}: f(D) \rightarrow D$.

It is well known (see, for example, [39]) that if $X=\mathbb{C}^{n}$ and $Y=\mathbb{C}^{m}$ are finite dimensional complex spaces, then $f: D \subset \mathbb{C}^{n} \rightarrow \mathbb{C}^{m}, f \in \operatorname{Hol}\left(D, \mathbb{C}^{m}\right)$, is univalent if and only if $n=m$ and $f^{-1}$ is also holomorphic on $\Omega=f(D)$, i.e., $f^{-1} \in \operatorname{Hol}(\Omega, D)$. However, this fact is no longer true in the infinite dimensional case (see counterexamples in $[79,4])$. Therefore, in the general case a univalent mapping $f \in \operatorname{Hol}(D, \Omega), \Omega=f(D)$, is said to be biholomorphic if $f^{-1}: \Omega \rightarrow D$ belongs to $\operatorname{Hol}(\Omega, D)$. It is also known (see, for example, [29,49]) that if $f \in \operatorname{Hol}(D, \Omega)$ is biholomorphic, then for each point $x \in D$ the Fréchet derivative $A=f^{\prime}(x)$ is a linear isomorphism between $X$ and $Y$. In this situation we will say that $D$ and $\Omega$ are biholomorphically equivalent.

As we have already mentioned, in this case $X$ and $Y$ must be linearly isomorphic Banach spaces. Generally speaking, the converse is not true. That is, even if $X$ and $Y$ are isomorphic Banach spaces and $f \in \operatorname{Hol}(D, Y)$ has at each point $x$ in $D$ a continuously invertible Fréchet derivative, the mapping $f$ need not be univalent on $D$.

Nevertheless, in this case the mapping $f$ is biholomorphic on a neighborhood of each $x \in D$ by the inverse function theorem. In this situation we will say that $f \in \operatorname{Hol}(D, \Omega)$ is locally biholomorphic.

The set of all univalent mappings from a domain $D \subset X$ into $X$ will be denoted by $\operatorname{Univ}(D)$. For the special case when $D$ is the open unit ball of $X$, the subset of $\operatorname{Univ}(D)$ normalized by the conditions

$$
f(0)=0 \quad \text { and } \quad f^{\prime}(0)=I
$$

will be denoted by $S(D)$. This notation conforms to the one used in the classical onedimensional case, when $D=\Delta=\{z \in \mathbb{C}:|z|<1\}$. In this case we simply write

$$
S(=S(\Delta))=\left\{f \in \operatorname{Univ}(\Delta): f(0)=0 \text { and } f^{\prime}(0)=1\right\} .
$$

That is, $S$ consists of all the mappings $f \in \operatorname{Univ}(\Delta)$ such that $f$ has the following Taylor series at the origin:

$$
f(z)=z+\sum_{k=2}^{\infty} a_{k} z^{k} .
$$

\subsection{Convex, star-shaped and spiral-shaped domains}

Definition 1.2.1. A set $M$ in $X$ is called convex if for each pair of points $w_{1}$ and $w_{2}$ in $M$, the line segment joining $w_{1}$ and $w_{2}$ is contained in $M$, i.e., for each $t \in[0,1]$, the point $w=(1-t) w_{1}+t w_{2}$ is also in $M$.

If $D$ is a domain in $X$, then $f \in \operatorname{Univ}(D)$ is said to be a convex mapping on $D$ if its image $f(D)=\Omega$ is a convex domain in $X$. 
Definition 1.2.2. A set $M$ in $X$ is called star-shaped (with respect to the origin) if given any $w \in M$, the point $w_{t}=t w$ also belongs to $M$ for every $t$ with $0 \leq t \leq 1$. That is, if $M$ contains $w$, then it also contains the entire line segment joining $w$ to the origin.

Definition 1.2.3. If $D$ is a domain in $X$, then a biholomorphic mapping $f \in \operatorname{Hol}(D, X)$ is said to be a star-shaped mapping on $D$ if the closure $\bar{\Omega}$ of the image $\Omega=f(D)$ of $D$ is a star-shaped set (with respect to the origin).

In this definition the origin is in $\bar{\Omega}$. If, in particular, the origin belongs to $\Omega$, then we will say that $f$ is star-like, to make our definition consistent with the classical one. In this case the mapping $f$ has a null point $\tau$ in $D$. The set of all biholomorphic mappings on $D$ which are star-shaped on $D$ will be denoted by $\operatorname{Star}(D)$.

If, in addition, there is $\tau \in D$ such that

$$
f(\tau)=0,
$$

then we will write $f \in S_{\tau}^{*}(D)$. Of course, in this case such a point $\tau$ is unique because

$$
S_{\tau}^{*}(D) \subset \operatorname{Star}(D) \subset \operatorname{Univ}(D) .
$$

Again, in the one-dimensional case, when $X=\mathbb{C}$, we will simply write $S^{*}$ to denote the family of all biholomorphic (univalent) star-like functions $f$ on the unit disk $\Delta$ normalized by the conditions $f(0)=0$ and $f^{\prime}(0)=1$. That is,

$$
S^{*}=S \cap S_{0}^{*}(\Delta)
$$

In what follows the spectrum of a linear operator $A$ will be denoted by $\sigma(A)$.

Definition 1.2.4. A set $M$ in $X$ is said to be spiral-shaped (with respect to the origin) if there is a bounded linear operator $A: X \rightarrow X$ and a positive $\varepsilon$ such that $\operatorname{Re} \lambda \geq \varepsilon>0$ for all $\lambda \in \sigma(A)$ and such that for each $w \in M$ and $t \geq 0$, the point $e^{-t A} w$ also belongs to $M$.

Definition 1.2.5. If $D$ is a domain in $X$, then a biholomorphic mapping $f \in \operatorname{Hol}(D, X)$ is said to be a spiral-shaped mapping on $D$ if the closure $\bar{\Omega}$ of its image $\Omega=f(D)$ is a spiral-shaped set. Once again, if the origin belongs to $\Omega$, then, as in the classical case, we will say that $f$ is a spiral-like mapping on $D$.

The set of all biholomorphic mappings on $D$ which are spiral-shaped on $D$ will be denoted by $\operatorname{Spiral}(D)$. If, in addition, there is a point $\tau \in D$ such that $f(\tau)=0$, then we will write $f \in \operatorname{Sp}_{\tau}(D)$. Note also that if in Definition 1.2.4 the operator $A=I$, then $\Omega$ is actually star-shaped, i.e., $\operatorname{Star}(D) \subset \operatorname{Spiral}(D)$. Consequently, $S_{\tau}^{*}(D) \subset \operatorname{Sp}_{\tau}(D)$.

1.3. Fan-like and snail-like mappings. In both Definitions 1.2 .3 and 1.2 .5 the origin belongs to the closure of $\Omega$. Classical considerations usually cover the case when the origin belongs to $\Omega$. As far as we know, the situation when the origin belongs to $\partial \Omega$, the boundary of $\Omega$, was considered until now only in the one-dimensional case (see $[73,53,75]$ and the historical remarks below).

Definition 1.3.1. If $D$ is a domain in $X$, then a biholomorphic mapping $f \in \operatorname{Hol}(D, X)$ is said to be fan-like (respectively, snail-like) on $D$ if the closure $\bar{\Omega}$ of its image $\Omega=f(D)$ is 
a star-shaped (respectively, spiral-shaped) set and the origin belongs to $\partial \Omega$, the boundary of $\Omega$.

The set of all biholomorphic mappings on $D$ which are fan-like on $D$ will be denoted by $\operatorname{Fan}(D)$. The set of all snail-like mappings on $D$ will be denoted by $\operatorname{Snail}(D)$.

In other words, $f \in \operatorname{Fan}(D)$ if and only if $f$ belongs to $\operatorname{Star}(D)$ and has no null point in $D$. A mapping $f \in \operatorname{Snail}(D)$ if and only if $f \in \operatorname{Spiral}(D)$ and has no null point in $D$.

Sometimes it will be convenient to call a mapping $f \in \operatorname{Fan}(D)$ a star-like mapping with respect to a boundary point.

Finally, note that if $f \in \operatorname{Fan}(D)$ (or $f \in \operatorname{Snail}(D)$ ), then there is a point $e \in \partial D$ and a sequence $\left\{x_{n}\right\} \subset D$ such that $\left\{x_{n}\right\}$ strongly converges to $e$ and $\left\{f\left(x_{n}\right)\right\}$ strongly converges to zero.

\section{Historical sketch}

2.1. Star-like functions on the unit disk. The concept of univalent star-like functions was first introduced by Alexander [8] in 1915. In 1921 Nevanlinna [58] made a more detailed study of this class. In particular, the following characterization of the class $S^{*}=S \cap S_{0}^{*}(\Delta)$ is due to him.

Proposition 2.1.1. Let $f$ be a univalent holomorphic mapping on the unit disk $\Delta$ such that $f(0)=0$. Then $f$ is a star-like function on $\Delta$ if and only if

$$
\operatorname{Re}\left[\frac{z f^{\prime}(z)}{f(z)}\right]>0, \quad z \in \Delta .
$$

Intuitively, this result follows (as does most of the work on star-like functions on the unit disk) from the identity

$$
\frac{\partial}{\partial \theta} \arg f\left(r e^{i \theta}\right)=\operatorname{Re}\left\{\frac{r e^{i \theta} f^{\prime}\left(r e^{i \theta}\right)}{f\left(r e^{i \theta}\right)}\right\}
$$

which is valid whenever the function $f$ is holomorphic on $\Delta$ and not equal to zero at $z=r e^{i \theta}, r>0, \theta \in[0,2 \pi]$. Note also that if $f \in \operatorname{Hol}(\Delta, \mathbb{C})$ is locally biholomorphic, i.e., $f^{\prime}(z) \neq 0$ everywhere, and satisfies (2.1.1), then it is necessarily univalent.

Furthermore, condition (2.1.1) leads to the study of other interesting subclasses of $\operatorname{Univ}(\Delta)$. In particular, in 1936 Robertson [71] introduced the class $S^{*}(\lambda)$ of star-like functions of order $\lambda$ :

$$
S^{*}(\lambda)=\left\{f \in S^{*}: \operatorname{Re}\left[\frac{z f^{\prime}(z)}{f(z)}\right]>\lambda \geq 0, z \in \Delta\right\} .
$$

In 1978 Wald [86] characterized star-like functions with respect to another center. Using our notions, his result can be reformulated in the following way.

Proposition 2.1.2. Suppose that $f \in \operatorname{Hol}(\Delta, \mathbb{C})$ is either of the form

$$
f(z)=z+\sum_{k=2}^{\infty} a_{k} z^{k}
$$


or of the form $f(z)=\sum_{k=1}^{\infty} b_{k} z^{k}$ with $f^{\prime}(\tau)=1$ for some $\tau \in \Delta$. Then the function $g(z)=f(z)-f(\tau)$ belongs to $S_{\tau}^{*}(\Delta)$ if and only if $\operatorname{Re} q(z)>0$, where

$$
q(z)=\frac{(z-\tau)(1-z \bar{\tau}) f^{\prime}(z)}{f(z)-f(\tau)}=\frac{(z-\tau)(1-z \bar{\tau}) g^{\prime}(z)}{g(z)}, \quad z \neq \tau,
$$

and

$$
q(\tau)=1-|\tau|^{2}
$$

We will see below that condition (2.1.4) can easily be obtained by using another approach in more general settings. Different applications of (2.1.4) are presented in Wald's thesis [86] (see also [33]).

2.2. Spiral-like functions on the unit disk. It seems that the first occurrence of the class $\operatorname{Spiral}(\Delta)=\operatorname{Sp}_{0}(\Delta)$ arose when condition (2.1.1) was modified analytically by inserting the factor $e^{i \theta}$ :

$$
\operatorname{Re}\left[e^{i \theta} \frac{z f^{\prime}(z)}{f(z)}\right]>0, \quad z \in \Delta .
$$

(See Montel [57] and Špaček [76].) Actually, this definition is compatible with our Definition 1.2.5.

Proposition 2.2.1. Let $f \in \operatorname{Hol}(\Delta, \mathbb{C})$ have the form

$$
f(z)=z+\sum_{k=2}^{\infty} a_{k} z^{k}
$$

(i.e., $f(0)=0$ and $f^{\prime}(0)=1$ ). Then $f \in \operatorname{Sp}_{0}(\Delta)$ if and only if condition (2.2.1) holds for some $\theta \in(-\pi / 2, \pi / 2)$.

Note also that for a fixed $\theta \in(-\pi / 2, \pi / 2)$, a function $f$ satisfying (2.2.1) is called $\theta$-spiral-like.

2.3. Convex and close-to-convex functions. Historically, the notion of a convex function arose earlier than the notion of a star-like function. This seems quite natural, since convexity has played a crucial role in the development of analysis and geometry.

In 1913 Study [77] described univalent functions on a closed disk the image of which is a convex set. However, his condition employs the second derivative of the function. On the other hand, it is clear that a domain $\Omega$ is convex if and only if it is star-like with respect to each one of its points. Using this fact, Suffridge $[80,81]$ gave another characterization of convex functions which also holds for higher dimensions (see Section 2.5).

Here we quote another classical result due to Alexander [8] which provides an analytic connection between convex and star-like functions.

Proposition 2.3.1. Suppose that $f$ is a locally biholomorphic function on the disk $\Delta_{r}=$ $\{z:|z|<r\}$. Then $f$ is convex on $\Delta_{r}$ if and only if the function $g(z)=z f^{\prime}(z)$ is star-like on $\Delta_{r}$. 
As we mentioned above, in the study of the functions of the form

$$
f(z)=\sum_{k=1}^{\infty} a_{k} z^{k}
$$

that are holomorphic and univalent on the unit disk $\Delta$, certain subclasses the members of which share some simple geometric property arise rather naturally. Further developments in the classical theory have often had analytic generalizations and extensions. In 1952 Kaplan [43] defined the class of close-to-convex functions as those functions of the form (2.3.1) with $a_{1}=1$ such that

$$
\operatorname{Re}\left[\frac{f^{\prime}(z)}{\varphi^{\prime}(z)}\right]>0, \quad z \in \Delta,
$$

for some univalent convex function $\varphi$ on $\Delta$.

Since $\varphi$ is convex if and only if $h(z)=z \varphi^{\prime}(z)$ is star-like, (2.3.2) can be rewritten in the form

$$
\operatorname{Re}\left[\frac{z f^{\prime}(z)}{h(z)}\right]>0
$$

where $h$ is star-like on $\Delta$. If, in particular, $f$ is star-like itself, then we can take $h=f$ and (2.3.3) holds because

$$
\operatorname{Re}\left[\frac{z f^{\prime}(z)}{f(z)}\right]>0
$$

for $z \in \Delta$. Note also that condition (2.3.3) is often used as the definition of a close-toconvex function on $\Delta$, and that it is employed as a basic condition in higher dimensional generalizations (see Section 2.5).

2.4. Fan-shaped functions. A conjecture of M. S. Robertson. Observe that although classes of star-like functions and their applications have been studied very extensively, little is known about functions that are holomorphic on the unit disk $\Delta$ and star-like with respect to a boundary point. Only in 1981 Robertson [73] introduced two related classes of univalent functions and conjectured that they are equal. Following his notions and definitions we will say that a function $f$ belongs to the class $G^{*} \subset \operatorname{Fan}(\Delta)$ if $f$ is normalized by the following conditions:

(i) $f(0)=1$;

(ii) $f(1)=\lim _{r \rightarrow 1} f(r)=0, r \in[0,1)$;

(iii) for some real $\alpha, \operatorname{Re}\left[e^{i \alpha} f(z)\right]>0$;

(iv) the closure of $\Omega=f(\Delta)$ is star-shaped with respect to $f(1)=0$ (i.e., $\bar{\Omega}$ is a star-shaped set with respect to a boundary point);

(v) $f$ is either univalent on $\Delta$ or $f \equiv 1$.

Note that condition (iii) means that $\Omega$ lies in a half-plane of $\mathbb{C}$.

The other class introduced by Robertson has an analytic description. Let $G$ denote the class of holomorphic functions which do not vanish on $\Delta$, normalized by (i) and (ii), and such that for each $f \in G$,

$$
\operatorname{Re}\left\{\frac{2 z f^{\prime}(z)}{f(z)}+\frac{1+z}{1-z}\right\}>0, \quad z \in \Delta .
$$


Proposition 2.4.1 (Robertson [73]). $G \subset G^{*}$, that is, if $f$ satisfies (i), (ii), and (2.4.1), then $f \in G^{*}$.

Conjecture 2.4.2 (Robertson [73]). $G^{*} \subset G$.

In the same paper Robertson proved a slightly weaker result.

Proposition 2.4.3. If $f \in G^{*}$ has a holomorphic extension to a neighborhood of $\bar{\Delta}$, then $f \in G$.

Three years later Lyzzaik gave an affirmative answer to Robertson's conjecture.

Proposition 2.4.4 (Lyzzaik [53]). $G^{*} \subset G$, that is, Conjecture 2.4.2 is true.

Since condition (iii) is essential in their proofs, Propositions 2.4.1 and 2.4.4 cannot completely describe the class of fan-shaped functions on the unit disk.

More recently, Silverman and Silvia [75] showed that it is sufficient to change the coefficient 2 in (2.4.1) to describe the class of univalent functions the image of which is star-like with respect to a boundary point and lies in some angle of the plane with vertex at zero. More precisely, let $G_{\alpha}, 0 \leq \alpha<1$, denote the class of nonvanishing holomorphic functions on $\Delta$ which satisfy (i) and (ii), such that

$$
\operatorname{Re}\left\{\frac{z f^{\prime}(z)}{f(z)}+\frac{(1-\alpha)(1+z)}{1-z}\right\}>0, \quad z \in \Delta,
$$

and let $G_{\alpha}^{*} \subset \operatorname{Fan}(\Delta)$ denote the class of holomorphic functions $f$ on $\Delta$, satisfying conditions (i), (ii) and (v), such that the image $\Omega=f(\Delta)$ lies in a sector with aperture $2(1-\alpha) \pi$ and is star-shaped with respect to $f(1)=0$.

Proposition 2.4.5 ([75]). $G_{\alpha}^{*}=G_{\alpha}$ for each $\alpha \in[0,1)$.

However, the approaches used in these works have a crucially one-dimensional character (since they use one-dimensional phenomena such as the Riemann mapping theorem, the Bieberbach-de Branges theorem and Carathéodory's theorem on kernel convergence). Nevertheless, Robertson in his considerations showed that the class $G$ is contained in another class which turns out to be the key to the description of fan-shaped functions in higher dimensional cases.

Proposition 2.4.6 (Robertson [73]). Let $f \in G$ be nonconstant. Then the function $\log f, \log f(0)=0$, is univalent and close-to-convex in $\Delta$ with

$$
\operatorname{Re}\left\{(1-z)^{2} \frac{f^{\prime}(z)}{f(z)}\right\}<0, \quad z \in \Delta .
$$

As we will see below (Section 3.8.1), by using an entirely different approach, a more general form of condition (2.4.3) actually characterizes all fan-shaped functions on the unit disk. In other words, using our approach, we can show [28] that if $f$ is univalent and satisfies (2.4.3), then $f \in G_{\alpha}$ for some $0 \leq \alpha<1$. It will also be clear from the sequel that this condition can be generalized to the higher dimensional case.

2.5. Higher dimensional extensions. Until 1970 the literature on geometric properties of biholomorphic mappings in higher dimensional spaces $\left(\mathbb{C}^{n}\right.$, Hilbert spaces and 
Banach spaces) is rather limited (see [17, 55, 78]). Cartan [17] was the first mathematician who suggested the study of star-like and convex mappings in several complex variables despite the fact that many properties of univalent functions on the unit disk (e.g., the Riemann mapping theorem, Carathéodory's theorem on kernel convergence and the Bieberbach-de Branges theorem) fail in the higher dimensional case.

In 1970 Suffridge [78] established, inter alia, a necessary and sufficient condition for star-likeness which generalizes Proposition 2.1.1 to higher dimensions. He used the principle of subordination and one-dimensional ideas due to Robertson [72]. Furthermore, he also used a similar approach to describe star-like and spiral-like domains in Banach spaces (see [80, 81, 37, 32]).

Pfaltzgraff and Suffridge [62] gave a characterization of close-to-star-like holomorphic mappings which in the one-dimensional case coincides with a characterization of closeto-convex functions.

Roughly speaking, the idea in these considerations is the following one. If $f \in S$ (i.e., $f$ is a univalent holomorphic mapping on the unit disk $\Delta$ with $f(0)=0$ and $\left.f^{\prime}(0)=1\right)$, then the condition of star-likeness (2.1.1) can be rewritten in the form

$$
f(z)=f^{\prime}(z) g(z)
$$

where $g \in \operatorname{Hol}(\Delta, \mathbb{C})$ has the form

$$
g(z)=z p(z)
$$

with

$$
\operatorname{Re} p(z)>0, \quad z \in \Delta .
$$

Conditions (2.5.2) and (2.5.3) are equivalent to

$$
g(0)=0
$$

and

$$
\operatorname{Re} g(z) \bar{z}>0, \quad z \in \Delta, z \neq 0 .
$$

The latter condition can easily be generalized to the case of Hilbert and Banach spaces. Namely, let $X$ be a complex Banach space and let $X^{*}$ be the dual of $X$. By $\left\langle x, x^{*}\right\rangle$ we denote the action of a linear functional $x^{*}$ in $X^{*}$ on an element $x$ of $X$.

The mapping $J: X \rightarrow 2^{X^{*}}$ defined by

$$
J(x)=\left\{x^{*} \in X^{*}:\left\langle x, x^{*}\right\rangle=\|x\|^{2}=\left\|x^{*}\right\|^{2}\right\}, \quad x \in X,
$$

is called the (normalized) duality mapping.

Let $D$ be the open unit ball in $X$. We now define two families of holomorphic mappings on $D$. Let $N$ denote the subset of those mappings $g \in \operatorname{Hol}(D, X)$ which satisfy

$$
g(0)=0 \text { and } \operatorname{Re}\left\langle g(x), x^{*}\right\rangle>0
$$

for all $0 \neq x \in D$ and $x^{*} \in J(x)$.

Let

$$
M=\left\{g \in N: g^{\prime}(0)=I\right\},
$$

where $I$ denotes the identity operator on $X$. The following three assertions are due to Suffridge [80] and [81]. 
Proposition 2.5.1. Let $f$ be a locally biholomorphic mapping on $D$ (i.e., $f^{\prime}(x)$ is a bounded linear operator with a bounded inverse for each $x \in D)$ with $f(0)=0$. Then $f$ is star-like if and only if

$$
f(x)=f^{\prime}(x)[g(x)], \quad x \in D
$$

for some $g \in M$.

Note that condition (2.5.6) and the inclusion $g \in N$ imply the inclusion $g \in M$.

Proposition 2.5.2. Let $f$ be a locally biholomorphic mapping on $D$ and set

$$
f(x)-f(y)=f^{\prime}(x)[w(x, y)]
$$

for all $x, y \in D$. Then $f$ is a convex mapping on $D$ if and only if $\operatorname{Re}\left\langle w(x, y), x^{*}\right\rangle>0$ whenever $\|y\|<\|x\|$ and $x^{*} \in J(x)$.

The class $N$ is also useful in the characterization of spiral-like mappings.

Proposition 2.5.3. Let $A$ be a bounded linear operator which is strongly accretive, i.e., there is $\varepsilon>0$ such that

$$
\operatorname{Re}\left\langle A x, x^{*}\right\rangle \geq \varepsilon\|x\|^{2}
$$

for all $x \in X$ and $x^{*} \in J(x)$. Suppose that $f \in \operatorname{Hol}(D, X)$ is a locally biholomorphic mapping on $D$ which satisfies

$$
\begin{gathered}
f(0)=0, \\
f^{\prime}(0)=I .
\end{gathered}
$$

Then $f$ is spiral-like (relative to $A$ ) if and only if

$$
A f(x)=f^{\prime}(x)[g(x)]
$$

for some $g \in N$.

Remark 1. Actually, Proposition 2.5.1 follows from Proposition 2.5.3. Indeed, if $f$ is a locally biholomorphic mapping, then $f^{\prime}(0)=B$ is invertible. Setting $A=I$, one can consider the star-like mapping $\widetilde{f}=B^{-1} f$ which satisfies conditions (2.5.8) and (2.5.9) as well as equation (2.5.6) with $g \in N$. As we have mentioned above, $g$ must belong to $M$. Hence $f$ also satisfies (2.5.6) with the same $g$.

REMARK 2. In the above remark we used the auxiliary mapping $\widetilde{f}$ because of the normalizing condition (2.5.9) of Proposition 2.5.3. This condition is essential in the proof of the proposition used in $[80,81]$. The crucial point in that proof is that condition (2.5.9), $f^{\prime}(0)=I$, implies $g^{\prime}(0)=A$.

In fact, these conditions are needed because of the assumption (2.5.7) that $A$ is strongly accretive. This assumption was used by Suffridge [81] in his definition of spirallike mappings. Our Definition 1.2.5 is more general, since the spectrum of each strongly accretive operator lies strictly in the right half-plane. This enables us, in particular, to avoid the normalization (2.5.9) (see Section 3.7). Note also that in contrast with (2.5.7), our requirement of $A$ is independent of any equivalent norm on $X$. Since we are interested in the geometric properties of $\Omega$, our definition seems to be more natural. 
REMARK 3. It seems that for $\mathbb{C}^{n}$, Hilbert and Banach spaces the papers $[60,61]$ (see also [34]) were the first ones where the ideas of applying dynamical systems appeared. (In the one-dimensional case such ideas were employed earlier by Löwner, Kufarev [31, 33], Robertson [72] and Brickman [16].) Pfaltzgraff, for example, applied generalized Löwner differential equations to characterize subordination chains in $\mathbb{C}^{n}$ and univalent mappings on the unit ball. He also suggested this approach for Banach spaces.

The crucial point in their considerations is a connection of the classes $N$ and $M$ defined above with certain dynamical systems on $D$. Following Gurganus [34] we let $j(x) \in J(x)$ be such that $\langle y, j(\lambda x)\rangle=\bar{\lambda}\langle y, j(x)\rangle$ for all $\lambda \in \mathbb{C}$ and for all $y \in X$. Now we define the semi-inner product $[y, x]$ in $X$ by

$$
[y, x]=\langle y, j(x)\rangle
$$

This semi-inner product has the following properties:

$$
\begin{aligned}
& {[x+z, y]=[x, y]+[z, y],} \\
& {[\lambda y, x]=\lambda[y, x]} \\
& {[y, \lambda x]=\bar{\lambda}[y, x]} \\
& {[y, y]>0 \text { for } y \neq 0} \\
& |[y, x]|^{2} \leq[x, x][y, y] .
\end{aligned}
$$

Proposition 2.5.4. If $h \in N$, then for each $x \in D$ the initial value problem

$$
\left\{\begin{array}{l}
\frac{d v}{d t}+h(v)=0 \\
v(0)=x
\end{array}\right.
$$

has a unique solution $v=v(t, x)$ defined for all $t \geq 0$. For each $t \geq 0, v(t, \cdot)$ is a univalent Schwarz mapping on $D$, i.e., $v(t, \cdot) \in \operatorname{Hol}(D, D)$ and

$$
\|v(t, x)\| \leq\|x\|, \quad x \in D
$$

Moreover, if there is $k>0$ such that $\operatorname{Re}\left[h^{\prime}(0) x, x\right] \geq k\|x\|^{2}$, then

$$
\|v(t, x)\| \leq\|x\| \exp \left(-\frac{1-\|x\|}{1+\|x\|} k t\right) .
$$

In particular, if $h \in M$, then condition (2.5.15) holds with $k=1$.

Condition (2.5.15) immediately implies that for each $t \geq 0$, the origin is the unique fixed point of $v(t, \cdot): D \rightarrow D$, and that for each $x \in D, v(t, x)$ strongly converges to the origin as $t$ tends to infinity. In other words, the origin is an attractive fixed point for the family $\{v(t, \cdot)\}_{t \geq 0} \subset \operatorname{Hol}(D, D)$.

We will see below that, actually, the solvability of the Cauchy problem (2.5.13), the existence of a common fixed point for the solutions and its attractivity are the cornerstones of our dynamical approach to the study of the geometric properties of holomorphic mappings in a unified form. Moreover, we will see that such an approach is independent of the dimension of the space and of the domain on which the mapping is holomorphic. 


\section{Dynamical approach}

3.1. Semicomplete vector fields and semigroups of holomorphic mappings. We have already mentioned above that the dynamical approach to the study of the geometric properties of domains in a Banach space is based on an investigation of the solvability of the Cauchy problem

$$
\left\{\begin{array}{l}
\frac{d v}{d t}+g(v)=0 \\
v(0)=x
\end{array}\right.
$$

Definition 3.1.1. Let $D$ be a domain in $X$ and let $g \in \operatorname{Hol}(D, X)$. The mapping $g$ is said to be a semicomplete vector field on $D$ if the Cauchy problem (3.1.1) has a solution $\{v(t, x): t \geq 0\} \subset D$ which is well defined on $\mathbb{R}^{+}$for each initial value $x \in D$.

Note that since $g \in \operatorname{Hol}(D, X)$ is locally bounded (hence locally Lipschitzian), this solution is unique and the family $\{S(t)\}_{t \geq 0}, S(t):=v(t, \cdot)$, is a one-parameter semigroup (semiflow) of holomorphic self-mappings of $D$, i.e.,

$$
\left\{\begin{array}{l}
S(t+s)=S(t) \circ S(s) \text { for all } t, s \geq 0, \\
S(0)=I
\end{array}\right.
$$

where "o" denotes the composition operation in $\operatorname{Hol}(D)$ and $I$ is the restriction of the identity operator on $X$ to $D$. In the case where this semiflow consists of automorphisms of $D$, it can be extended to a one parameter group and the Cauchy problem (3.1.1) has a unique solution $\{v(t, x): t \in \mathbb{R}\} \subset D$ defined on all of $\mathbb{R}=(-\infty, \infty)$ for each initial value $x \in D$. The converse is also true. In such a situation the mapping $g$ is said to be a complete vector field (see [9, 23, 82]).

Complete vector fields also play a crucial role in the study of bounded symmetric domains in Banach spaces and their algebraic representations. We will see that semicomplete vector fields are a useful tool in the study of star-shaped and spiral-shaped domains. Returning to the notion of a semigroup, we observe that if the solution $S(t)=v(t, \cdot)$ of (3.1.1) is known, then $g \in \operatorname{Hol}(D, X)$ can be recovered as the strong limit

$$
g(x)=\lim _{t \rightarrow 0^{+}} \frac{x-S(t) x}{t},
$$

$(S(t) x:=v(t, x))$, i.e., $-g$ is the right derivative of the semigroup $S(t)$ at zero. In this case $g$ is said to be the infinitesimal generator of the semigroup.

As a matter of fact, for a hyperbolic domain the converse is also true: if for a given semigroup $\{S(t): t \geq 0\}$ of holomorphic self-mappings of $D$, which is continuous in $t \geq 0$, the strong limit $g(x)$ in (3.1.3) exists for all $x \in D$, then $v(t, x)=S(t) x$ is the solution of the Cauchy problem (3.1.1) for all $t \geq 0$ and $x \in D$. In other words, $g \in \operatorname{Hol}(D, X)$ is a generator of a semiflow on $D$ if and only if it is a semicomplete vector field (see, for example, [67]). 
Moreover, the limit (3.1.3) exists if and only if $S(t)$ converges to $I$ as $t \rightarrow 0^{+}$, uniformly on each subset $\widetilde{D}$ strictly inside $D$, i.e.,

$$
\lim _{t \rightarrow 0^{+}} \sup _{x \in \widetilde{D}}\|S(t) x-x\|=0 .
$$

In other words, a semigroup of holomorphic self-mappings of $D$ is right differentiable at $t=0^{+}$if and only if it is locally uniformly right continuous at $t=0^{+}$(see [14], [3], [70]). Note also that in this case $g \in \operatorname{Hol}(D, X)$ defined by (3.1.3) is bounded on each subset strictly inside $D$. Finally, we observe that if $\{G(t): t \in[0, a]\} \subset \operatorname{Hol}(D), a>0$, is an arbitrary family of holomorphic mappings such that the strong limit

$$
g(x)=\lim _{t \rightarrow 0^{+}} \frac{x-G(t) x}{t}
$$

exists locally uniformly on $D$ and $g$ is bounded on each subset strictly inside $D$, then $g$ is a semicomplete vector field on $D[70]$.

In addition, the solution $v(t, x)$ of the Cauchy problem (3.1.1) can be obtained by the product formula

$$
v(t, \cdot)=\lim _{t \rightarrow \infty}\left[G\left(\frac{t}{n}\right)\right]^{n},
$$

where the limit in (3.1.6) is taken with respect to the norm of $X$, uniformly on each subset strictly inside $D$. (Recall that $[F]^{n}$ denotes the $n$-fold iterate of a mapping $F$.)

A particular, but very important case is where such a family can be obtained by using the so-called nonlinear resolvents

$$
J_{r}=(I+r f)^{-1}, \quad r>0 .
$$

Definition 3.1.2. We will say that a mapping $f \in \operatorname{Hol}(D, X)$ satisfies the range condition $(R C)$ if for each $r>0$,

$$
(I+r f)(D) \supset D
$$

and the mapping $J_{r}$ in (3.1.7) is a well-defined holomorphic self-mapping of $D$.

Proposition 3.1.3 ([67]). Let $D$ be a bounded convex domain in $X$ and $f \in \operatorname{Hol}(D, X)$. Then $f$ is a semicomplete vector field on $D$ if and only if it satisfies the range condition $(R C)$. Moreover, in this case the solution $v(t, x), t \geq 0, x \in D$, of the Cauchy problem (3.1.1) can be obtained by the exponential formula

$$
v(t, x)=\lim _{n \rightarrow \infty}\left(I+\frac{t}{n} f\right)^{-n}(x),
$$

where the limit in (3.1.9) is uniform on each subset strictly inside D.

3.2. Stationary points of semigroups of holomorphic mappings. Let $D$ be a domain in $X$ and let $f$ be a semicomplete vector field on $D$. Suppose that $\{S(t): t \geq 0\}$ is the semigroup of holomorphic mappings generated by $f$. The uniqueness of the solution of the Cauchy problem (3.1.1) implies that the null point set of $f$ in $D$ coincides with the common fixed point set of $\{S(t)\}$, i.e.,

$$
\mathrm{Null}_{D} f=\bigcap_{t \geq 0} \operatorname{Fix}_{D} S(t) .
$$


In the theory of evolution equations this set is usually called the stationary point set of the semigroup. The topics in which we are interested here are: the structure of $\mathrm{Null}_{D} f$, existence and uniqueness theorems and the attractivity of a stationary point of $\{S(t)\}$ (that is, convergence theorems).

Definition 3.2.1. We will say that a point $a_{0} \in \operatorname{Null}_{D} f$ is quasi-regular if $A=f^{\prime}\left(a_{0}\right)$ satisfies the following condition:

$$
\operatorname{Ker} A \oplus \operatorname{Im} A=X .
$$

If, in particular, $A$ is an invertible linear operator (i.e., $\operatorname{Ker} A=\{0\}$ ), then we will say that $a_{0}$ is a regular point of $f$.

Proposition 3.2 .2 ([46]). Let $D$ be a bounded convex domain in $X$ and let $f$ be a semicomplete vector field on $D$ with a nonempty null point set $\mathrm{Null}_{D} f$. Suppose that one of the following hypotheses holds:

(i) $X$ is reflexive;

(ii) there is a quasi-regular null point $a_{0} \in \operatorname{Null}_{D} f$.

Then $\mathrm{Null}_{D} f$ is a holomorphic retract of D. Moreover, it is a complex analytic submanifold of $D$ which is tangent to $\operatorname{Ker} A=\operatorname{Ker} f^{\prime}\left(a_{0}\right)$. Consequently, if $a_{0}$ is a regular point of $\mathrm{Null}_{D} f$, then it is unique, i.e.,

$$
\mathrm{Null}_{D} f=\left\{a_{0}\right\} .
$$

A particular, but important case compatible with (i) is the case where $X=H$ is a complex Hilbert space. In this case $\mathrm{Null}_{D} f$ is an affine submanifold of $D$ which coincides with $\operatorname{Ker} A \oplus\left\{a_{0}\right\}$ (see $\left.[46,49,50]\right)$.

Definition 3.2.3 (see $[29,41]$ ). Let $f$ be a semicomplete vector field on a domain $D$ in $X$ with $\operatorname{Null}_{D} f \neq \emptyset$. A point $a_{0} \in \operatorname{Null}_{D} f$ is said to be locally uniformly attractive if the semigroup $\{S(t): t \geq 0\}$ generated by $f$ converges to $a_{0}$ in the topology of locally uniform convergence over $D$.

Proposition 3.2 .4 (cf. $[67,48]$ ). Let $D$ be a hyperbolic domain in $X$ and let $f$ be $a$ semicomplete vector field on $D$ with $\mathrm{Null}_{D} f \neq \emptyset$. Then

(1) for each $a_{0} \in \mathrm{Null}_{D} f$, the linear operator $A=f^{\prime}\left(a_{0}\right)$ generates the semigroup $\{B(t): t \geq 0\}=\left\{[S(t)]^{\prime}\left(a_{0}\right)\right\}$ of uniformly bounded linear operators, where $\{S(t): t \geq 0\}$ is the semigroup generated by $f$;

(2) for each $a_{0} \in \operatorname{Null}_{D} f$, the spectrum $\sigma(A)$ of the linear operator $A=f^{\prime}\left(a_{0}\right)$ lies in the right half-plane;

(3) if $f$ is bounded on each subset strictly inside $D$, then $a_{0}$ is an attractive point of $\{S(t)\}$ if and only if $\sigma(A)$ lies strictly inside the right half-plane, i.e., there is $\varepsilon>0$ such that for all $\lambda \in \sigma(A), \operatorname{Re} \lambda \geq \varepsilon>0$.

Note that in the latter case $a_{0}$ is, of course, a regular null point. In such a situation it is also called strictly regular.

3.3. Boundary and interior flow invariance conditions. Let $D$ be a convex subset of a Banach space $X$ and let $g: \bar{D} \rightarrow X$ be a continuous mapping on $\bar{D}$, the closure of $D$. 
Then the following tangency condition of flow invariance:

$$
\lim _{h \rightarrow 0^{+}} \operatorname{dist}(x-h g(x), \bar{D}) / h=0, \quad x \in \bar{D},
$$

is a necessary condition for the solvability of the evolution equation

$$
d v / d t+g(v)=0, \quad v(0)=x \in \bar{D} .
$$

This condition was systematically used to study the classes of monotone and accretive operators because of their connections with the theory of semigroups of nonexpansive mappings. For instance, a result of Martin [54] shows that if $D$ is a convex subset of $X$ and $g: \bar{D} \rightarrow X$ is a continuous accretive mapping on $\bar{D}$, then (3.3.1) is also sufficient for the existence of a solution of the Cauchy problem (3.1.1). Observe also that it was shown in [65] that in this case condition (3.3.1) is equivalent to

$$
\inf \operatorname{Re}\left\langle g(x), x^{*}\right\rangle \geq 0, \quad x \in \partial D,
$$

for each support functional $x^{*}$ of $\bar{D}$ at $x \in \partial D$ (that is, $x^{*} \in X^{*}$ and $\operatorname{Re}\left\langle x, x^{*}\right\rangle \geq \operatorname{Re}\left\langle y, x^{*}\right\rangle$ for all $y \in \bar{D})$.

For the class of holomorphic mappings an analog of Martin's theorem was established in $[67]$.

Proposition 3.3.1. Let $D$ be a bounded convex domain in $X$. If $g \in \operatorname{Hol}(D, X)$ has a uniformly continuous extension to $\bar{D}$, then it is semicomplete if and only if it satisfies the boundary flow invariance condition (3.3.1) (or (3.3.3)).

To illustrate some applications of this condition we consider a question regarding the solvability of autonomous differential equations of order $n$.

EXAmple 1 . Let $X$ be the $n$-dimensional complex space $\mathbb{C}=\left\{\left(z_{1}, \ldots, z_{n}\right): z_{j} \in \mathbb{C}\right\}$ with the $\ell_{p}$-norm

$$
\|z\|=\left(\sum_{k=1}^{n}\left|z_{k}\right|^{p}\right)^{1 / p}
$$

and let $D$ be the open unit ball in $X$. Suppose that $f: D \rightarrow \mathbb{C}$ is a holomorphic function on $D$ which has a continuous extension to $\bar{D}$. Defining $g=\left(g_{1}, \ldots, g_{n}\right): \bar{D} \rightarrow X$ in (3.3.2) by the formulae

$$
g_{i}\left(z_{1}, \ldots, z_{n}\right)= \begin{cases}-z_{i+1}, & 1 \leq i \leq n-1, \\ f\left(z_{1}, \ldots, z_{n}\right), & i=n,\end{cases}
$$

and using the standard method of rewriting an $n$th order differential equation as a first order system of $n$ equations, we deduce that the boundary condition

$$
\operatorname{Re} f(z) \bar{z}_{n} \geq\left|z_{n}\right|^{2-p} \operatorname{Re} \sum_{k=1}^{n-1} z_{k+1} \frac{\left|z_{k}\right|^{p}}{z_{k}}, \quad z \in \partial D,
$$

is fulfilled if and only if the equation

$$
x^{(n)}+f\left(x, x^{\prime}, \ldots, x^{(n-1)}\right)=0
$$

with the initial data

$$
x(0)=z_{1}, \quad x^{\prime}(0)=z_{2}, \ldots, x^{(n-1)}(0)=z_{n}
$$


has a unique solution $x=x\left(t, z_{1}, \ldots, z_{n}\right)$, defined for all $t \geq 0$ and $\left(z_{1}, \ldots, z_{n}\right) \in D$, which satisfies the estimate

$$
\|x(t)\|_{p, T}=\max _{0 \leq t \leq T}\left(|x(t)|^{p}+\left|x^{\prime}(t)\right|^{p}+\cdots+\left|x^{(n-1)}(t)\right|^{p}\right)^{1 / p}<1
$$

for each $T>0$.

EXAMPLE 2 (Liénard's Equation [10, 42]). Let us consider the second-order differential equation

$$
\ddot{x}+p(x) \dot{x}+q(x)=0
$$

with the initial data

$$
x(0)=z_{1}, \quad \dot{x}(0)=z_{2},
$$

where $p$ and $q$ are holomorphic functions on the closed unit disk of the complex plane, and

$$
\left|z_{1}\right|^{2}+\left|z_{2}\right|^{2}<1
$$

In this situation the boundary condition of Example 1 becomes

$$
\operatorname{Re}\left[p\left(z_{1}\right) \cdot z_{2}+q\left(z_{1}\right)-z_{1}\right] \bar{z}_{2} \geq 0 .
$$

It is easy to see that the latter inequality is equivalent to the conditions:

$$
\operatorname{Re} p(x) \geq 0, \quad|x|<1, \quad q(x)=x .
$$

ExAmple 3 (Van der Pol's Equation [10, 42]). The well-known equation describing nonharmonic oscillators

$$
\ddot{x}+\varepsilon\left(x^{2}-1\right) \dot{x}+x=0
$$

is a particular case of (LE). Hence, applying Example 2 we find that (VdPE) has a unique solution $x=x\left(t, z_{1}, z_{2}\right)$, defined for all $t \geq 0$ and $\left|z_{1}\right|^{2}+\left|z_{2}\right|^{2}<1$, which satisfies

$$
x(0)=z_{1}, \quad \dot{x}(0)=z_{2}, \quad|x(t)|^{2}+|\dot{x}(t)|^{2}<1, \quad t \geq 0,
$$

if and only if $\varepsilon \leq 0$.

EXAMPLE 4. The Lorenz equations

$$
\begin{aligned}
& \dot{x}=a(y-x), \\
& \dot{y}=x-y-x z, \\
& \dot{z}=x y-c z,
\end{aligned}
$$

arose in a model for convective motion in the atmosphere [42]. Here we show that for each pair of real parameters $(a, c)$, there is an initial point in the unit ball of $\mathbb{C}^{3}$ such that the solution of the system does not remain in the ball for all time. This is to be expected on physical grounds.

Indeed, setting

$$
g(x, y, z):=(a(x-y),-x+y+x z, c z-x y),
$$

we see that

$$
\operatorname{Re}\langle g(x, y, z),(x, y, z)\rangle=\operatorname{Re}\left\{(a \bar{x}-\bar{y})(x-y)+z \bar{y}(x-\bar{x})+c|z|^{2}\right\} .
$$


Now letting $x=y$ be purely imaginary and $z$ real, we obtain

$$
\operatorname{Re}\langle g(x, y, z),(x, y, z)\rangle=2 z|x|^{2}+c|z|^{2},
$$

which is negative when $x \neq 0, z<0$, and $|z|$ is small enough.

Thus boundary flow invariance conditions are seen to be quite useful. At the same time, in order to describe star-shaped and spiral-shaped domains, there is a need to find an interior flow invariance condition which will characterize the class of semicomplete vector fields. In addition, it does not seem natural to consider only boundary conditions because there are many examples of semicomplete vector fields defined on a domain $D$ which have no continuous extension to $\bar{D}$. Since we will mainly concentrate our discussions on domains which are biholomorphically equivalent to a ball, we will assume in what follows that $D$ is the open unit ball of a complex Banach space $X$. If $X=H$ is a Hilbert space with the inner product $\langle\cdot, \cdot\rangle$, then we will use the letter $\mathbb{B}$ to denote its open unit ball. This will enable us to point out special features of semicomplete vector fields in this case. Our approach to the search for different (but equivalent) characterizations of the class of semicomplete vector fields on $D$ is based on the following lemma.

Lemma 3.3.2. Let $D$ be the open unit ball in a complex Banach space $X$ and let $g \in$ $\operatorname{Hol}(D, X)$ satisfy

$$
\operatorname{Re}\left\langle g(x), x^{*}\right\rangle \geq \alpha(\|x\|)\|x\|
$$

for all $x \in D$ and some $x^{*} \in J(x)$, where $\alpha$ is a real continuous function on $[0,1)$ such that for all $\mu \in[0,1)$ and for all $r>0$ the equation

$$
s+\lambda \alpha(s)=\mu
$$

has a unique solution $s(\mu)$ in $[0,1)$. Then

(i) $g$ is a semicomplete vector field on $D$;

(ii) if $\beta(t, s)$ is the solution of the Cauchy problem

$$
\left\{\begin{array}{l}
\frac{\partial \beta(t, s)}{\partial t}+\alpha(\beta(t, s))=0 \\
\beta(0, s)=s \in[0,1)
\end{array}\right.
$$

and $v(t, x)$ is the solution of (3.1.1), then

$$
\|v(t, x)\| \leq \beta(t,\|x\|), \quad x \in D
$$

Proof. Fix $r \in[0,1)$ and $\lambda \geq 0$, and consider the equations

$$
\begin{aligned}
& x+\lambda g(x)=y, \\
& s+\lambda \alpha(s)=|y|,
\end{aligned}
$$

where $y \in D_{r}=\{x \in X:\|x\| \leq r<1\}$ and $s \in[0,1)$. It follows by our assumption that equation (3.3.9) has a unique solution $s_{0}=s_{0}(y) \in[0,1)$. Setting $\gamma(s)=s+\lambda \alpha(s)-|y|$ and $\delta>0$, we can find $\varepsilon>0$ such that $\gamma(s+\delta) \geq \varepsilon$. Taking $x \in D$ such that $\|x\|=s=s_{0}+\delta$, we have by (3.3.4) for such $x$ and some $x^{*} \in J(x)$,

$$
\operatorname{Re}\left\langle x+\lambda g(x)-y, x^{*}\right\rangle \geq s^{2}+\lambda \alpha(s) s-\|y\| s=s \gamma(s) \geq s \cdot \varepsilon .
$$


Now it follows by Theorem 3 in [7] that equation (3.3.8) has a unique solution $x=x(y)$ such that $\|x(y)\| \leq s_{0}+\delta$. Since $\delta$ is arbitrary, we must have

$$
\|x(y)\| \leq s_{0} .
$$

In terms of nonlinear resolvents the latter inequality can be rewritten as

$$
\left\|J_{\lambda}(y)\right\| \leq(1+\lambda \alpha)^{-1}\|y\| .
$$

Now we obtain our assertion by using Proposition 3.1.3 and the exponential formula given there.

\subsection{Bounded symmetric domains and complete vector fields}

Definition 3.4.1. A domain $D$ in $X$ is called symmetric if for each $a \in D$, there exists $F_{a} \in \operatorname{Aut}(D)$ such that $F_{a}^{2}=I_{D}$ and $a$ is an isolated fixed point of $F_{a}$.

For the case when $D$ is a bounded symmetric domain, the class aut $(D)$ of all complete vector fields on $D$ has been well described by using an algebraic approach (see, for example, [44, 82, 22, 9, 18]). By Kaup's theorem [44], every bounded symmetric domain can be realized as the open unit ball of a so-called $J B^{*}$-triple system. The simplest example of a bounded symmetric domain is the open unit disk $\Delta$ in the complex plane $\mathbb{C}$. In this case, if $g \in \operatorname{Hol}(\Delta, \mathbb{C})$ is a complete vector field (i.e., $g \in \operatorname{aut}(\Delta))$ which has a continuous extension to $\bar{\Delta}$, then the boundary flow invariance condition (3.1.2) implies that

$$
\operatorname{Re} g(z) \bar{z}=0, \quad z \in \partial \Delta .
$$

It is not difficult to check that a function $g$ satisfying (3.4.1) must be a polynomial of degree at most two, i.e.,

$$
g(z)=a+b z+c z^{2} .
$$

Moreover, the coefficients $a, b, c$ satisfy the relations

$$
\left\{\begin{array}{l}
a=-\bar{c}, \\
\operatorname{Re} b=0 .
\end{array}\right.
$$

So, $g$ is not only continuous on $\bar{\Delta}$; it is, in fact, holomorphic on all of $\mathbb{C}$. This also holds for the general case. The following assertion can be found in [82].

Proposition 3.4.2. Let $D$ be the open unit ball in a complex Banach space $X$. Then $\operatorname{aut}(D)$ is a real Banach Lie algebra and each $g \in \operatorname{aut}(D)$ is a polynomial of degree at most 2. Moreover, if

$$
P=\left\{g \in \operatorname{aut}(D): g^{\prime}(0)=0\right\}
$$

and $K=\{g \in \operatorname{aut}(D): g(0)=0\}$, then $\operatorname{aut}(D)=P \oplus K$.

Note that $K$ is actually the closed subalgebra of $\operatorname{aut}(D)$ consisting of the linear conservative operators $A: X \rightarrow X$, i.e., for all $x \in X$ and $x^{*} \in J(x)$,

$$
\operatorname{Re}\left\langle A x, x^{*}\right\rangle=0
$$

(see $[82,23])$. The set $P$ is the closed subspace consisting of the so-called "transvections":

$$
P=\left\{g \in \operatorname{aut}(D): g(x)=a-W_{a}(x)\right\},
$$


where $a$ is an element of $X$ and $W_{a}$ is a homogeneous form of the second order such that

$$
W_{i a}=-i W_{a} .
$$

In fact, the existence and uniqueness of transvections for each $a \in X$ is a necessary and sufficient condition for the unit ball of $X$ to be symmetric.

Proposition 3.4.3. Let $D$ be the open unit ball in a complex Banach space $X$. The following are equivalent:

(i) $D$ is a bounded symmetric domain in $X$;

(ii) for each $a \in X$, there is a unique two-homogeneous polynomial $W_{a}$ such that

$$
W_{i a}=-i W_{a}
$$

and the mapping $g \in \operatorname{Hol}(X, X)$ defined by

$$
g(x)=a-W_{a}(x)
$$

is a complete vector field on $D$;

(iii) $D$ is a homogeneous (or transitive) domain, i.e., for each pair $x$ and $y$ in $D$, there is $F \in \operatorname{Aut}(D)$ such that $F(x)=y$.

3.5. Characterizations of semicomplete vector fields. Returning to our main question concerning the formulation of an interior flow invariance condition for a semicomplete vector field, we first consider the case where the open unit ball $D$ of $X$ is a homogeneous (or bounded symmetric) domain. As we mentioned above, aut $(D)$ is a real Banach Lie algebra, while $\mathcal{G}(D)$ (the set of all semicomplete vector fields) is only a real cone. However, these facts give us immediately the following representation (see [47]).

Proposition 3.5.1. Let $X$ be a complex Banach space such that its open unit ball $D$ is homogeneous. Then the cone $\mathcal{G}$ of semicomplete vector fields on $D$ admits a decomposition

$$
\mathcal{G}=P \oplus N_{0},
$$

where $P$ is the real closed subspace of $\operatorname{Hol}(D)$ consisting of transvections and $N_{0}$ is the subcone of $\mathcal{G}$ such that for each $g \in N_{0}, g(0)=0$. In other words, $g \in \mathcal{G}$ admits a unique representation

$$
g=g_{1}+g_{2}
$$

where

$$
g_{1}(x)=g(0)-W_{p(0)}(x)
$$

is complete and $g_{2} \in \mathcal{G}$ is semicomplete with

$$
g_{2}(0)=0 .
$$

Note (see Proposition 3.5.2 below) that the cone $N_{0}$ can be described as follows:

$$
N_{0}=\left\{g \in \operatorname{Hol}(D, X): \operatorname{Re}\left\langle g(x), x^{*}\right\rangle \geq 0, \quad x \in D, x^{*} \in J(x)\right\} .
$$

(Compare this with the class $N$ in Section 2.5.) 
In the particular case where $X=\mathbb{C}$ is the complex plane and $D=\Delta$ is the open unit disk, (3.5.2) when combined with (3.5.3)-(3.5.5), becomes

$$
g(z)=g(0)-\overline{g(0)} z^{2}+z p(z)
$$

where $p(z) \in \operatorname{Hol}(\Delta, \mathbb{C})$ with

$$
\operatorname{Re} p(z) \geq 0, \quad z \in \Delta .
$$

That is, $p(z)$ is a function which belongs to the class of Carathéodory (see, for example, [33] and [25]).

As a consequence of Lemma 3.3.2, we also get that if $g \in \operatorname{Hol}(\Delta, \mathbb{C})$ has the representation (3.5.6) with (3.5.7), then it is a semicomplete vector field on $\Delta$.

Now by using the method of reduction to the one-dimensional case, one can characterize semicomplete vector fields in the general situation (cf. [6]).

Proposition 3.5.2. Let $D$ be the open unit ball in an arbitrary complex Banach space $X$, and let $g \in \operatorname{Hol}(D, X)$. Then

(I) $g$ is semicomplete on $D$ if and only if

(i) it is bounded on each subset strictly inside $D$;

(ii) $A=g^{\prime}(0)$ is an accretive linear operator on $X$, i.e.,

$$
\operatorname{Re}\left\langle A x, x^{*}\right\rangle \geq 0, \quad x \in X, x^{*} \in J(x),
$$

and

(iii) for each $x \in D$ and $x^{*} \in J(x)$,

$$
\begin{aligned}
\operatorname{Re}\left\langle\frac{1-\|x\|}{1+\|x\|} g^{\prime}(0) x+\left(1-\|x\|^{2}\right) g(0), x^{*}\right\rangle & \leq \operatorname{Re}\left\langle g(x), x^{*}\right\rangle \\
& \leq \operatorname{Re}\left\langle\frac{1+\|x\|}{1-\|x\|} g^{\prime}(0) x+\left(1-\|x\|^{2}\right) g(0), x^{*}\right\rangle .
\end{aligned}
$$

(II) If $\{S(t)\}_{t \geq 0}$ is the semigroup of holomorphic self-mappings of D generated by $g$, then

$$
\|S(t) x\| \leq \beta(t,\|x\|, a, m),
$$

where $m=\inf _{\|x\|=1} \operatorname{Re}\left\langle g(0), x^{*}\right\rangle, a=\inf _{\|x\|=1}\left\langle g^{\prime}(0) x, x^{*}\right\rangle$, and $\beta(t, s, a, m)$ satisfies the following algebraic equations:

$$
\begin{aligned}
\frac{\beta}{(1-\beta)^{2}} & =e^{-a t} \frac{s}{(1-s)^{2}} & & \text { if } m=0 ; \\
\frac{\beta}{(1-\beta)^{2}} & =-2 m t+\frac{1}{(1-s)^{2}} & & \text { if } a=-4 m \neq 0 ; \\
\frac{\beta^{2}+\beta(2+a / m)+1}{(1-\beta)^{2}} & =e^{-t(4 m+a)} \frac{s^{2}+s(2+a / m)+1}{(1-s)^{2}} & & \text { if } a \neq-4 m, m \neq 0 .
\end{aligned}
$$

Proof. (I) Sufficiency. If (i)-(iii) are fulfilled, then

$$
\operatorname{Re}\left\langle g(x), x^{*}\right\rangle \geq \operatorname{Re}\left\langle g(0), x^{*}\right\rangle\left(1-\|x\|^{2}\right)
$$

for all $x \in D$ and $x^{*} \in J(x)$. (Note that (3.5.9) is stronger than (3.5.10) because of (3.5.8).) But we already know (see Lemma 3.3.2) that (3.5.10) is sufficient for $g$ to be semicomplete. 
Necessity. Let now $g$ be a semicomplete vector field on $D$. Assertion (i) follows from Corollary 1 of [70].

To prove (ii) and (iii), let us fix any $x \in D$ and $x^{*} \in J(x)$, and set $u=x /\|x\|$ and $u^{*}=x^{*} /\|x\|$. Consider the holomorphic function $\widehat{g}$ on the unit disk defined as follows:

$$
\widehat{g}(\lambda)=\left\langle g(\lambda u), u^{*}\right\rangle, \quad \lambda \in \mathbb{C} .
$$

Similarly, we define a family $\{\widehat{S}(t)\}_{t \geq 0}$ of holomorphic self-mappings of $\Delta$ :

$$
\widehat{S}(t)(\lambda)=\left\langle S(t)(\lambda u), u^{*}\right\rangle, \quad \lambda \in \Delta, t \geq 0 .
$$

It is clear that

$$
\lim _{t \rightarrow 0^{+}} \frac{1}{t}(\lambda-\widehat{S}(t)(\lambda))=\widehat{g}(\lambda) .
$$

It follows from (3.1.5) and (3.1.6) that $\widehat{g}$ is a semicomplete vector field on $\Delta$. Hence, using (3.5.6), we get

$$
\widehat{g}(\lambda)=\widehat{g}(0)-\widehat{g}(0) \lambda^{2}+\lambda p(\lambda),
$$

where $p(\lambda) \in \operatorname{Hol}(\Delta, \mathbb{C})$ with

$$
\operatorname{Re} p(\lambda) \geq 0
$$

But it is well known that (3.5.14) implies that

$$
\operatorname{Re} p(0) \frac{1+|\lambda|}{1-|\lambda|} \geq \operatorname{Re} p(\lambda) \geq \operatorname{Re} p(0) \frac{1-|\lambda|}{1+|\lambda|}
$$

(see, for example, [31]). Noting that $p(0)=\widehat{g}^{\prime}(0)$, we get

$$
\operatorname{Re} \widehat{g}^{\prime}(0) \geq 0,
$$

and

$$
\operatorname{Re} \widehat{g}(\lambda) \bar{\lambda} \geq \operatorname{Re}\left[\widehat{g}^{\prime}(0)|\lambda|^{2} \frac{1-|\lambda|}{1+|\lambda|}+\operatorname{Re} \widehat{g}(0) \bar{\lambda}\left(1-|\lambda|^{2}\right)\right] \text {, }
$$

Setting in (3.5.16)-(3.5.18) $\lambda=\|x\|$, we get assertions (ii) and (iii).

(II) This estimate is a consequence of Lemma 3.3 .2 (see (3.3.7)).

REMARK 1. If $m=0$, then $\{S(t)\}_{t \geq 0}$ satisfies the explicit estimate

$$
\|S(t) x\| \leq e^{-a \frac{1-\|x\|}{1+\|x\|} t}\|x\|
$$

which gives a rate of convergence of $\{S(t)\}_{t \geq 0}$ to the origin, its stationary point.

REMARK 2. It can be shown that if in (3.5.8) equality holds for all $x \in X$ and $x^{*} \in J(x)$ (i.e., $A=g^{\prime}(0)$ is a conservative operator), then $g$ is actually a complete vector field.

REMARK 3. Proposition 3.5.2 and Lemma 3.3.2 also show that the simple inequality

$$
\operatorname{Re}\left\langle g(x), x^{*}\right\rangle \geq m\left(1-\|x\|^{2}\right)
$$

where $x^{*} \in J(x)$ and $m \leq 0$, is a necessary and sufficient condition for $g$ to be semicomplete. 


\subsection{Strongly semicomplete vector fields and rates of convergence of semi- groups}

Definition 3.6.1. Let $D$ be a domain in a Banach space $X$ and let $\mathcal{G}(D)$ be the family of all semicomplete vector fields on $D$. A mapping $g \in \mathcal{G}(D)$ is said to be a strongly semicomplete vector field if it has a unique null point in $D$ which is a locally uniformly attractive fixed point for the semigroup generated by $g$.

We have seen in Proposition 3.2.2 that if $D$ is a bounded domain, then $g \in \mathcal{G}(D)$ with $g(\tau)=0, \tau \in D$, is strongly semicomplete if and only if $\operatorname{Re} \sigma\left(g^{\prime}(\tau)\right) \geq \varepsilon>0$.

In this section we will give several sufficient conditions for $g$ to be strongly semicomplete on the open unit ball $D$ of $X$, and obtain rates of convergence for the semigroups generated by such mappings.

Recall that for a bounded convex domain $\Omega$ in $X$, all metrics assigned to it by a Schwarz-Pick system [35] coincide [24]. We call this unique metric the hyperbolic metric on $\Omega$.

Lemma 3.6.2. Let $D$ be the open unit ball in $X$ and let $g \in \operatorname{Hol}(D, X)$ satisfy the following condition:

$$
\operatorname{Re}\left\langle g(x), x^{*}\right\rangle \geq \alpha(\|x\|)\|x\|, \quad x \in D, x^{*} \in J(x),
$$

where $\alpha$ is a real continuous function on $[0,1]$ such that

$$
\alpha(1)=\omega>0 \text {. }
$$

Then

(i) $g$ is strongly semicomplete;

(ii) if $\{S(t)\}_{t \geq 0}$ is the semigroup generated by $g$, then for each pair $x$ and $y$ in $D$,

$$
\varrho(S(t) x, S(t) y) \leq e^{-\frac{\omega}{2} t} \varrho(x, y),
$$

where $\varrho$ is the hyperbolic metric on $D$. In particular, if $\tau \in D$ is the null point of $g$, then

$$
\varrho(S(t) x, \tau) \leq e^{-\frac{\omega}{2} t} \varrho(x, \tau)
$$

for all $x \in D$.

REMARK 1. Lemma 3.6.2 is different from Lemma 3.3.2 because we impose different conditions on the function $\alpha$. However, the proof of Lemma 3.6.2 is a modification of the proof of Lemma 3.3.2.

Proof of Lemma 3.6.2. Consider for each $n=1,2, \ldots$ the mappings $g_{n} \in \operatorname{Hol}(D, X)$ defined by

$$
g_{n}(x)=x+\frac{t}{n} g(x)-y, \quad x \in D,
$$

where $t \geq 0$ and $y \in D$. Let $D_{r}$ be the open ball centered at the origin of radius $r \in[0,1)$. For all $x \in \partial D_{r}=\{x \in X:\|x\|=r\}$ and for all $x^{*} \in J(x)$, we have by (3.6.1),

$$
\begin{aligned}
\operatorname{Re}\left\langle g_{n}(x), x^{*}\right\rangle & =\|x\|^{2}+\frac{t}{n} \operatorname{Re}\left\langle g(x), x^{*}\right\rangle-\operatorname{Re}\left\langle y, x^{*}\right\rangle \\
& \geq r^{2}+\frac{t}{n} r \alpha(r)-r\|y\|=r\left(r+\frac{t}{n} \alpha(r)-\|y\|\right) .
\end{aligned}
$$


Since $\alpha(1)>0$, it follows that for $n$ large enough the equation

$$
\varphi_{n}(r):=r+\frac{t}{n} \alpha(r)=1
$$

has a solution $r_{n} \in[0,1)$.

Indeed, $\varphi_{n}(0)=(t / n) \alpha(0) \leq 1$ for $n \geq t|\alpha(0)|$ and $\varphi_{n}(1)=1+(t / n) \omega>1$. The inequality (3.6.1) implies in turn that for such $n$ and $r_{n}$, and for all $x$ with $\|x\|=r_{n}$ and $x^{*} \in J(x)$,

$$
\operatorname{Re}\left\langle g_{n}(x), x^{*}\right\rangle \geq r_{n}(1-\|y\|) .
$$

Since $g_{n}$ is bounded on $\bar{D}_{r_{n}}$, it follows from [7] that the equation

$$
g_{n}(x)=x+\frac{t}{n} g(x)-y=0
$$

has a unique solution $x=J_{t / n}(y):=(I+t / n g)^{-1}(y) \in D_{r_{n}}$ for each $y \in D$. In other words, the resolvent mapping $J_{t / n}$ maps $D$ into $D_{r_{n}}$.

It now follows by the well-known Earle-Hamilton fixed point theorem [26] that $J_{t / n}$ has a unique fixed point $\tau$ in $D$. This point is also a null point of $g$. In addition, repeating the proof of the Earle-Hamilton theorem as presented in [30], we obtain the estimate

$$
\varrho\left(J_{t / n}(x), J_{t / n}(y)\right) \leq \frac{1}{1+(t / n)\left(\alpha\left(r_{n}\right) / 2\right)} \varrho(x, y)
$$

for each pair $x$ and $y$ in $D$.

Since $\alpha(r)$ is continuous on the interval [0,1], it follows by $(3.6 .7)$ that $r_{n} \rightarrow 1$ and $\alpha\left(r_{n}\right) \rightarrow \omega$ as $n \rightarrow \infty$. Therefore, by using the exponential formula

$$
S(t) x=\lim _{n \rightarrow \infty} J_{t / n}^{n}(x)
$$

and (3.6.9), we get by induction the estimates (3.6.3) and (3.6.4). Lemma 3.6.2 is proved. ExAmple 1. Let $D=\Delta$ be the open unit disk in the complex plane $\mathbb{C}$ and let $g \in$ $\operatorname{Hol}(\Delta, \mathbb{C})$ be defined by

$$
g(z)=a-\bar{a} z^{2}+b z \frac{1-c z}{1+c z}
$$

where $a \in \mathbb{C}, \operatorname{Re} b>0$ and $0 \leq c<1$. If we take

$$
\alpha(s)=-|a|\left(1-s^{2}\right)+(\operatorname{Re} b) s \frac{1-c s}{1+c s}
$$

then we get

$$
\operatorname{Re} g(z) \bar{z} \geq \alpha(|z|)|z|
$$

and $\alpha(1)=\operatorname{Re} b \frac{1-c}{1+c}>0$. Hence $g(z)$ is a strongly semicomplete vector field on $\Delta$.

EXAMPLE 2. In the theory of autonomous systems the following system is often considered:

$$
\left\{\begin{array}{l}
\dot{x}_{1}-x_{2}+x_{1} \varphi\left(x_{1}, x_{2}\right)=0 \\
\dot{x}_{2}+x_{1}+x_{2} \varphi\left(x_{1}, x_{2}\right)=0 .
\end{array}\right.
$$

We assume that the function $\varphi$ is holomorphic in the unit ball $\left|x_{1}\right|^{2}+\left|x_{2}\right|^{2}<1$. It is clear that for any point $x=\left(x_{1}, x_{2}\right) \in \mathbb{B}$, the support functional $x^{*}$ is defined by

$$
\left\langle y, x^{*}\right\rangle=y_{1} \bar{x}_{1}+y_{2} \bar{x}_{2} \text {. }
$$


Hence, for the mapping $g(x)=\left(-x_{2}+x_{1} \varphi(x), x_{1}+x_{2} \varphi(x)\right)$, we have

$$
\left\langle g(x), x^{*}\right\rangle=\left(\left|x_{1}\right|^{2}+\left|x_{2}\right|^{2}\right) \varphi(x) .
$$

Thus we have to examine three cases: either

1) there exists a point $x^{0}=\left(x_{1}^{0}, x_{2}^{0}\right) \in \mathbb{B}$ such that $\operatorname{Re} \varphi\left(x^{0}\right)<0$,

2) $\varphi(x)=i \beta, \beta \in \mathbb{R}$, or

3) $\operatorname{Re} \varphi(x)>0$.

In the first case the mapping $g$ is not a generator. In the third case $g$ is a strongly semicomplete vector field. In the second case $g$ is a group generator. The third case applies to the often used function $\varphi(x)=1+x_{1}^{2}+x_{2}^{2}$ (see, for example, [42, p. 327]). Thus the solution of the system

$$
\left\{\begin{array}{l}
\dot{x}_{1}-x_{2}+x_{1}\left(1+x_{1}^{2}+x_{2}^{2}\right)=0 \\
\dot{x}_{2}+x_{1}+x_{2}\left(1+x_{1}^{2}+x_{2}^{2}\right)=0
\end{array}\right.
$$

is well defined for all $t \geq 0$ and for all initial values in $\mathbb{B}$, and converges globally on $\mathbb{B}$ to the origin.

EXAMPLE 3. Now we will return to the differential equation from Example 1 in Section 3.3. It is clear that if we set

$$
\alpha(s):=\inf \left\{\Phi\left(x, x_{1}, \ldots, x_{n-1}\right):|x|^{p}+\left|x_{1}\right|^{p}+\cdots+\left|x_{n-1}\right|^{p}=s^{p}\right\},
$$

where

$$
\begin{aligned}
\Phi\left(x, x_{1}, x_{2}, \ldots, x_{n-1}\right)= & \operatorname{Re}\left[\frac{f\left(x, x_{1}, \ldots, x_{n-1}\right)\left|x_{n-1}\right|^{p}}{x_{n-1}}\right. \\
& \left.-\frac{x_{1}|x|^{p}}{x}-\frac{x_{2}\left|x_{1}\right|^{p}}{x_{1}}-\cdots-\frac{x_{n-1}\left|x_{n-2}\right|^{p}}{x_{n-2}}\right],
\end{aligned}
$$

then the inequality (3.6.1) holds. Consequently, if

$$
\liminf _{s \rightarrow 1^{-}}\left\{\Phi\left(x, x_{1}, \ldots, x_{n-1}\right):|x|^{p}+\cdots+\left|x_{n-1}\right|^{p}=s^{p}\right\}>0
$$

then by Lemma 3.6.2, the solution of the Cauchy problem in this example converges to the point $\left(x_{0}, 0, \ldots, 0\right)$ which is the unique null point of the generator $g$.

REMARK 2. Note that if $g \in \operatorname{Hol}(D, X)$ is known to be a semicomplete vector field on $D$, then condition (3.6.2) can be replaced by a slightly more general condition, namely,

$$
\alpha(l)>0 \quad \text { for some } l \in(0,1],
$$

which will still ensure assertion (i) of Lemma 3.6.2. This implies the following very simple and interesting sufficient condition.

Recall that a bounded operator $A: X \rightarrow X$ is said to be strongly accretive if

$$
\operatorname{Re}\left\langle A x, x^{*}\right\rangle \geq k\|x\|^{2}
$$

for some $k>0$ and all $x \in X, x^{*} \in J(x)$.

COROLlary 3.6.3. Let $g \in \mathcal{G}(D)$ and suppose that the linear operator $A=g^{\prime}(0)$ is strongly accretive, that is, it satisfies (3.6.10) for some $k>0$. If

$$
k>4\|g(0)\|,
$$

then $f$ is a strongly semicomplete vector field. 
Proof. Consider the function

$$
\alpha(s)=-\|g(0)\|\left(1-s^{2}\right)+k s \frac{1-s}{1+s} .
$$

Using (3.6.11), we see that $\alpha(1)=0$ and $\alpha^{\prime}(1)<0$. Hence there is $l \in(0,1)$ such that $\alpha(l)>0$. Since Proposition 3.5.2 shows that $\operatorname{Re}\left\langle g(x), x^{*}\right\rangle \geq \alpha(\|x\|)\|x\|$, the result follows by Remark 2 .

Note that if $A=g^{\prime}(0)$ is strongly accretive and $g(0)=0$, then condition (3.6.11) is fulfilled automatically. Hence the origin is an attractive fixed point of the semigroup generated by $g$. Actually, this fact also follows from more general considerations, and in this case one can obtain an exponential rate of convergence which will be useful in the sequel.

Proposition 3.6.4. Let $g \in \mathcal{G}(D)$ be such that $g(0)=0$ and $A=g^{\prime}(0)$ is strongly accretive with $\operatorname{Re}\left\langle A x, x^{*}\right\rangle \geq k\|x\|^{2}$. Suppose that $\{S(t)\}_{t \geq 0}$ is the semigroup generated by $g$. Then

$$
\begin{gathered}
\|S(t) x\| \leq\|x\| e^{-k \frac{1-\|x\|}{1+\|x\|} t}, \quad x \in D, t \geq 0 ; \\
\frac{\|S(t) x\|}{(1-\|S(t) x\|)^{2}} \leq e^{-k t} \frac{\|x\|}{(1-\|x\|)^{2}} .
\end{gathered}
$$

Proof. Both estimates follow directly from Lemma 3.3.2 if we set $\alpha(s)=k s(1-s) /(1+s)$. In this case $\beta(t, s) \leq s e^{-k \frac{1-s}{1+s} t}$, where $\{\beta(t, \cdot)\}_{t \geq 0}$ is the $\mathbb{R}$-valued semigroup generated by $\alpha$.

Estimate (i) is due to Gurganus [34, Proposition 2.5.4], while (ii) was obtained by Poreda [63]. Note that the condition $g(0)=0$ is essential in their considerations as well as in our approach above. In the case of a Hilbert space we will show below how more general estimates can be obtained when $g$ has an arbitrary null point which is strictly regular (see the note after Proposition 3.2.4).

\subsection{Flows and vector fields on biholomorphically equivalent domains and} geometric aspects in Banach spaces. The following simple assertion is the key to our subsequent considerations.

LEMMA 3.7.1. Let $D$ and $\Omega$ be two domains in a complex Banach space $X$ such that $\Omega=f(D)$ for some biholomorphic mapping $f: D \rightarrow \Omega$. Then there is a linear invertible operator $T$ from the space $\operatorname{Hol}(\Omega, X)$ onto the space $\operatorname{Hol}(D, X)$ which maps $\mathcal{G}(\Omega)$ onto $\mathcal{G}(D)$ (i.e., $\mathcal{G}(D)=T(\mathcal{G}(\Omega))$ ). In other words, the classes of semicomplete vector fields on $\Omega$ and $D$ are linearly isomorphic. Moreover, such an isomorphism $T: \mathcal{G}(\Omega) \rightarrow \mathcal{G}(D)$ can be given by the formulae

$$
\begin{aligned}
T(\varphi)(\cdot) & =\left[f^{\prime}(\cdot)\right]^{-1} \varphi(f(\cdot)) \\
T^{-1}(g)(\cdot) & =\left[f^{\prime}\left(f^{-1}(\cdot)\right)\right] g\left(f^{-1}(\cdot)\right),
\end{aligned}
$$

where $\varphi \in \mathcal{G}(\Omega)$ and $g \in \mathcal{G}(D)$. 
Proof. Let $\varphi \in \mathcal{G}(\Omega)$ and let $\left\{S_{\varphi}(t)\right\}_{t \geq 0}$ be the semigroup of holomorphic self-mappings on $\Omega$ generated by $\varphi$. Then it is clear that the family $\{G(t)\}_{t \geq 0}$ defined by

$$
G(t)=f^{-1} \circ S(t) \circ f
$$

is a semigroup of holomorphic self-mappings on $D$. Since the $X$-valued function $S(\cdot) x$ : $\mathbb{R}^{+} \rightarrow \Omega$ is differentiable for all $t \in[0, \infty)$, so is the function $G(\cdot) x: \mathbb{R}^{+} \rightarrow D$. Thus, for each $x \in D$ the strong limit

$$
g(x)=\lim _{t \rightarrow 0^{+}} \frac{1}{t}(x-G(t) x)
$$

exists and $g \in \mathcal{G}(D)$ is a semicomplete vector field on $D$ (see $\S 3.1$ ). In other words, for each $\varphi \in \mathcal{G}(\Omega)$, the mapping $g=T(\varphi)$ belongs to $\mathcal{G}(D)$, where $T: \operatorname{Hol}(\Omega, X) \rightarrow \operatorname{Hol}(D, X)$ is defined by (3.7.1).

It is clear that $T$ is an invertible linear operator and $T^{-1}$ is given by (3.7.2). Changing the roles of $\varphi$ and $g$, we see, by repeating the above considerations, that $T^{-1}$ takes $\mathcal{G}(D)$ onto $\mathcal{G}(\Omega)$. Lemma 3.7.1 is proved.

REMARK 1. It is clear that if $\varphi \in \operatorname{aut}(\Omega)$, then $g=T \varphi \in \operatorname{aut}(D)$ and conversely. If, in particular, $D=\Omega$ and $f \in \operatorname{Aut}(D)$, then the sets $\mathcal{G}(D)$ of all semicomplete vector fields and $\operatorname{aut}(D)$ of all complete vector fields are invariant under the operator $T: \operatorname{Hol}(D, X) \rightarrow$ $\operatorname{Hol}(D, X)$ defined by (3.7.1).

REMARK 2. Let $D$ and $\Omega$ be two domains in $X$ and let $f: D \rightarrow \Omega$ be a biholomorphism of $D$ onto $\Omega$. Define the operator $T: \operatorname{Hol}(\Omega, X) \rightarrow \operatorname{Hol}(D, X)$ by (3.7.1). If $\varphi \in \operatorname{Hol}(\Omega, X)$ has a null point $b$ in $\Omega$, then so does $g=T \varphi$ in $D$ and $a=f^{-1}(b)$ is a null point of $g$ in $D$.

In addition, by direct calculations we get

$$
g^{\prime}(a)=\left[f^{\prime}(a)\right]^{-1} \circ \varphi^{\prime}(b) \circ f^{\prime}(a)
$$

Thus, the linear operators $g^{\prime}(a): X \rightarrow X$ and $\varphi^{\prime}(b): X \rightarrow X$ are similar, hence they have the same spectrum. In particular, if $\varphi \in \mathcal{G}(\Omega)$ has a null point $b \in \Omega$, which is either quasi-regular, regular or strictly regular, then $a=f^{-1}(b)$ is also a quasi-regular, regular or strictly regular null point of $g$, respectively (see Section 3.2).

Using Lemma 3.7.1 and Remarks 1 and 2, we will subsequently present different parametric representations of semicomplete and complete vector fields. These representations will be useful, inter alia, in finding geometric characterizations of biholomorphic mappings in Hilbert spaces.

Now we will turn to a general description of spiral-shaped and star-shaped mappings defined on the unit ball in Banach spaces (see [28] for the one-dimensional case and compare [27] for a more general situation).

Proposition 3.7.2. Let $D$ be the open unit ball in a Banach space $X$ and let $f \in$ $\operatorname{Hol}(D, X)$ be a biholomorphic mapping on $D$. Then $f$ is spiral-shaped on $D$ if and only if there exist a linear operator $A$ such that its spectrum lies strictly inside the right half-plane and a real number $m, m \leq 0$, such that

$$
\operatorname{Re}\left\langle\left[f^{\prime}(z)\right]^{-1} A f(z), z^{*}\right\rangle \geq m\left(1-\|z\|^{2}\right)
$$


for all $z \in D$ and $z^{*} \in J(z)$. If $A=I$ in (3.7.6), then $f$ is actually star-shaped. In addition, if $f$ has a null point in $D$, then it is spiral-like (star-like); otherwise it is snail-like (fan-like).

Proof. If $f$ is spiral-shaped, then there exists a linear operator $A$ with its spectrum strictly in the right half-plane such that for each $y \in \Omega=f(D)$ and $t \geq 0$, the element $e^{-t A} y$ is in $\Omega$. This means that the vector field $\varphi=A$ is semicomplete on $\Omega$. Hence, by Lemma 3.7.1, the vector field

$$
g=T(A)=\left[f^{\prime}(\cdot)\right]^{-1} A f(\cdot)
$$

is semicomplete on $D$. Therefore (3.7.6) is a consequence of formula (3.5.19) (see Remark 3 in Section 3.5). Conversely, if (3.7.6) holds, then, again by Remark 3 of Section 3.5, the vector field $g$ defined by (3.7.7) is semicomplete on $D$. Therefore $A=T^{-1}(g)$ is semicomplete on $\Omega=f(D)$. That is, for each $y \in \Omega$, the curve $\left\{e^{-t A} y\right\}_{t \geq 0} \subset \Omega$ is the solution of the Cauchy problem

$$
\left\{\begin{array}{l}
\frac{d u}{d t}+A u=0 \\
u(0)=y
\end{array}\right.
$$

Now it follows by the assumption on $A$ and Definition 1.2.5 that $\Omega$ is spiral-shaped.

Note also that if $f$ has a null point $a \in D$, then so does $g$, and $f(a)=g(a)=0 \in \Omega$ is the limit point of $e^{-t A} y$ for each $y \in \Omega$. Hence in this case $f$ is spiral-like. Otherwise, that is, if $0 \in \partial \Omega$, the mapping $f$ is snail-like.

In addition, it is clear that if $A=I$, then the curve $\left\{e^{-t} y\right\}_{t \geq 0}$ is a straight line. In other words, $\Omega$ is star-shaped, and $f$ is either star-like or fan-like.

REMARK 3. As we saw in the proof of Proposition 3.7.2, in order to determine when $f \in \operatorname{Hol}(D, X)$ satisfying (3.7.6) is spiral-like (star-like) or snail-like (fan-like), we need to know whether $f$ has an interior null point. If it does not, then $f$ is snail-like (fan-like), and there is a sequence $\left\{x_{n}\right\} \subset D$ such that $x_{n} \rightarrow y \in \partial D$ and

$$
f\left(x_{n}\right) \rightarrow 0, \quad n \rightarrow \infty .
$$

Indeed, it is sufficient to set $x_{n}=f^{-1}\left(e^{-n A} z\right), n=0,1,2, \ldots$, for any $z \in f(D)$.

Generally speaking, the above question is equivalent to the existence of a null point of the semicomplete vector field $g$ defined by (3.7.7). Moreover, one can understand the condition of spiral-likeness (star-likeness) via the differential equation

$$
f^{\prime}(z) g(z)=A f(z)
$$

where $g$ is a strongly semicomplete vector field.

In addition, it turns out that if $f$ is a solution of (3.7.10) such that $f^{\prime}(z)$ is invertible for all $z \in D$, then $f$ is actually univalent, hence biholomorphic.

Indeed, since $g$ is strongly semicomplete, then by Definition 3.6.1, it has an interior null point $\tau \in D$, which is locally uniformly attractive for the semigroup $\left\{S_{g}(t)\right\}_{t \geq 0}$ generated by $g$. Furthermore, there is a neighborhood $U \subset D$ of the point $\tau$ such that $f$ is biholomorphic on $U$. Denote $V=f(U)$. Suppose by way of contradiction that $f$ is not univalent on $D$, that is, there are two distinct points $x_{1}$ and $x_{2}$ in $D$ such that 
$f\left(x_{1}\right)=f\left(x_{2}\right)=y \in f(D)$. But then $\left\{S_{g}(t) x_{1}\right\}$ and $\left\{S_{g}(t) x_{2}\right\}$ both converge to $\tau$ as $t$ tends to infinity, and therefore there is $t_{0} \geq 0$ such that both $S_{g}\left(t_{0}\right) x_{1}=u_{1}$ and $S_{g}\left(t_{0}\right) x_{2}=u_{2}$ belong to $U$.

Since $u(t)=e^{-t A}$ solves the Cauchy problem (3.7.8), the relation (3.7.3) becomes

$$
S_{g}\left(t_{0}\right)=f^{-1} \circ e^{-t A} \circ f .
$$

Hence $y_{i}=f\left(u_{i}\right)=e^{-t_{0} A} y \in V, i=1,2$. This implies that $y_{1}=y_{2}$ and consequently, $u_{1}=S_{g}\left(t_{0}\right) x_{1}=u_{2}=S_{g}\left(t_{0}\right) x_{2}$ which is impossible by the univalence of $S_{g}\left(t_{0}\right)$.

Finally, note that (3.7.10) and the chain rule also imply the equality $A=f^{\prime}(\tau) \circ$ $g^{\prime}(\tau) \circ\left[f^{\prime}(\tau)\right]^{-1}$, which means that the spectrum of $A$ lies strictly in the right half-plane. Thus we have proved the following assertion.

Proposition 3.7.3. Let $D$ be a domain in $X$ and let $f \in \operatorname{Hol}(D, X)$ be locally biholomorphic on $D$. Then $f$ is spiral-like if and only if there are a strongly semicomplete vector field $g$ on $D$ and a linear operator $A: X \rightarrow X$ such that $f$ satisfies the differential equation (3.7.10). If , in addition, $D$ is a hyperbolic domain endowed with a metric $\varrho$ which induces the norm topology, then there is a point $\tau \in D$ such that $f(\tau)=0$ and for each $\varrho$-ball $B_{r}$ centered at $\tau, B_{r}=\{x \in D: \varrho(x, \tau)<r\}$, the image $f\left(B_{r}\right)=\Omega_{r}$ is spiral-shaped (star-shaped, when $A=I$ ).

REMARK 4. The latter assertion follows from the fact that each $\varrho$-ball $B_{r}$ centered at $\tau$ is invariant under the semigroup $\{S(t)\}_{t \geq 0}$ generated by $g$. As a matter of fact, we will see below that in the above situation there is an equivalent norm of $X$ and a ball $B$ in this norm centered at $\tau$ such that $f(B)$ is spiral-shaped (star-shaped).

Corollary 3.7.4. Let $D$ be the open unit ball in $X$ and let $f \in \operatorname{Hol}(D, X)$ be a locally biholomorphic mapping on $D$ which satisfies the condition

$$
\operatorname{Re}\left\langle\left[f^{\prime}(z)\right]^{-1} A f(z), z^{*}\right\rangle \geq \alpha(\|z\|)\|z\|, \quad z \in D, z^{*} \in J(z),
$$

for some linear operator $A: X \rightarrow X$ and some real continuous function $\alpha:[0,1] \rightarrow \mathbb{R}$ such that $\alpha(1)>0$. Then $f$ is a univalent spiral-like (star-like) mapping on $D$.

REMARK 5. Condition (3.7.12) is a sufficient condition for $f$ to be spiral-like (or starlike), but it is not necessary. It would be nice, of course, to find a condition which is both necessary and sufficient for $f$ to be spiral-like (star-like). However, as already mentioned, to tackle this problem we need to find a condition which recognizes whether $f \in \operatorname{Hol}(D, X)$ (or equivalently, $g=\left[f^{\prime}(\cdot)\right]^{-1} A f(\cdot) \in \mathcal{G}(D)$ ) has an interior null point in $D$.

Generally speaking, these problems seem to be quite complicated. Moreover, there are examples of semicomplete vector fields on the closed unit balls $\bar{D}$ of Banach spaces which have no null point in $\bar{D}[45]$. Nevertheless, these problems can be solved completely in the case of the Hilbert ball. We will do it in the next section.

Recall that for a bounded linear operator $A: X \rightarrow X$ we denote by $\sigma(A)$ the spectrum of $A$. The number

$$
\kappa_{+}(A)=\max \{\operatorname{Re} \lambda: \lambda \in \sigma(A)\}
$$


is called the upper exponential index of $A$. It is well known (see [21]) that

$$
\kappa_{+}(A)=\lim _{t \rightarrow \infty} \frac{\log \left\|e^{t A}\right\|}{t}
$$

and for each $\omega>\kappa_{+}$there is a positive number $N=N(\omega)$ such that

$$
\left\|e^{t A}\right\| \leq N e^{\omega t}, \quad t \geq 0 .
$$

The number

$$
\kappa_{-}(A)=\min \{\operatorname{Re} \lambda: \lambda \in \sigma(A)\}=\lim _{t \rightarrow \infty} \frac{\log \left\|e^{-t A}\right\|}{-t}
$$

is called the lower exponential index of $A$.

Moreover, for each $\omega<\kappa_{-}$, there is a positive number $m=m(\omega)$ such that $\left\|e^{-t A}\right\| \leq$ $m e^{-\omega t}$.

If now $D$ is a bounded domain in $X$ and $g \in \mathcal{G}(D)$ has a null point $\tau \in D$, then in a neighborhood of $\tau$ the mapping $g$ can be represented by the Taylor series

$$
g(x)=A(x-\tau)+\sum_{k=l}^{\infty} P_{k}(x-\tau),
$$

where $A=g^{\prime}(\tau), l \geq 2$, and $P_{k}$ are homogeneous forms of order $k$. Thus $g$ is a strongly semicomplete vector field if and only if $\kappa_{-}(A)>0$.

Proposition 3.7.5. Let $D$ be a bounded domain in $X$ and let $g \in \mathcal{G}(D)$ admit the representation (3.7.13) in the neighborhood of $\tau \in D$. Suppose that $A=g^{\prime}(\tau)$ satisfies the condition

$$
0<\kappa_{+}(A)<l \kappa_{-}(A)
$$

Then for a given invertible operator $B \in L(X)$ such that $B A=A B$, the differential equation

$$
A f(x)=f^{\prime}(x) g(x)
$$

has a unique solution $f \in \operatorname{Hol}(D, X)$ which satisfies the initial conditions

$$
\left\{\begin{array}{r}
f(\tau)=0 \\
f^{\prime}(\tau)=B
\end{array}\right.
$$

If $\{S(t)\}_{t \geq 0}$ is the semigroup generated by $g$, then this solution can be represented by the formula

$$
f(x)=\lim _{t \rightarrow \infty} B e^{A t}(S(t) x-\tau),
$$

where the limit in (3.7.15) is taken with respect to the topology of locally uniform convergence over $D$.

Proof. We assume for simplicity that $\tau=0$.

Step 1. First we note that there is a norm $\|\cdot\|_{A}$ equivalent to the original norm of $X$ and a ball $\mathbf{B}$ in this norm centered at $\tau=0$ such that $g \in \mathcal{G}(\mathbf{B})$, i.e., $\mathbf{B}$ is invariant under the semigroup $\{S(t)\}_{t \geq 0}$ generated by $g$. Indeed, for a given $\mu \in\left(0, \kappa_{-}(A)\right)$, there is $m>0$ such that $\left\|e^{-t A}\right\| \leq m e^{-\mu t}$. Setting

$$
\|x\|_{A}=\sup _{t>0}\left\|e^{(\mu I-A) t} x\right\|,
$$


we get $\|x\| \leq\|x\|_{A} \leq m\|x\|$ and

$$
\left\|e^{-A s} x\right\|_{A} \leq e^{-\mu s}\|x\|_{A}, \quad s \geq 0 .
$$

The last inequality implies in turn that for each $x^{*} \in X^{*}$ such that $\operatorname{Re}\left\langle x, x^{*}\right\rangle=\|x\|_{A}^{2}=$ $\left\|x^{*}\right\|_{A}^{2}$ (i.e., $\left.x^{*} \in J_{A}(x)\right)$,

$$
\operatorname{Re}\left\langle A x, x^{*}\right\rangle \geq \mu\|x\|_{A}^{2}>0, \quad x \neq 0 .
$$

Now choose any ball $B_{r}=\left\{x \in X:\|x\|_{A}<r\right\} \subset D$ centered at the origin with radius $r>0$ and represent $g \in \mathcal{G}(D)$ by using the Taylor series (3.7.13) for $\tau=0$. We have

$$
g(x)=A x+\sum_{k=l}^{\infty} P_{k}(x), \quad l \geq 2,
$$

where $P_{k}$ are homogeneous polynomials of order $k \geq l$. If

$$
M=\sup _{\|x\| \leq r}\|g(x)-A x\|_{A},
$$

then it follows from the Schwarz Lemma that

$$
\|g(x)-A x\|_{A} \leq \frac{M}{r^{l}}\|x\|_{A}^{l}
$$

for all $x \in B_{r}$. Therefore we get

$$
\operatorname{Re}\left\langle g(x), x^{*}\right\rangle \geq \mu\|x\|_{A}^{2}-\frac{M}{r^{l}}\|x\|_{A}^{l+1}=\|x\|_{A}^{2}\left(\mu-\frac{M}{r^{l}}\|x\|_{A}^{l-1}\right)>0
$$

for all $x \in \mathbf{B}:=\left\{x \in X:\|x\|_{A}<\min \left\{\left(\mu r^{l} / M\right)^{1 /(l-1)}, r\right\}\right\}, x \neq 0$, and $x^{*} \in J_{A}(x)$. Hence $g$ is a semicomplete vector field on $\mathbf{B}$.

Step 2. Let now $h: \mathbf{B} \rightarrow X$ be any bounded holomorphic mapping on $\mathbf{B}$ such that

$$
h(0)=0, \quad D^{n} h(0)=0 \quad \text { for } 1 \leq n \leq l-1 .
$$

We claim that

$$
\lim _{t \rightarrow \infty} e^{t A} h(S(t) x)=0
$$

for all $x \in \mathbf{B}$. Indeed, without any loss of generality we may assume that the radius of $\mathbf{B}$ is 1. As above, (3.7.19) and the Schwarz Lemma imply that

$$
\|h(x)\|_{A} \leq M(h)\|x\|_{A}^{l}, \quad x \in \mathbf{B},
$$

where $M(h)=\sup _{x \in \mathbf{B}}\|h(x)\|_{A}$. In addition, it follows by Proposition 3.6.4 that for each $x \in \mathbf{B}$,

$$
\|S(t) x\|_{A} \leq e^{-\mu t} \frac{\|x\|_{A}}{\left(1-\|x\|_{A}\right)^{2}}\left(1-\|S(t) x\|_{A}\right)^{2} \leq e^{-\mu t} \frac{\|x\|_{A}}{\left(1-\|x\|_{A}\right)^{2}} .
$$

Now using (3.7.21), (3.7.22), and assumption (3.7.14) we can choose $\mu \in\left(0, \kappa_{-}\right)$and $\omega>\kappa_{+}$such that $\omega<l \mu$. Then we get

$$
\begin{aligned}
\left\|e^{t A} h(S(t) x)\right\|_{A} & \leq N(\omega) e^{\omega t}\|h(S(t) x)\|_{A} \leq N(\omega) \cdot M(h) e^{\omega t}\|S(t) x\|_{A}^{l} \\
& \leq N(\omega) M(h) e^{(\omega-l \mu) t} \frac{\|x\|^{l}}{(1-\|x\|)^{2 l}} \rightarrow 0
\end{aligned}
$$

as $t$ tends to infinity. The claim is proved. 
Step 3. Now we show that the limit in (3.7.15) exists for all $x \in D$. First, let us restrict ourselves to B. Consider the mapping $u: \mathbb{R}^{+} \times \mathbf{B} \rightarrow X$ defined as follows:

$$
u(t, x):=e^{A t} S(t) x, \quad t \geq 0, x \in \mathbf{B} .
$$

By calculations and the definition of $S(t)$, we get

$$
\frac{\partial u(t, z)}{\partial t}=A e^{A t} S(t) x-e^{A t} g(S(t) x)=-e^{A t} h(S(t) x)
$$

where $h(x)=g(x)-A x$ obviously satisfies (3.7.19). So we get from (3.7.25) and (3.7.23) that for each $x \in \mathbf{B}$ and $t_{1}, t_{2} \in \mathbb{R}^{+}$,

$$
\left\|u\left(t_{1}, x\right)-u\left(t_{2}, x\right)\right\|_{A} \leq N(\omega) M(h) \int_{t_{1}}^{t_{2}} e^{(\omega-l \mu) t} d t \cdot \frac{\|x\|^{2}}{(1-\|x\|)^{2 l}} .
$$

Thus the limit in (3.7.15) exists pointwise. Denote this limit by $f$. Let now $\widetilde{D}$ be any ball strictly inside $D$. It follows by (3.7.14) and Section 3.2 that $S(t) \rightarrow 0$ uniformly on $\widetilde{D}$. Hence there is a positive $p$ such that $y=S(p) x \in \mathbf{B}$ for each $x \in \widetilde{D}$. Furthermore, it follows by the semigroup property that for $x \in \widetilde{D}$,

$$
\begin{aligned}
\lim _{t \rightarrow \infty} B e^{A(p+t)} S(p+t) x & =\lim _{t \rightarrow \infty} B e^{A p} e^{A t} S(t) S(p) x \\
& =\lim _{t \rightarrow \infty} B e^{A p} e^{A t} S(t) y=e^{A p} f(y)=e^{A p} f(S(p) x) .
\end{aligned}
$$

This concludes Step 3.

Step 4. Next we will show the solvability of equation $(*)$. In fact, we claim that the mapping $f: D \rightarrow X$ defined by (3.7.15) is a solution of this equation with the initial data $f(0)=0$ and $f^{\prime}(0)=B$. Indeed, substituting $f=B \lim _{t \rightarrow \infty} e^{A t} S(t)$ in the right-hand side of $(*)$ we have by equation $(5.2)$ in [67],

$$
\begin{aligned}
f^{\prime}(x) g(x) & =B \lim _{t \rightarrow \infty} e^{t A}\left[\frac{\partial S(t) x}{\partial x} g(x)\right]=B \lim _{t \rightarrow \infty} e^{t A} g(S(t) x) \\
& =B \lim _{t \rightarrow \infty}\left[e^{t A} g^{\prime}(0) S(t) x+h(S(t) x)\right]
\end{aligned}
$$

where $h=g-A$. Again, by Step 2 we conclude that

$$
\lim _{t \rightarrow \infty} e^{A t} h(S(t) x)=0
$$

and

$$
f^{\prime}(x) g(x)=B A\left[\lim _{t \rightarrow \infty} e^{A t} S(t) x\right]=A f(x) .
$$

Step 5. Finally, it remains to show that if equation $(*)$ has another solution which satisfies the same initial conditions, then it must coincide with the mapping $f$ defined by (3.7.15). In fact, if $\tilde{f}: D \rightarrow X$ satisfies $(*), \widetilde{f}(0)=0$ and $\widetilde{f}^{\prime}(0)=B$, then we obtain

$$
A \widetilde{f}(S(t) x)=\widetilde{f}^{\prime}(S(t) x) g(S(t) x)=-\widetilde{f}^{\prime}(S(t) x) \frac{\partial S(t) x}{\partial t}
$$

for all $x \in D$ and $t \geq 0$. If we denote $G(t, x)=\tilde{f}(S(t) x), t \geq 0$, then this equality means that

$$
A G(t, x)=-\frac{\partial G(t, x)}{\partial t}
$$


In addition,

$$
G(0, x)=\widetilde{f}(x) .
$$

Therefore, solving (3.7.26) with the initial condition (3.7.27) we get $G(t, x)=e^{-t A} \widetilde{f}(x)$, or

$$
\widetilde{f}(x)=e^{t A} G(t, x)=e^{t A} \tilde{f}(S(t) s)=e^{t A}[B S(t) x+h(S(t) x)], \quad x \in D, t \geq 0,
$$

where $h=\widetilde{f}-B$. Letting $t$ tend to infinity and using Step 2, we get $\widetilde{f}(x)=f(x)$. The proposition is proved.

Corollary 3.7.6. Let $D$ be a bounded domain in $X$ and let $g \in \mathcal{G}(D)$ satisfy

$$
\begin{gathered}
g(\tau)=0, \\
g^{\prime}(\tau)=I
\end{gathered}
$$

for some $\tau \in D$. Then for a given invertible operator $B: X \rightarrow X$, the differential equation

$$
f(x)=f^{\prime}(x) g(x)
$$

has a unique solution which satisfies the initial conditions

$$
f(\tau)=0 \quad \text { and } \quad f^{\prime}(\tau)=B .
$$

If $\{S(t)\}_{t \geq 0}$ is the semigroup generated by $g$, then this solution can be represented as

$$
f=B \lim _{t \rightarrow \infty} e^{t}(S(t)-\tau) \text {. }
$$

Moreover, $f$ is biholomorphic on $D$ and $f(D)$ is a star-shaped domain.

Corollary 3.7.7. Let $D$ be a bounded domain in $X$ and let $f: D \rightarrow X$ be a star-like mapping on $D$ with respect to an interior point $\tau$ in $D$. Then there is a ball $B \subset D$ centered at $\tau$ such that $f(B)$ is star-shaped. Moreover, if $\varrho$ is a metric on $D$ assigned by a Schwarz-Pick system to D, then the image of each $\varrho$-ball B centered at $\tau$ is a star-shaped domain.

Proposition 3.7.8. A bounded domain $D$ in $X$ is biholomorphically equivalent to a starshaped domain if and only if there exists $g \in \mathcal{G}(D)$ such that for some $\tau \in D, g(\tau)=0$ and $g^{\prime}(\tau)=I$.

\subsection{Parametric representations of semicomplete vector fields on the Hilbert ball}

3.8.1. The one-dimensional Berkson-Porta formula. In 1978 E. Berkson and H. Porta [14] established a parametric representation of semicomplete vector fields on the unit disk $\Delta$ of the complex plane $\mathbb{C}$ :

A mapping $g: \Delta \rightarrow \mathbb{C}$ is a semicomplete vector field on $\Delta$ if and only if

$$
g(z)=(z-\tau)(1-z \bar{\tau}) p(z)
$$

for some $\tau \in \bar{\Delta}$ and $p(z) \in \operatorname{Hol}(\Delta, \mathbb{C})$ with $\operatorname{Re} p(z) \geq 0$ everywhere. This representation is unique.

If $\tau \in \Delta$, then it is the unique null point of $g$ (hence the stationary point of the semigroup $\{S(t)\}_{t \geq 0}$ generated by $g$ ). If $\tau \in \partial \Delta$, then $g$ has no null point in $\Delta$ and $\{S(t)\}_{t \geq 0}$ 
converges to $\tau$, uniformly on each subset strictly inside $\Delta$. Such a point $\tau$ is usually referred to as the Denjoy-Wolff point. Berkson and Porta used this representation to study eigenvalue problems for composition operators on Hardy spaces. Other applications of formula (3.8.1) can be found in [20].

Our motivation to generalize the Berkson-Porta representation to higher dimensions is the following observation regarding star-shaped mappings (star-like and fan-like) on $\Delta$. In fact, Proposition 3.7.3 and the representation (3.8.1) immediately imply the following fact: $A$ univalent mapping $f: \Delta \rightarrow \mathbb{C}$ is star-shaped on $\Delta$ if and only if for some $\tau \in \bar{\Delta}$,

$$
\operatorname{Re}\left\{\frac{(z-\tau)(1-z \bar{\tau}) f^{\prime}(z)}{f(z)}\right\} \geq 0 .
$$

Moreover, such a point $\tau$ is unique. If $\tau \in \Delta$, then $f$ is star-like (i.e., star-shaped with respect to an interior point) on $\Delta$; if $\tau \in \partial \Delta$, then $f$ is fan-like (i.e., star-shaped with respect to a boundary point). Note also that for $\tau=0$ we recover the classical result of Nevanlinna [58] (see Proposition 2.1.1) and for another $\tau \in \Delta$, (3.8.2) can be easily transformed into Wald's formula [86].

3.8.2. A characterization of semicomplete vector fields on the Hilbert ball with an interior null point. Here we consider a generalization of the Berkson-Porta formula for the case of the unit ball $\mathbb{B}$ in a complex Hilbert space $H$ with the inner product $\langle\cdot, \cdot\rangle$. For $y \in \mathbb{B}, y \neq 0$, we will denote by $M_{y}$ the following Möbius transformation of $\mathbb{B}$ :

$$
M_{y}(x)=\frac{1}{1+\langle x, y\rangle}\left(y+P_{y} x+s\left(I-P_{y}\right) x\right),
$$

where $I$ is the identity operator on $H, P_{y}=\left(\langle\cdot, y\rangle /\|y\|^{2}\right) y$ is the linear projection onto the one-dimensional subspace of $H$ spanned by $y$, and $s=\sqrt{1-\|y\|^{2}}$ (see, for example, [30]).

Note that

$$
\begin{gathered}
M_{-y}=\left[M_{y}\right]^{-1}, \\
M_{y}(0)=y, \quad M_{-y}(y)=0 .
\end{gathered}
$$

We also recall that the hyperbolic Poincaré metric $\varrho(\cdot, \cdot)$ on $\mathbb{B}$ can be given by the explicit formula

$$
\varrho(x, y)=\operatorname{arctanh}\left\|M_{-y}(x)\right\|=\operatorname{arctanh} \sqrt{1-\sigma(x, y)},
$$

where

$$
\sigma(x, y)=\frac{\left(1-\|x\|^{2}\right)\left(1-\|y\|^{2}\right)}{|1-\langle x, y\rangle|}=1-\left\|M_{-y}(x)\right\|^{2} .
$$

Note that $\varrho(x, y) \leq \varrho(u, v)$ if and only if $\sigma(x, y) \geq \sigma(u, v)$ and $x=y$ if and only if $\sigma(x, y)=1$.

For a point $\tau \in \overline{\mathbb{B}}$, the closure of $\mathbb{B}$, we also define the function $\varphi_{\tau}: \mathbb{B} \rightarrow \mathbb{R}^{+}$by

$$
\varphi_{\tau}(x)=\frac{|1-\langle x, \tau\rangle|^{2}}{1-\|x\|^{2}} .
$$

The sets

$$
E_{\tau}(k)=\left\{x \in \mathbb{B}: \varphi_{\tau}(x)<k\right\}, \quad k>1-\|\tau\|^{2},
$$


are "ellipsoids" in $\overline{\mathbb{B}}$ (see the details in [30]). If $\tau \in \partial \mathbb{B}$, the boundary of $\mathbb{B}$, then $E_{\tau}(k)$ are "ellipsoids" such that $\overline{E_{\tau}(k)} \cap \partial \mathbb{B}=\{\tau\}$. If $\tau \in \mathbb{B}$, then the sets $E_{\tau}(k)$ are strictly inside $\mathbb{B}$ and they are, in fact, open $\varrho$-balls centered at $\tau$ in the metric space $(\mathbb{B}, \varrho)$, i.e.,

$$
E_{\tau}(k)=\mathcal{B}(\tau, R)=\{x \in \mathbb{B}: \varrho(x, \tau)<R\}, \quad R=\operatorname{arctanh} \sqrt{1-\frac{1-\|\tau\|^{2}}{k}},
$$

(compare (3.8.6), (3.8.7) and (3.8.8)).

Finally, we observe that since $\mathcal{B}(\tau, R)$ can be rewritten in the form

$$
\mathcal{B}(\tau, R)=\left\{x \in B:\left\|M_{-\tau}(x)\right\| \leq \tanh R\right\},
$$

it follows that $M_{-\tau}$ maps the "ellipsoid" $E_{\tau}(k), k>1-\|\tau\|^{2}$, onto the open ball $\mathbb{B}_{r}=\{x \in \mathbb{B}:\|x\|<r\}$ centered at the origin, where $r=\tanh R<1$. This fact and Lemma 3.7.1 (see Section 3.7) allow us to characterize the class $N_{\tau}$ which consists of all the semicomplete vector fields on $\mathbb{B}$ which vanish at a given point $\tau \in \mathbb{B}$.

Indeed, let $g \in N_{\tau}, \tau \in \mathbb{B}$, and let $\{S(t)\}_{t \geq 0}$ be the semigroup of holomorphic selfmappings of $\mathbb{B}$ generated by $g$. Then for all $t \geq 0$ we have $S(t) \tau=\tau$, hence for all $x \in \mathbb{B}$,

$$
\varrho(S(t) x, \tau) \leq \varrho(x, \tau) .
$$

It follows from (3.8.9) that for each $k>1-\|\tau\|^{2}$ the "ellipsoid" $E_{\tau}(k)$ is $S(t)$-invariant for all $t \geq 0$. In other words, $g \in \mathcal{G}\left(E_{\tau}(k)\right)$, the class of all semicomplete vector fields on $E_{\tau}(k)$.

Set now in Lemma 3.7.1 $f=M_{\tau}$. Then $f$ maps biholomorphically the ball $\mathbb{B}_{r}=$ $\{x \in \mathbb{B}:\|x\|<r=\tanh R\}$ onto $E_{\tau}(k)$, where $R=\operatorname{arctanh} \sqrt{1-\left(1-\|\tau\|^{2}\right) / k}$. Consequently, the linear operator $T: \operatorname{Hol}(\mathbb{B}) \rightarrow \operatorname{Hol}(\mathbb{B})$ defined by

$$
T(g)(\cdot)=\left[\left(M_{\tau}\right)^{\prime}(\cdot)\right]^{-1} g\left(M_{\tau}(\cdot)\right)=\left(M_{-\tau}\right)^{\prime}\left(M_{\tau}(\cdot)\right) g\left(M_{\tau}(\cdot)\right)
$$

maps $\mathcal{G}\left(E_{\tau}(k)\right)$ onto $\mathcal{G}\left(\mathbb{B}_{r}\right)$ and $T(g)(0)=0$. We also note that since $M_{\tau}$ (as well as $M_{-\tau}$ ) are defined on all of $\mathbb{B}$, the operator $T$ is an isomorphism of $N_{\tau}$ onto $N_{0}$, the real cone of all semicomplete vector fields on $\mathbb{B}$ which vanish at the origin. But as we already know (see (3.5.5)), $N_{0}$ can be described as follows:

$$
N_{0}=\{h \in \operatorname{Hol}(\mathbb{B}, H): h(0)=0 \text { and } \operatorname{Re}\langle h(x), x\rangle \geq 0 \text { for all } x \in \mathbb{B}\} .
$$

Thus, setting $h=T(g)$ in (3.8.11) we get by direct calculations the following assertion.

Proposition 3.8.1. A mapping $g \in \operatorname{Hol}(\mathbb{B}, H)$ belongs to

$$
N_{\tau}=\{g \in \mathcal{G}(\mathbb{B}): g(\tau)=0\},
$$

for some $\tau \in \mathbb{B}$ if and only if

$$
\operatorname{Re} \frac{\langle g(x), x\rangle}{1-\|x\|^{2}} \geq \operatorname{Re} \frac{\langle g(x), \tau\rangle}{1-\langle x, \tau\rangle}, \quad x \in \mathbb{B} .
$$

Alternative proof. It was shown in $[68]$ that $g \in \mathcal{G}(\mathbb{B})$ if and only if it is $\varrho$-monotone, i.e., for each pair $x, y \in \mathbb{B}$ and $r \geq 0$,

$$
\varrho(x+r g(x), y+r g(y)) \geq \varrho(x, y)
$$

whenever $x+r g(x)$ and $y+r g(y)$ belong to $\mathbb{B}$. Also, it was proved there that (3.8.14) 
can be written in an equivalent form:

$$
\operatorname{Re}\left[\frac{\langle g(x), x\rangle}{1-\|x\|^{2}}+\frac{\langle g(y), y\rangle}{1-\|y\|^{2}}\right] \geq \operatorname{Re} \frac{\langle g(x), y\rangle+\langle g(y), x\rangle}{1-\langle x, y\rangle} .
$$

If now $g(\tau)=0$ for some $\tau \in \mathbb{B}$, then setting $y=\tau$ in (3.8.15) we get (3.8.13).

Conversely, let (3.8.13) hold for some $\tau \in \mathbb{B}$. Define

$$
x^{\dagger}=\frac{x}{1-\|x\|^{2}}-\frac{\tau}{1-\langle\tau, x\rangle} .
$$

Then (3.8.13) can be rewritten as

$$
\operatorname{Re}\left\langle g(x), x^{\dagger}\right\rangle \geq 0, \quad x \in \mathbb{B} .
$$

Now it can be shown that for each $k>1-\|\tau\|^{2}$ and for each pair $x \in \partial E_{\tau}(k)$ and $y \in \overline{E_{\tau}(k)}$,

$$
\operatorname{Re}\left\langle x, x^{\dagger}\right\rangle \geq \operatorname{Re}\left\langle y, x^{\dagger}\right\rangle
$$

That is, $x^{\dagger}$ is a support functional of the convex set $\overline{E_{\tau}(k)}$ at the point $x$. Now applying Proposition 3.3.1 we get by (3.8.17) that $g$ belongs to $\mathcal{G}\left(E_{\tau}(k)\right)$. Letting $k$ tend to infinity we also see that $g \in \mathcal{G}(\mathbb{B})$, because $\bigcup_{k>1-\|\tau\|^{2}} E_{\tau}(k)=\mathbb{B}$. This concludes the proof.

3.8.3. Comparison of solutions of evolution equations: asymptotic behavior. For the one-dimensional case, formula (3.8.13) is equivalent to the Berkson-Porta representation (3.8.1) when $g \in \mathcal{G}(\Delta)$ with a null point $\tau \in \Delta$. To get an analog of (3.8.1) for $g \in \mathcal{G}(\mathbb{B})$ with no null point in $\mathbb{B}$, we need another approach, because there is no Möbius transformation which translates a ball centered at the origin onto an ellipsoid internally tangent to the boundary of $\mathbb{B}$. An additional important problem which arises even when $\tau \in \mathbb{B}$, is to establish a characterization of the cone $\mathcal{G}=\mathcal{G}(\mathbb{B})$ and its subcones $N_{\tau}$ which could show when $g \in \mathcal{G}$ is a strongly semicomplete vector field.

Our purpose is first to study the latter problem. Moreover, we will also establish a one-to-one correspondence between our characterization of strongly semicomplete vector fields and the rates of convergence of their generated flows.

Let $\alpha:[0,1] \rightarrow \mathbb{R}$ be a continuous function on the interval $[0,1]$ such that for some $\delta>0$ and for each $r \in[0, \delta)$, the function $s+r \alpha(s)$ is increasing on $[0,1]$. We assume also that $\alpha$ satisfies the following range condition: for each $r \in[0, \delta)$ and for all $p \in[0,1]$, the equation

$$
s+r \alpha(s)=p
$$

has a (unique) solution $s=s(r, p) \in[0,1]$. As we have already mentioned, this solution is an increasing function of $p \in[0,1]$ for each fixed $r \in[0, \delta)$. Also, for each $t \geq 0$, the limit

$$
\beta(t, p)=\lim _{n \rightarrow \infty} s^{[n]}\left(\frac{1}{n} t, p\right)
$$

exists, where by $s^{[n]}(r, p)$ we denote the $n$-fold iterate of $s(r, \cdot)$, i.e., $s^{[0]}(r, p)=p$, $s^{[n]}(r, p)=s^{[n-1]}(r, s(r, p)), n=1,2, \ldots$ This limit is the solution of the Cauchy problem

$$
\left\{\begin{array}{l}
\frac{\partial \beta(t, p)}{\partial t}+\alpha(\beta(t, p))=0 \\
\beta(0, p)=p
\end{array}\right.
$$


Let now $g$ be a holomorphic mapping on $\mathbb{B}$, the open unit ball of the Hilbert space $H$. Since $g$ is continuously differentiable, hence locally Lipschitz, the Cauchy problem

$$
\left\{\begin{array}{l}
\frac{\partial u(t, x)}{\partial t}+g(u(t, x))=0 \\
u(0, x)=x
\end{array}\right.
$$

has a unique local solution $u=u(t, x)$ which is real-analytic in $t$ in some neighborhood of zero, and holomorphic in a neighborhood $U_{x}$ of $x$. We intend to compare this solution with the function $\beta(t, p)$ defined by (3.8.20) and to recognize when $u=u(t, x)$ can be extended to a global solution of (3.8.21) defined on $\mathbb{R}^{+} \times \mathbb{B}$. To do this for a given $\tau \in \mathbb{B}$, we define a function $m_{\tau}:[0, \delta) \times U_{x} \rightarrow \mathbb{R}^{+}$by

$$
m_{\tau}(t, x)=\left\|M_{-\tau}(u(t, x))\right\|,
$$

where $M_{-\tau}$ is the Möbius transformation which takes $\tau$ to zero. It is natural to compare this function with the function $\beta\left(t,\left\|M_{-\tau}(x)\right\|\right)$. Moreover, we will see below that local and global relations between these functions are completely determined by their derivatives at $t=0^{+}$.

Proposition 3.8.2. Let $g \in \operatorname{Hol}(\mathbb{B}, H)$ and let $\alpha, \beta$, u and $m_{\tau}$ be the functions defined above. Then the following assertions are equivalent:

(i) for some $\tau \in \mathbb{B}$,

$$
m_{\tau}(t, x) \leq \beta\left(t,\left\|M_{-\tau}(x)\right\|\right)
$$

whenever $u(t, x)$ is defined;

(ii) for some $\tau \in \mathbb{B}$,

$$
\left.\frac{\partial m_{\tau}}{\partial t}\right|_{t=0^{+}} \leq\left.\frac{\partial \beta}{\partial t}\right|_{t=0^{+}}=\alpha\left(\left\|M_{-\tau}(x)\right\|\right) ;
$$

(iii) for some $\tau \in \mathbb{B}$, the mapping $g$ belongs to $N_{\tau}$ and

$$
\operatorname{Re}\left\langle g(x), x^{\dagger}\right\rangle \geq \frac{\alpha\left(\left\|M_{-\tau}(x)\right\|\right)\left\|M_{-\tau}(x)\right\|}{\sigma(\tau, x)},
$$

where $\sigma(x, y)=1-\left\|M_{-y}(x)\right\|^{2}$ and $x^{\dagger}=x /\left(1-\|x\|^{2}\right)-\tau /(1-\langle\tau, x\rangle)($ see (3.8.7) and (3.8.16)).

When these assertions hold, $u(t, x)$ has a unique extension to all of $\mathbb{R}^{+} \times \mathbb{B}$ and the estimate (i) is true globally. The point $\tau$ in (i)-(iii) is one and the same.

Proof. Note that $m_{\tau}(0, x)=\beta\left(0,\left\|M_{-\tau}(x)\right\|\right)$, so (i) $\Rightarrow$ (ii) is trivial. Furthermore, by direct calculations we obtain

$$
\left.\frac{\partial m_{\tau}}{\partial t}\right|_{t=0^{+}}=-\frac{\sigma(\tau, x)}{\left\|M_{-\tau}(x)\right\|} \operatorname{Re}\left\langle g(x), x^{\dagger}\right\rangle .
$$

This concludes the proof of the implication (ii) $\Rightarrow$ (iii) because

$$
\left.\frac{\partial \beta\left(t,\left\|M_{-\tau}(x)\right\|\right)}{\partial t}\right|_{t=0^{+}}=-\alpha\left(\left\|M_{-\tau}(x)\right\|\right) .
$$

Thus it remains to be shown that (iii) $\Rightarrow(\mathrm{i})$. To this end, we again use Lemma 3.7 .1 of Section 3.7. First, we note that (iii) and Proposition 3.8.1 imply that $g \in N_{\tau}$, hence 
$u(t, x)$ is well defined and belongs to $\mathbb{B}$ for all $(t, x) \in \mathbb{R}^{+} \times \mathbb{B}$. Therefore the operator $T: \operatorname{Hol}(\mathbb{B}) \rightarrow \operatorname{Hol}(\mathbb{B})$ defined by (3.8.11) takes $g \in N_{\tau}$ to $\varphi \in N_{0}$. Since the explicit expression for the linear operator $A(=A(x)):=M_{-\tau}^{\prime}\left(M_{\tau}(x)\right)$ is

$$
A=\frac{1+\langle x, \tau\rangle}{1-\|\tau\|^{2}}\left(P_{\tau}+\sqrt{1-\|\tau\|^{2}}\left(I-P_{\tau}\right)+x\langle\cdot, \tau\rangle\right),
$$

we get

$$
\begin{aligned}
\langle\varphi(x), x\rangle & =\left\langle A g\left(M_{\tau}(x)\right), x\right\rangle\left\langle g\left(M_{\tau}(x)\right), A^{*} x\right\rangle \\
& =\frac{|1+\langle x, \tau\rangle|^{2}}{1-\|\tau\|^{2}}\left\langle g\left(M_{\tau}(x)\right), M_{\tau}(x)-\tau \frac{1-\|x\|^{2}}{1+\langle\tau, x\rangle}\right\rangle .
\end{aligned}
$$

Also, if $z=M_{\tau}(x)$, then

and

$$
1+\langle x, \tau\rangle=1+\left\langle M_{-\tau}(z), \tau\right\rangle=\frac{1-\|\tau\|^{2}}{1-\langle z, \tau\rangle}
$$

$$
1-\|x\|^{2}=1-\left\|M_{-\tau}(z)\right\|^{2}=\sigma(\tau, z) .
$$

Hence by (3.8.24) we obtain

$$
\begin{aligned}
\langle\varphi(x), x\rangle & =\frac{1-\|\tau\|^{2}}{|1-\langle z, \tau\rangle|^{2}}\left\langle g(z), z-\tau \frac{1-\|z\|^{2}}{1-\langle\tau, z\rangle}\right\rangle \\
& =\sigma(\tau, z)\left\langle g(z), \frac{z}{1-\|z\|^{2}}-\frac{\tau}{1-\langle\tau, z\rangle}\right\rangle .
\end{aligned}
$$

Thus (iii) implies

$$
\operatorname{Re}\langle\varphi(z), z\rangle \geq \alpha(\|z\|)\|z\|
$$

Now, if $v(t, z)$ is the solution of the Cauchy problem

$$
\left\{\begin{array}{l}
\frac{\partial v}{\partial t}+\varphi(v)=0 \\
v(0, z)=z
\end{array}\right.
$$

then it follows by Lemma 3.3.2 that $\|v(t, z)\| \leq \beta(t,\|z\|)$. But $v\left(t, M_{-\tau}(x)\right)=M_{-\tau}(u(t, x))$ and this concludes the proof.

REMARK. If $\beta(t, x) \rightarrow 0$ as $t \rightarrow \infty$ for a fixed $s \in[0,1)$, then condition (i) establishes also a rate of convergence of the semigroup $S(t)=u(t, \cdot)$ to its stationary point $\tau \in \mathbb{B}$. It would, of course, be of interest to characterize those functions $\alpha$ for which the convergence of the corresponding semigroup is of exponential type.

3.8.4. Admissible lower and upper bounds and rates of convergence. Here we intend to examine the influence of certain estimates involving the generator on the asymptotic behavior of the semigroup it generates. As in the previous section, we will compare the solutions of the Cauchy problem (3.8.21) with the solutions of certain one-dimensional Cauchy problems, namely,

$$
\left\{\begin{array}{l}
\frac{\partial \beta}{\partial t}+\beta \omega(\beta)=0 \\
\beta(0, s)=s \in[0,1)
\end{array}\right.
$$

We begin with the following two auxiliary assertions. 
LEMMA 3.8.3. Let $\omega$ be a continuous positive function on $[0,1)$. Then for all $s \in[0,1)$, the solution $\beta(t, s)$ of the Cauchy problem (3.8.26) is defined for all $t \geq 0$ and converges to 0 as $t \rightarrow+\infty$. In addition, if $m(s)$ and $M(s)$ are the minimum and maximum, respectively, of the function $\omega$ on the interval $[0, s]$, then

$$
s e^{-M(s) t} \leq \beta(t, s) \leq s e^{-m(s) t} .
$$

Proof. First, rewriting the differential equation of (3.8.26) in the form $\partial \beta / \partial t=-\beta \omega(\beta)$, we note that the solution $\beta(t, s)$ of $(3.8 .26)$ is decreasing with respect to $t$ (when it is defined) and positive. Second, since

$$
\int_{s}^{\beta(t, s)} \frac{d x}{x \omega(x)}=-t
$$

the convergence of the solution $\beta(t, s)$ to a certain limit $s_{0} \geq 0$ as $t \rightarrow \infty$ is equivalent to the divergence of the integral

$$
\int_{s}^{s_{0}} \frac{d x}{x \omega(x)}
$$

But this holds if and only if $s_{0}=0$.

Finally, since

$$
\ln \beta(t, s)-\ln s=-\int_{0}^{t} \omega(\beta(r, s)) d r
$$

the monotonicity of $\beta(\cdot, s)$ implies the last statement of the lemma.

REMARK 1. For a given $0 \leq t<\infty$, consider the two monotone sequences defined as follows:

$$
M_{1}:=M(s)=\max \{\omega(x): x \in[0, s]\}, \quad m_{1}:=m(s)=\min \{\omega(x): x \in[0, s]\}
$$

and

$$
M_{n+1}:=\max \left\{\omega(x): x \in\left[s e^{-M_{n} t}, s\right]\right\}, \quad m_{n+1}:=\min \left\{\omega(x): x \in\left[s e^{-M_{n} t}, s\right]\right\} .
$$

Note that the sequence $\left\{M_{n}\right\}_{n=1}^{\infty}$ is decreasing while the sequence $\left\{m_{n}\right\}_{n=1}^{\infty}$ is increasing. Hence the limits $A=\lim M_{n}$ and $B=\lim m_{n}$ exist. Iterating the proof of the last statement of the preceding lemma we obtain

$$
s e^{-t A} \leq \beta(t, s) \leq s e^{-t B} .
$$

Furthermore, if we have more information about $\omega$, then the latter inequalities can be rewritten in a more precise form, namely, if $\omega$ is increasing, then $A=\omega(s)$ and so $\beta(t, s) \geq s \exp (-t \omega(s))$; if $\omega$ is decreasing, then $B=\omega(s)$ and $\beta(t, s) \leq s \exp (-t \omega(s))$.

LEMMA 3.8.4. Let $\omega_{1}$, and $\omega_{2}$ be two continuous positive functions on $[0,1)$ such that $\omega_{1} \leq \omega_{2}$. Let $\beta_{1}$ and $\beta_{2}$ be the solutions of the following Cauchy problems:

$$
\left\{\begin{array} { l } 
{ \frac { \partial \beta _ { 1 } } { \partial t } + \beta _ { 1 } \omega _ { 1 } ( \beta _ { 1 } ) = 0 , } \\
{ \beta _ { 1 } ( 0 , s ) = s }
\end{array} \quad \text { and } \quad \left\{\begin{array}{l}
\frac{\partial \beta_{2}}{\partial t}+\beta_{2} \omega_{2}\left(\beta_{2}\right)=0 \\
\beta_{2}(0, s)=s
\end{array}\right.\right.
$$


where $s \in[0,1)$. Let $m:[0, \infty) \rightarrow[0,1)$ be a differentiable nonnegative function such that $m(0)=s$ and

$$
-m \omega_{2}(m) \leq \frac{d m}{d t} \leq-m \omega_{1}(m)
$$

Then $\beta_{2}(t, s) \leq m(t) \leq \beta_{1}(t, s)$.

Proof. The assertion is evidently true when $s=0$. Let $t_{0}>0$ be small enough (so that $m(t)>0$ for all $\left.0 \leq t \leq t_{0}\right)$. We have

$$
\int_{s}^{m(t)} \frac{d y}{y \omega_{1}(y)} \leq-t \leq \int_{s}^{m(t)} \frac{d x}{x \omega_{2}(x)}
$$

On the other hand, the definitions of $\beta_{1}$ and $\beta_{2}$ imply that

$$
-t=\int_{s}^{\beta_{1}(t, s)} \frac{d y}{y \omega_{1}(y)}=\int_{s}^{\beta_{2}(t, s)} \frac{d x}{x \omega_{2}(x)}
$$

From this we deduce that

$$
\int_{\beta_{1}(t, s)}^{m(t)} \frac{d y}{y \omega_{1}(y)} \leq 0, \quad \int_{m(t)}^{\beta_{2}(t, s)} \frac{d x}{x \omega_{2}(x)} \leq 0
$$

for $t$ small enough. Since the integrands are positive, we conclude that $\beta_{2}(t, s) \leq m(t) \leq$ $\beta_{1}(t, s)$.

Now we will show that this inequality holds for all $t \geq 0$. Assume that there is $t>0$ such that $m(t)<\beta_{2}(t, s)$. Let $t_{0}$ be the infimum of all such $t$ 's. It is clear that $\beta_{2}\left(t_{0}, s\right)=m\left(t_{0}\right)=s_{0}$. Repeating our arguments, we get $\beta_{2}\left(t+t_{0}, s\right) \leq m\left(t+t_{0}\right)$ for $t$ small enough. But this contradicts the choice of $t_{0}$. In a similar way one can also show that $\beta_{1}(t, s) \geq m(t)$ for all $t \geq 0$.

Proposition 3.8.5. Let $g \in \operatorname{Hol}(\mathbb{B}, H)$ and let $u(=u(t, s))$ be a local solution of the Cauchy problem (3.8.21). Then for any two given continuous functions $\omega_{\ell}$ and $\omega_{u}$ on $[0, q)$ such that $\omega_{\ell}$ is decreasing and nonnegative, and $\omega_{u}$ is increasing, the following assertions are equivalent:

(a) for some $\tau \in \mathbb{B}$ and for each $x \in \mathbb{B}$, there is a number $T=T(x)>0$ such that

$$
\begin{aligned}
\left\|M_{-t}(x)\right\| \exp \left(-t \omega_{u}\left(\left\|M_{-\tau}(x)\right\|\right)\right) & \leq\left\|M_{-\tau}(u(t, x))\right\| \\
& \leq\left\|M_{-\tau}(x)\right\| \exp \left(-t \omega_{\ell}\left(\left\|M_{-\tau}(x)\right\|\right)\right)
\end{aligned}
$$

whenever $t \in[0, T)$;

(b) for some $\tau \in \mathbb{B}$ and for each $x \in \mathbb{B} \backslash\{\tau\}$,

$$
\omega_{\ell}\left(\left\|M_{-\tau}(x)\right\|\right) \leq \frac{\sigma(\tau, x)}{\left\|M_{-\tau}(x)\right\|^{2}} \operatorname{Re}\left\langle g(x), x^{\dagger}\right\rangle \leq \omega_{u}\left(\left\|M_{-\tau}(x)\right\|\right),
$$

where

$$
x^{\dagger}=\frac{1}{1-\|x\|^{2}} x-\frac{1}{1-\langle\tau, x\rangle} \tau \text {. }
$$


When these assertions hold, the point $\tau$ in (a) and (b) is one and the same, and moreover, $g$ is a strongly semicomplete vector field with $g(\tau)=0$. In addition, $u(=u(t, x))$ is globally defined on $\mathbb{R}^{+} \times \mathbb{B}$ and condition (a) holds for all $t \in \mathbb{R}^{+}$.

Proof. Define

$$
\gamma_{u}(t, s)=s e^{-t \omega_{u}(s)}, \quad \gamma_{\ell}(t, s)=s e^{-t \omega_{\ell}(s)}, \quad m_{\tau}(t, x)=\left\|M_{-\tau}(u(t, x))\right\| .
$$

Then (a) can be written as

$$
\gamma_{u}\left(t,\left\|M_{-\tau}(u(t, x))\right\|\right) \leq m_{\tau}(t, x) \leq \gamma_{\ell}\left(t,\left\|M_{-\tau}(u(t, x))\right\|\right), \quad t \in[0, T) .
$$

Since $\gamma_{u}\left(0,\left\|M_{-\tau}(u(t, x))\right\|\right)=m_{\tau}(0, x)=\gamma_{\ell}\left(t,\left\|M_{-\tau}(u(t, x))\right\|\right)$, these inequalities imply that

$$
\frac{\partial \gamma_{u}\left(t,\left\|M_{-\tau}(x)\right\|\right)}{\partial t} \leq \frac{\partial m_{\tau}(t, x)}{\partial t} \leq \frac{\partial \gamma_{\ell}\left(t,\left\|M_{-\tau}(x)\right\|\right)}{\partial t}
$$

at the point $t=0$, or equivalently,

$$
\begin{aligned}
-\omega_{u}\left(\left\|M_{-\tau}(x)\right\|\right) \cdot\left\|M_{-\tau}(x)\right\| & \leq \frac{-\sigma(\tau, x)}{\left\|M_{-\tau}(x)\right\|} \operatorname{Re}\left\langle g(x), x^{\dagger}\right\rangle \\
& \leq-\omega_{\ell}\left(\left\|M_{-\tau}(x)\right\|\right) \cdot\left\|M_{-\tau}(x)\right\| .
\end{aligned}
$$

This yields the implication $(\mathrm{a}) \Rightarrow(\mathrm{b})$. In the other direction, let (b) hold. Then it follows by Proposition 3.8.1 that $g$ is semicomplete with $g(\tau)=0$. Hence the solution of (3.8.21) is globally defined. As above, denote

$$
m(t)=m_{\tau}(t, x)=\left\|M_{-\tau}(u(t, x))\right\|
$$

and $s=\left\|M_{-\tau}(x)\right\|$. Then (b) can be written in the form

$$
-m \omega_{u}(m) \leq \frac{\partial m}{\partial t} \leq-m \omega_{\ell}(m)
$$

which coincides with (3.8.27). As a consequence of Lemma 3.8.4, we have

$$
\beta_{u}(t, s) \leq m(t) \leq \beta_{\ell}(t, s),
$$

where $\beta_{u}$ and $\beta_{\ell}$ are the solutions of the Cauchy problems

$$
\left\{\begin{array} { l } 
{ \frac { \partial \beta _ { u } } { \partial t } + \beta _ { u } \omega _ { u } ( \beta _ { u } ) = 0 , } \\
{ \beta _ { u } ( 0 , s ) = s }
\end{array} \quad \text { and } \quad \left\{\begin{array}{l}
\frac{\partial \beta_{\ell}}{\partial t}+\beta_{\ell} \omega_{\ell}\left(\beta_{\ell}\right)=0 \\
\beta_{\ell}(0, s)=s
\end{array}\right.\right.
$$

This implies the required inequality (a) for all $t \geq 0$ (see Remark 1 after the proof of Lemma 3.8.3).

Definition 3.8.6. Those functions $\omega_{\ell}$ and $\omega_{u}$ which satisfy the assumptions of Proposition 3.8.5 and condition (b) of this proposition will be called admissible lower and upper bounds for $g \in \operatorname{Hol}(\mathbb{B}, H)$ (with respect to the point $\tau \in \mathbb{B}$ ).

Thus the existence of an admissible lower bound for $g$ implies that $g$ is a strongly semicomplete vector field and the flow generated by $g$ is exponentially norm convergent to its stationary point $\tau$, uniformly on each subset strictly inside $\mathbb{B}$.

EXAMPLE 1. The mapping $g(z)=\left(z_{1}, z_{2}\right)$ is obviously a strongly semicomplete vector field on the unit ball in $\mathbb{C}^{2}$ equipped with any norm. Clearly, for small $a \in \mathbb{C}$ the mapping

$$
g_{a}(z)=\left(z_{1}-a z_{2}^{2}, z_{2}\right)
$$


is still a generator. For which $a \in \mathbb{C}$ is it a generator in the domain

$$
D_{p}=\left\{z \in \mathbb{C}^{2}:\left|z_{1}\right|^{p}+\left|z_{2}\right|^{p}<1\right\}, \quad p \geq 1 ?
$$

A related calculation is given in [81]. The answer is the following:

$$
|a| \leq \frac{1}{4^{1 / p}}(p+1)^{1+1 / p}(p-1)^{1 / p-1} .
$$

In the Hilbert ball $\mathbb{B}=D_{2}$ we observe that $g_{a}$ satisfies the estimate

$$
\|z\|^{2}(1-\lambda\|z\|) \leq \operatorname{Re}\left\langle g_{a}(z), z\right\rangle \leq\|z\|^{2}(1+\lambda\|z\|),
$$

where $\lambda=|a| \frac{2}{3 \sqrt{3}} \in[0,1]$. Those inequalities coincide exactly with condition (b) of Proposition 3.8.5 for the admissible lower and upper bounds defined by

$$
\omega_{\ell}(s)=1-\lambda s \quad \text { and } \quad \omega_{u}(s)=1+\lambda s .
$$

Hence by this proposition we obtain the following estimate for the semigroup $\{u(t, \cdot)\}$ generated by $g_{a}$ :

$$
\|z\| e^{-t(1+\lambda\|z\|)} \leq\|u(t, z)\| \leq\|z\| e^{-t(1-\lambda\|z\|)} .
$$

REMARK 1. If $\varrho$ is the Poincaré metric on $\mathbb{B}$, then using the known (see [30]) properties of $\varrho$ :

$$
\varrho(x, y)=\varrho\left(0, M_{-y}(x)\right), \quad \varrho(0, k x) \leq k \varrho(0, x), \quad 0 \leq k \leq 1,
$$

we see that condition (a) in Proposition 3.8.5 implies the inequality

$$
\varrho(u(t, x), \tau) \leq e^{-t \omega\left(\left\|M_{-\tau}(x)\right\|\right)} \varrho(x, \tau) .
$$

In other words, the exponential norm convergence implies the same rate of convergence in the hyperbolic metric.

REMARK 2. Generally speaking, the converse is not clear, because we know only local estimates: For each $x \in \mathbb{B}$ there are a neighborhood $U$ of $x$ and numbers $M=M(x, U) \geq 1$ and $m=m(x, U) \leq 1$ such that

$$
m\|x-y\| \leq \varrho(x, y) \leq M\|x-y\|
$$

for all $y \in U$ (see [30]). Therefore the following question arises: are there an admissible lower bound $\omega_{\ell}$ and an admissible upper bound $\omega_{u}$ for $g \in N_{\tau}$ such that condition (b) of Proposition 3.8.5 is equivalent to the same or a similar estimate of convergence in the metric $\varrho$ ? We answer this question by establishing the following assertion.

Proposition 3.8.7. Let $g \in \operatorname{Hol}(\mathbb{B}, H)$ and let $u=u(t, x)$ be the local solution of the Cauchy problem (3.8.21). Let $\omega_{u}$ and $\omega_{\ell}$ be continuous positive functions defined on $[0,1)$ such that

$$
\begin{cases}\omega_{u}(s) & \text { is increasing } \\ \frac{\omega_{\ell}(s) s}{\left(1-s^{2}\right) \operatorname{arctanh} s} & \text { is decreasing. }\end{cases}
$$

Then the following assertions are equivalent:

(a) $\omega_{u}$ is an admissible upper bound and $\omega_{\ell}$ is an admissible lower bound (with respect to some point $\tau \in \mathbb{B})$ for $g$; 
(b) for some $\tau \in \mathbb{B}$ and for each $x \in \mathbb{B}$, there is $T=T(x)$ such that

$$
\begin{aligned}
& \varrho(\tau, x) \exp \left(-t \omega_{u}\left(\left\|M_{-\tau}(x)\right\|\right) \frac{\left\|M_{-\tau}(x)\right\|}{\varrho(\tau, x) \sigma(\tau, x)}\right) \leq \varrho(\tau, u(t, x)) \\
& \quad \leq \varrho(\tau, x) \exp \left(-t \omega_{\ell}\left(\left\|M_{-\tau}(x)\right\|\right) \frac{\left\|M_{-\tau}(x)\right\|}{\varrho(\tau, x) \sigma(\tau, x)}\right),
\end{aligned}
$$

whenever $t \in[0, T)$. The point $\tau$ in (a) and (b) is one and the same. Moreover, for each $x \in \mathbb{B}$, the solution $u(\cdot, x)$ is well defined globally and (b) holds for all $t \geq 0$.

Proof. It is clear that condition (a) coincides with condition (b) of Proposition 3.8.5. As above, consider the function

$$
m(t)=\left\|M_{-\tau}(u(t, x))\right\| .
$$

If (a) holds, then it implies that

$$
-m \omega_{u}(m) \leq \frac{d m}{d t} \leq-m \omega_{\ell}(m) .
$$

By Lemma 3.8.4, we obtain

$$
\beta_{u}(t) \leq m(t) \leq \beta_{\ell}(t)
$$

where $\beta_{u}$ and $\beta_{\ell}$ are the solutions of the following Cauchy problems:

$$
\left\{\begin{array} { l } 
{ \frac { \partial \beta _ { u } } { \partial t } + \beta _ { u } \omega _ { u } ( \beta _ { u } ) = 0 , } \\
{ \beta _ { u } ( 0 ) = s = \| M _ { - \tau } ( x ) \| }
\end{array} \quad \text { and } \quad \left\{\begin{array}{l}
\frac{\partial \beta_{\ell}}{\partial t}+\beta_{\ell} \omega_{\ell}\left(\beta_{\ell}\right)=0 \\
\beta_{e}(0)=s=\left\|M_{-\tau}(x)\right\| .
\end{array}\right.\right.
$$

This implies that

$$
\ln \left(\frac{\operatorname{arctanh} \beta_{u}}{\operatorname{arctanh} s}\right)=-\int_{0}^{t} \frac{\beta_{u}(r) \omega_{u}\left(\beta_{u}(r)\right) d r}{\left(1-\beta_{u}^{2}(r)\right) \operatorname{arctanh} \beta_{u}(r)}
$$

and the same equality holds for $\beta_{\ell}$ and $\omega_{\ell}$. Now by using (3.8.29) and (3.8.30), we get condition (b). The implication $(b) \Rightarrow(a)$ follows from the comparison of the derivatives in inequality (b) with respect to $t$ at the point $t=0^{+}$.

Now we will show that there are "universal" admissible lower and upper bounds $\omega_{\wedge}$ and $\omega^{\wedge}$.

Proposition 3.8.8. For $g \in \operatorname{Hol}(\mathbb{B}, H)$ and any point $\tau \in \mathbb{B}$, the following assertions are equivalent:

(i) $g$ has an admissible lower bound $\omega_{\ell}$ and an admissible upper bound $\omega_{u}$ with respect to the point $\tau \in \mathbb{B}$;

(ii) $g \in N_{\tau}$ and there are numbers $a \geq 0$ and $b \geq a$ such that

$$
\begin{aligned}
\left\|M_{-\tau}(x)\right\| \exp \left(-t b \frac{1+\left\|M_{-\tau}(x)\right\|}{1-\left\|M_{-\tau}(x)\right\|}\right) & \leq\left\|M_{-\tau}(u(t, x))\right\| \\
& \leq\left\|M_{-\tau}(x)\right\| \exp \left(-t a \frac{1-\left\|M_{-\tau}(x)\right\|}{1+\left\|M_{-\tau}(x)\right\|}\right),
\end{aligned}
$$

where $u(t, x)$ is the solution of the Cauchy problem (3.8.21); 
(iii) $g \in N_{\tau}$ and there are numbers $a \geq 0$ and $b \geq a$ such that

$$
\begin{aligned}
& \varrho(\tau, x) \exp \left(-\tau b \frac{\left\|M_{-\tau}(x)\right\|}{\left(1-\left\|M_{-\tau}(x)\right\|\right)^{2} \varrho(\tau, x)}\right) \leq \varrho(\tau, u(t, x)) \\
& \leq \varrho(\tau, x) \exp \left(-t a \frac{\left\|M_{-\tau}(x)\right\|}{\left(1+\left\|M_{-\tau}(x)\right\|\right)^{2} \varrho(\tau, x)}\right) .
\end{aligned}
$$

The numbers $a$ and $b$ in (ii) and (iii) can be chosen to be the same. In particular, if (i) holds, then one can put $a=\omega_{\ell}(0)$ and $b=\omega_{u}(0)$.

Proof. We intend to show that (i) is equivalent to

(i') The functions

$$
\omega_{\wedge}(s)=a \frac{1-s}{1+s} \quad \text { and } \quad \omega^{\wedge}(s)=b \frac{1+s}{1-s}
$$

(with some numbers $a$ and $b$ ) are admissible lower and upper bounds.

In fact, all we need to prove is that $(\mathrm{i}) \Rightarrow\left(\mathrm{i}^{\prime}\right)$, since the reverse implication is evident.

Set $v(t, x)=M_{-\tau}\left(u\left(t, M_{\tau}(x)\right)\right)$ and $\varphi(x)=-\partial v\left(0^{+}, x\right) / \partial t$. If $g \in \operatorname{Hol}(\mathbb{B}, H)$ has admissible lower and upper bounds $\omega_{\ell}$ and $\omega_{u}$, then by Proposition 3.8.5, v(t,x) is well defined for all $t \geq 0$ and

$$
\|x\| \exp \left(-t \omega_{u}(\|x\|)\right) \leq\|v(t, x)\| \leq\|x\| \exp \left(-t \omega_{\ell}(\|x\|)\right) .
$$

Again by Proposition 3.8.5 we see that

$$
\omega_{\ell}(\|x\|) \leq \frac{1}{\|x\|^{2}} \operatorname{Re}\langle\varphi(x), x\rangle \leq \omega_{u}(\|x\|) .
$$

This implies that $\varphi(0)=0$ and that

$$
\omega_{\ell}(0) \leq \frac{1}{\|x\|^{2}} \operatorname{Re}\left\langle\varphi^{\prime}(0) x, x\right\rangle \leq \omega_{u}(0) .
$$

Now it follows from Proposition 3.5.2 that we also have

$$
a \frac{1-\|x\|}{1+\|x\|} \leq \frac{1}{\|x\|^{2}} \operatorname{Re}\langle\varphi(x), x\rangle \leq b \frac{1+\|x\|}{1-\|x\|}
$$

when $0 \leq a \leq \omega_{\ell}(0)$ and $b \geq \omega_{u}(0)$. This means that the functions

$$
\omega_{\wedge}(s)=a \frac{1-s}{1+s} \quad \text { and } \quad \omega^{\wedge}(s)=b \frac{1+s}{1-s}
$$

are also admissible lower and upper bounds for $\varphi$ (and consequently for $g$ ). It is easy to verify that the function $\omega^{\wedge}$ is increasing and that the function $\omega_{\wedge}(s) s /\left(1-s^{2}\right) \operatorname{arctanh} s$ is decreasing.

Finally, (i') is equivalent to condition (ii) by Proposition 3.8.5 and to (iii) by Proposition 3.8.7.

REMARK. Actually, conditions (i)-(iii) of this proposition are equivalent to the following one: $g \in N_{\tau}$ and there are numbers $0 \leq a \leq b$ such that

$$
a\|B x\|^{2} \leq \operatorname{Re}\left\langle B g^{\prime}(\tau) x, B x\right\rangle \leq b\|B x\|^{2}
$$

for all $x \in H$, where $B$ is the linear operator defined by $B=P_{\tau}+\sqrt{1-\|\tau\|^{2}}\left(I-P_{\tau}\right)$, $\tau \in \mathbb{B} \backslash\{0\}, P_{\tau}=\tau\langle\cdot, \tau\rangle /\|\tau\|^{2}$ and $B=I$ when $\tau=0$. Indeed, by direct calculations 
one can show that $\varphi^{\prime}(0)=B g^{\prime}(\tau) B^{-1}$ and (3.8.31) becomes (3.8.33). In addition, using Proposition 3.8.5 and substituting the admissible lower and upper bounds (3.8.32), we conclude that the above conditions of Proposition 3.8.8 including (3.8.33) are equivalent to the inequality

$$
a\left(\frac{\left\|M_{-\tau}(x)\right\|}{1+\left\|M_{-\tau}(x)\right\|}\right)^{2} \leq \operatorname{Re}\left\langle g(x), x^{\dagger}\right\rangle \leq b\left(\frac{\left\|M_{-\tau}(x)\right\|}{1-\left\|M_{-\tau}(x)\right\|}\right)^{2}
$$

(compare with $\left.\left(i^{\prime}\right)\right)$.

3.8.5. A parametric representation of semicomplete vector fields with an interior null point. Now we are able to present a generalization of the Berkson-Porta representation for the class $N_{\tau}, \tau \in \mathbb{B}$.

Proposition 3.8.9. A mapping $g \in \operatorname{Hol}(\mathbb{B}, H)$ belongs to $N_{\tau}$ if and only if there is a mapping $Q \in \operatorname{Hol}(\mathbb{B}, L(H)$ ) (i.e., for each $x \in H, Q(x)$ is a bounded linear operator $Q(x): H \rightarrow H$ which depends holomorphically on $x \in \mathbb{B})$ such that

$$
\begin{gathered}
g(x)=(I-x\langle\cdot, \tau\rangle) Q(x)(x-\tau), \\
\operatorname{Re}\langle Q(x)(x-\tau),(x-\tau)\rangle \geq 0 .
\end{gathered}
$$

Proof. We denote by $\Lambda(x)$ the mapping $\Lambda(x):=(I-x\langle\cdot, \tau\rangle) \in L(H)$. It is clear that for each $x \in \mathbb{B}$ and $\tau \in \mathbb{B}$, the linear operator $\Lambda(x)$ is continuously invertible. Therefore, for each $g \in \operatorname{Hol}(\mathbb{B}, H)$, the mapping $f: \mathbb{B} \rightarrow H$, defined by

$$
f(x)=[\Lambda(x)]^{-1} g(x),
$$

belongs to $\operatorname{Hol}(\mathbb{B}, H)$, and if $g(\tau)=0$ for some $\tau \in \mathbb{B}$, then $f(\tau)=0$ too.

Now by the integral form of the mean value theorem we get

$$
\begin{aligned}
f(x)=f(x)-f(\tau) & =\int_{0}^{1} \frac{d}{d t}[f(\tau+t(x-\tau))] d t \\
& =\left[\int_{0}^{1} f^{\prime}(\tau+t(x-\tau)) d t\right](x-\tau)=Q(x)(x-\tau),
\end{aligned}
$$

where for each $x \in \mathbb{B}, Q(x)=\int_{0}^{1} f^{\prime}(\tau+t(x-\tau)) d t$ is a linear operator from $H$ to $H$ which depends holomorphically on $x \in \mathbb{B}$. Moreover, since the segment $[x, \tau]$ is a compact subset of $\mathbb{B}$, the operator $Q(x)$ is bounded for each $x \in \mathbb{B}$, that is, $Q(x) \in L(H)$. In other words, we have shown that each $g \in \operatorname{Hol}(\mathbb{B}, H)$ vanishing at $\tau \in \mathbb{B}$ can be represented by

$$
g(x)=\Lambda(x) f(x)=(I-x\langle\cdot, \tau\rangle) Q(x)(x-\tau),
$$

where $Q(x) \in \operatorname{Hol}(\mathbb{B}, L(H))$.

Now we have by (3.8.34),

$$
\begin{aligned}
\operatorname{Re}\left\langle g(x), x^{\dagger}\right\rangle & =\operatorname{Re}\left\langle\Lambda(x) f(x), x^{\dagger}\right\rangle=\left\langle\operatorname{Re} f(x),[\Lambda(x)]^{*} x^{\dagger}\right\rangle \\
& =\operatorname{Re}\left\langle Q(x)(x-\tau),[\Lambda(x)]^{*} x^{\dagger}\right\rangle,
\end{aligned}
$$

where $[\Lambda(x)]^{*}$ denotes the adjoint of $\Lambda(x)$,

$$
[\Lambda(x)]^{*}=(I-\tau\langle\cdot, x\rangle),
$$


and $x^{\dagger}$ denotes (as above) the support functional of the ellipsoid $E_{\tau}$ at the point $x$, that is,

$$
x^{\dagger}=\frac{x}{1-\|x\|^{2}}-\frac{\tau}{1-\langle\tau, x\rangle} .
$$

But $[\Lambda(x)]^{*} x^{\dagger}=(x-\tau) /\left(1-\|x\|^{2}\right)$ and (3.8.35) implies that

$$
\operatorname{Re}\left\langle g(x), x^{\dagger}\right\rangle=\frac{1}{1-\|x\|^{2}} \operatorname{Re}\langle Q(x)(x-\tau), x-\tau\rangle .
$$

Now our assertion follows from Proposition 3.8.1.

REMARK 1. It is clear that for the one-dimensional case, conditions (a) and (b) of Proposition 3.8.9 become the Berkson-Porta representation of a semicomplete vector field with $\tau \in \Delta$.

REMARK 2. Using (3.8.34) and (3.8.38), one can rephrase some assertions in Sections 3.8.1-3.8.4. For example, let $g \in \operatorname{Hol}(\mathbb{B}, H)$ and let $u(t, x)$ be the local solution of the Cauchy problem (3.8.21). Then by Proposition 3.8.8 the following assertions are equivalent:

(i) for some $\tau \in \mathbb{B}$ and $Q \in \operatorname{Hol}(\mathbb{B}, L(H))$,

$$
g(x)=(I-x\langle\cdot, \tau\rangle) Q(x)(x-\tau)
$$

with

$$
\operatorname{Re}\langle Q(x)(x-\tau),(x-\tau)\rangle \geq a\left(1-\|x\|^{2}\right) \frac{\left\|M_{-\tau}(x)\right\|^{2}}{1-\left\|M_{-\tau}(x)\right\|^{2}}
$$

for some $a \geq 0$

(ii) $g \in N_{\tau},\{u(t, x)\} \subset \mathbb{B}$ is well-defined for all $(t, x) \in \mathbb{R}^{+} \times \mathbb{B}$, and for some $a \geq 0$,

$$
\left\|M_{-\tau}(u(t, x))\right\| \leq e^{-a t \frac{1-\left\|M_{-\tau}(x)\right\|}{1+\left\|M_{-\tau}(x)\right\|}}\left\|M_{-\tau}(x)\right\| .
$$

The number a in (i) and (ii) can be chosen the same.

In particular, suppose that the operator $Q(x) \in L(H)$ in (3.8.34) is a strongly accretive operator, uniformly for $x \in \mathbb{B}$, i.e., there is $c>0$ such that for each $x \in \mathbb{B}$ and $y \in H$,

$$
\operatorname{Re}\langle Q(x) y, y\rangle \geq c\|y\|^{2} .
$$

Then we claim that condition (ii) holds with $a=c\left(1-\|\tau\|^{2}\right)$.

Indeed, (3.8.39) implies that

$$
\begin{aligned}
\operatorname{Re}\langle Q(x)(x-\tau),(x-\tau)\rangle & \geq c\|x-\tau\|^{2} \geq c|1-\langle x, \tau\rangle|^{2} \cdot\left\|M_{-\tau}(x)\right\|^{2} \\
& =a\left\|M_{-\tau}(x)\right\|^{2} \cdot \frac{|1-\langle x, \tau\rangle|^{2}}{1-\|\tau\|^{2}} \cdot \frac{\left(1-\|x\|^{2}\right)}{1-\|x\|^{2}} \\
& =a\left(1-\|x\|^{2}\right) \frac{\left\|M_{-\tau}(x)\right\|^{2}}{\sigma(x, \tau)}=a\left(1-\|x\|^{2}\right) \frac{\left\|M_{-\tau}(x)\right\|^{2}}{1-\left\|M_{-\tau}(x)\right\|^{2}} .
\end{aligned}
$$

So we have condition (i) above, hence (ii), and we are done. 
As a matter of fact, we have more precise information in this case. Namely, the last inequality is actually equivalent to

$$
\operatorname{Re}\left\langle g(x), x^{\dagger}\right\rangle \geq \frac{a\left\|M_{-\tau}(x)\right\|^{2}}{\sigma(x, \tau)} .
$$

Therefore if we set $\omega_{\ell}(s)=a$ in Proposition 3.8.5 we get

$$
\left\|M_{-\tau} u(t, x)\right\| \leq e^{-a t}\left\|M_{-\tau}(x)\right\|,
$$

i.e., we have a uniform exponential rate of convergence for all $x \in \mathbb{B}$.

3.8.6. A parametric representation of semicomplete vector fields with no null point

Proposition 3.8.10. Let now $g \in \mathcal{G}(\mathbb{B})$ have no null point in $\mathbb{B}$. Then there is a point $\tau \in \partial \mathbb{B}$ such that $g$ admits the representation

$$
g(x)=(I-x\langle\cdot, \tau\rangle) f(x)
$$

where $f \in \operatorname{Hol}(\mathbb{B}, H)$ with

$$
\operatorname{Re}\langle f(x), x-\tau\rangle \geq 0
$$

for all $x \in \mathbb{B}$.

Proof. Consider the mappings $g_{n}$ :

$$
g_{n}(x):=g(x)+\frac{1}{n} x, \quad n=1,2, \ldots
$$

Since $\mathcal{G}$ is a real cone, $g_{n} \in \mathcal{G}(\mathbb{B})$. Moreover, we claim that for each $n$, there is $\tau_{n} \in \mathbb{B}$ such that $g_{n} \in N_{\tau_{n}}$ and the sequence $\left\{\tau_{n}\right\}$ strongly converges to a point $\tau \in \partial \mathbb{B}$. Indeed, the equation $g_{n}(x)=0$ is equivalent to the equation

$$
(I+n g)(x)=0 .
$$

If we denote by $J_{r}$ the resolvent $(I+r g)^{-1}: \mathbb{B} \rightarrow \mathbb{B}$ of $g$, then we see that (3.8.43) has a unique solution: $\tau_{n}=J_{n}(0)$. Since $g$ has no null point in $\mathbb{B}$, it follows that for each $x \in \mathbb{B}$, the net $\left\{J_{r}(x)\right\}$ converges to a point $\tau \in \partial \mathbb{B}[68$, p. 248]. In particular, the sequence $\left\{\tau_{n}\right\}$ strongly converges to $\tau$. The claim is proved.

Now, using Proposition 3.8.9, we check that each $g_{n}$ admits the representation

$$
g_{n}(x)=\Lambda_{n} f_{n}(x),
$$

where $\Lambda_{n}=I-x\left\langle\cdot, \tau_{n}\right\rangle$ and $f_{n}(x):=Q_{n}(x)\left(x-\tau_{n}\right)$. Hence

$$
\operatorname{Re}\left\langle f_{n}(x), x-\tau_{n}\right\rangle \geq 0 .
$$

Observe also that since for each $x \in \mathbb{B}$,

$$
\left\|I-x\left\langle\cdot, \tau_{n}\right\rangle\right\| \geq 1-\|x\|,
$$

the operators $\Lambda_{n}^{-1}\left(=\left(I-x\left\langle\cdot, \tau_{n}\right\rangle\right)^{-1}\right)$ are uniformly bounded and consequently converge to $\Lambda^{-1}=(I-x\langle\cdot, \tau\rangle)^{-1}$. Also, (3.8.42) implies that for each $x \in \mathbb{B}$ the sequence $\left\{g_{n}(x)\right\}$ converges to $g(x)$. Hence the sequence $\left\{f_{n}(x)\right\}=\left\{\Lambda_{n}^{-1} g_{n}(x)\right\}$ converges to $f(x)=\Lambda^{-1} g(x)$. It is clear that $f \in \operatorname{Hol}(\mathbb{B}, H)$. This fact and inequality (3.8.45) immediately imply our assertion. 
Proposition 3.8.11. Let $g \in \operatorname{Hol}(\mathbb{B}, H)$ admit the representation (3.8.40) with (3.8.41) and $\tau \in \partial \mathbb{B}$. Assume also that

$$
\operatorname{Re}\langle f(x), \tau\rangle \geq m
$$

for some real $m$. Then $g \in \mathcal{G}(\mathbb{B})$ and $\tau$ is a sink point for $g$.

Proof. By simple calculations we get for $x \in \mathbb{B}$,

$$
\begin{aligned}
\operatorname{Re}\langle g(x), x\rangle & =\operatorname{Re}\langle(I-x\langle\cdot, \tau\rangle) f(x), x\rangle=\operatorname{Re}\left\langle f(x),(I-x\langle\cdot, \tau\rangle)^{*} x\right\rangle \\
& =\operatorname{Re}\langle f(x),(I-\tau\langle\cdot, x\rangle) x\rangle=\operatorname{Re}\left\langle f(x), x-\tau\|x\|^{2}\right\rangle \\
& =\operatorname{Re}\left[\langle f(x), x-\tau\rangle+\left\langle f(x), \tau\left(1-\|x\|^{2}\right)\right\rangle\right] \geq m\left(1-\|x\|^{2}\right) .
\end{aligned}
$$

Applying Proposition 3.5.2, we conclude that $g \in \mathcal{G}(\mathbb{B})$.

Now to prove that $\tau$ is a sink point for $g$ we have to show that if $S(t) x$ is the solution of the Cauchy problem (3.8.21), then each ellipsoid $E(\tau, k)=\left\{x \in \mathbb{B}: \varphi_{\tau}(x)<K\right\}$, where

$$
\varphi_{\tau}(x)=\frac{|1-\langle x, \tau\rangle|^{2}}{1-\|x\|^{2}},
$$

which is internally tangent to the boundary of $\mathbb{B}$ at $\tau$ is $S(t)$-invariant. To this end, consider the function

$$
\Psi(t, x)=\varphi_{\tau}(S(t) x) .
$$

By direct calculations it can be shown that

$$
\frac{\partial \Psi\left(0^{+}, x\right)}{\partial t}=-2 \Psi(0, x) \cdot \operatorname{Re}\left\langle g(x), x^{\dagger}\right\rangle,
$$

where $x^{\dagger}=x /\left(1-\|x\|^{2}\right)-\tau /(1-\langle\tau, x\rangle)$. Also, it follows by the semigroup property that

$$
\Psi(s+t, x)=\Psi(t, S(s) x), \quad \Psi(s x)=\Psi(0, S(s) x) .
$$

Hence,

$$
\begin{aligned}
\frac{\partial \Psi(s, x)}{\partial s} & =-2 \Psi(s, x) \operatorname{Re}\left\langle g(S(s) x),[S(s) x]^{\dagger}\right\rangle \\
& =-2 \Psi(s, x) \operatorname{Re}\langle f(S(s) x), S(s) x-\tau\rangle \frac{1}{1-\|S(s) x\|^{2}}
\end{aligned}
$$

Now (3.8.41) and (3.8.47) imply that $\Psi(\cdot, x)$ is a decreasing function on $[0, \infty)$. In other words,

$$
\varphi_{\tau}(S(t) x) \leq \varphi_{\tau}(x)
$$

for each $x \in \mathbb{B}$ and all $t \geq 0$. The proof is complete.

In our next proposition we use formulas (3.8.47) and (3.8.48) to find a sufficient condition for the convergence of the semigroup generated by $g$ to a boundary point when $g$ has no null point in $\mathbb{B}$.

Proposition 3.8.12. Let $g \in \mathcal{G}(\mathbb{B})$ satisfy the condition

$$
\operatorname{Re}\left\langle g(x), \frac{x}{1-\|x\|^{2}}-\frac{\tau}{1-\langle\tau, x\rangle}\right\rangle \geq \omega\left(\varphi_{\tau}(x)\right)
$$


for some $\tau \in \partial \mathbb{B}$ and for all $x \in \mathbb{B}$, where $\omega$ is a decreasing positive function on $(0, \infty)$ and

$$
\varphi_{\tau}(x)=\frac{|1-\langle x, \tau\rangle|^{2}}{1-\|x\|^{2}} .
$$

Then $g$ has no null point in $\mathbb{B}$ and for each $x \in \mathbb{B}$,

$$
\varphi_{\tau}(S(t) x) \leq e^{-2 \omega\left(\varphi_{\tau}(x)\right) t} \varphi_{\tau}(x),
$$

where $\{S(t)\}$ is the semigroup generated by $g$. In other words,

$$
|1-\langle S(t) x, \tau\rangle|^{2} \leq \exp \left(-2 t \omega\left(\frac{|1-\langle x, \tau\rangle|^{2}}{1-\|x\|^{2}}\right)\right) \frac{|1-\langle x, \tau\rangle|^{2}\left(1-\|S(t) x\|^{2}\right)}{1-\|x\|^{2}} .
$$

Proof. If we again set $\Psi(t, x)=\varphi_{\tau}(S(t) x)$, then it follows by (3.8.47) and (3.8.49) that

$$
\frac{1}{\Psi(t, x)} \frac{\partial \Psi(t, x)}{\partial t}=-2 \operatorname{Re}\left\langle g\left(S(t) x,[S(t) x]^{\dagger}\right\rangle \geq-2 \omega(\Psi(t, x)) .\right.
$$

Integrating this inequality and using (3.8.48), we get

$$
\Psi(t, x) \leq \Psi(0, x) \exp \left(-2 \int_{0}^{t} \omega(\Psi(r, x)) d r\right) \leq \Psi(0, x) \exp (-2 \omega(\Psi(0, x)) t)
$$

because $\omega$ is decreasing. Thus (3.8.50) and hence (3.8.51) follow. This also implies that $g$ has no null point in $\mathbb{B}$, because otherwise such a point would be a stationary point of $\{S(t)\}$ and (3.8.50) would be impossible.

In particular, if there exists $a>0$ such that for each $x \in \mathbb{B}$, the condition $\operatorname{Re}\left\langle g(x), x^{\dagger}\right\rangle$ $\geq a$ is fulfilled, then (3.8.51) implies

$$
\|S(t) x-\tau\|^{2} \leq \frac{2 e^{-a t}\|x-\tau\|}{\sqrt{1-\|x\|^{2}}} .
$$

Moreover, since $\|S(t) x\| \rightarrow 1$ as $t \rightarrow \infty$, for sufficiently large $t$ we can deduce from (3.8.51) a sharper estimate:

$$
\|S(t) x-\tau\|^{2}<e^{-a t}\|x-\tau\|, \quad t>T .
$$

EXAmple 1. Let $\Delta$ be the open unit disk in the complex plane $\mathbb{C}$ and let

$$
g(z)=-(1-z)^{2} p(z)
$$

where $p \in \operatorname{Hol}(\Delta, \mathbb{C})$ with $\operatorname{Re} p(z) \geq a\left(1-|z|^{2}\right) /|1-z|^{2}$ for some $a>0$ and all $z \in \Delta$. (For instance, we can take $p(z)=\left(1-|z|^{2}\right) /|1-z|^{2}, z \in \Delta$.) Then setting $\tau=1$ we get

$$
\begin{aligned}
\operatorname{Re} g(z)\left(\frac{\bar{z}}{1-|z|^{2}}-\frac{1}{1-z}\right) & =\operatorname{Re} \frac{g(z)\left(\bar{z}-|z|^{2}-1+|z|^{2}\right)}{\left(1-|z|^{2}\right)(1-z)} \\
& =\frac{-1}{1-|z|^{2}} \operatorname{Re}(1-z)(\bar{z}-1) p(z)=\frac{|1-z|^{2}}{1-|z|^{2}} \operatorname{Re} p(z) \geq a
\end{aligned}
$$

Thus we have estimates (3.8.51) with $\omega \equiv a$, as well as (3.8.52) and (3.8.53).

ExAmPle 2. Let $g \in \operatorname{Hol}(\Delta, \mathbb{C})$ be given by (3.8.54) with

$$
\operatorname{Re} p(z) \geq a>0 \text {. }
$$


For instance, $p(z)=(1-c z) /(1+c z), c \in(0,1)$. If we define $\omega(s)=a / s, s>0$, then we get (as above)

$$
\operatorname{Re} g(z)\left(\frac{\bar{z}}{1-\|z\|^{2}}-\frac{1}{1-z}\right) \geq \frac{a}{\varphi_{1}(z)}=\omega\left(\varphi_{1}(z)\right) .
$$

Now by (3.8.51) we deduce the following fact: if

$$
z \in E(k, 1)=\left\{z \in \Delta: \varphi_{1}(z)<k\right\}
$$

then

$$
|S(t) z-1| \leq e^{-\frac{a}{k} t} \sqrt{k}
$$

\subsection{Growth and covering theorems for star-like mappings on the unit ball of}

Banach and Hilbert spaces. A celebrated theorem of Köbe asserts that the image of any univalent function on the unit disk of the form $z+\sum_{k=2}^{\infty} a_{k} z^{k}$ covers the disk of radius $1 / 4$ centered at the origin. This fact is no longer true in higher dimensions (see [17]). Nevertheless, Barnard, FitzGerald and Gong showed that a similar assertion can be established for star-like mappings on the unit ball of a Banach space, normalized at the origin (see [12]). An improved result was obtained in [19] for the so-called strongly star-like mappings. Actually, covering estimates (as well as growth estimates) turn out to depend on lower and upper admissible bounds for semicomplete vector fields related to star-like mappings.

Let $D$ be (as above) a domain in a Banach space $X$ and let $f \in \operatorname{Hol}(D, X)$. We recall that $f$ is star-like on $D$ (with respect to an interior point $\tau$ ), i.e., $f \in S_{\tau}^{*}(D)$, if and only if it satisfies the differential equation

$$
f(x)=f^{\prime}(x) g(x)
$$

for some strongly semicomplete vector field $g$ on $D$ such that

$$
\begin{gathered}
g(\tau)=0, \\
g^{\prime}(\tau)=I .
\end{gathered}
$$

We will call such a mapping $g$ the characteristic mapping of $f$, and the evolution equation

$$
\left\{\begin{array}{l}
\frac{\partial u(t, x)}{\partial t}+g(u(t, x))=0 \\
u(0, x)=x \in D
\end{array}\right.
$$

will be called the characteristic equation for $f$ defined by (3.9.1). Recall also that if $D$ is bounded, then $f$ can be found by the formula (see Proposition 3.7.5)

$$
f(x)=A \lim _{t \rightarrow \infty} e^{t}[u(t, x)-\tau]
$$

where $A=f^{\prime}(\tau)$ and there is a ball $B \subseteq D$ centered at $\tau$ such that $f$ is star-like on $B$. Set $f_{1}(x)=A^{-1} f(x), x \in D$. Then $f_{1}$ also satisfies (3.9.1) and is star-like on both $D$ and $B$. So, one can try to establish growth and covering estimates for both $f$ and $f_{1}$ on $B$. If, in addition, $D$ is a symmetric domain, then it can be realized as the unit ball in a $J B^{*}$-triple system, and we are able to study the behavior of these mappings on the whole of $D$ by using similar tools to the ones we employed in a Hilbert space. Indeed, first we note that in this case the domain $D$ is homogeneous, i.e., for each $x \in D$ there is 
an automorphism $M_{-\tau} \in \operatorname{Aut}(D)$ such that $M_{-\tau}(\tau)=0$. The mapping $\tilde{f}=f_{1} \circ M_{-\tau}$ is also star-like, since $\widetilde{f}(D)=f_{1}(D)=A^{-1} f(D)$.

At the same time, Lemma 3.7.1 implies that the mapping

$$
\widetilde{g}=\left(\left[M_{-\tau}(\cdot)\right]^{\prime}\right)^{-1}\left[g\left(M_{\tau}(\cdot)\right)\right]
$$

belongs to $\mathcal{G}(D)$ and is normalized by

$$
\begin{gathered}
\widetilde{g}(0)=0, \\
\widetilde{g}^{\prime}(0)=I .
\end{gathered}
$$

Hence, by the chain rule and (3.9.1), we get

$$
\begin{aligned}
\tilde{f}(x) & =f\left(M_{-\tau}(x)\right)=f^{\prime}\left(M_{-\tau}(x)\right)\left[g\left(M_{-\tau}(x)\right)\right] \\
& =\widetilde{f}^{\prime}(x)\left[\left[M_{-\tau}(x)\right]^{\prime}\right]^{-1}\left[g\left(M_{-\tau}(x)\right)\right]=\widetilde{f}^{\prime}(x) \widetilde{g}(x) .
\end{aligned}
$$

Thus $\widetilde{g}$ is the characteristic mapping for $\widetilde{f}$ and

$$
v(t, x)=M_{-\tau}\left(u\left(t, M_{\tau}(x)\right)\right)
$$

is the solution of the characteristic equation for $\tilde{f}$.

Let now $\omega=\left(\omega_{1}, \omega_{2}\right)$ be a pair of positive real functions on $[0,1)$ such that the Cauchy problems

$$
\left\{\begin{array}{l}
\frac{\partial \beta_{i}(t, s)}{\partial t}+\beta_{i}(t, s) \omega_{i}\left(\beta_{i}(t, s)\right)=0 \\
\beta_{i}(0, s)=s \in[0,1), \quad i=1,2
\end{array}\right.
$$

have unique solutions $\beta_{i}(t, s) \in[0,1)$ for all $t \geq 0, s \in[0,1)$. Assume also that $\omega_{1}$ is an increasing function while $\omega_{2}$ is decreasing.

We will say that $f$ is $\omega$-star-like on $D$ if $\omega_{1}$ and $\omega_{2}$ are admissible lower and upper bounds for $\widetilde{g}$, i.e., for each $x \in D$ and $x^{*} \in J(x)$,

$$
\omega_{2}(\|x\|)\|x\|^{2} \geq \operatorname{Re}\left\langle\widetilde{g}(x), x^{*}\right\rangle \geq \omega_{1}(\|x\|)\|x\|^{2}
$$

(cf. Definition 3.8.6). Since $\widetilde{g}^{\prime}(0)=I$, the existence of $\omega=\left(\omega_{1}, \omega_{2}\right)$ is again provided by the universal lower and upper bounds

$$
\omega_{\wedge}(\|x\|)=\frac{1-\|x\|}{1+\|x\|}, \quad \omega^{\wedge}\|x\|=\frac{1+\|x\|}{1-\|x\|}
$$

(cf. Proposition 3.8.5 and [6]). However, even for the one-dimensional case there are many examples when $\omega_{1}$ and $\omega_{2}$ can be improved and may be chosen more precisely (see examples below).

Let now $\gamma_{i}$ be functions defined on $(0,1)$ such that

$$
\frac{\gamma_{i}^{\prime}(s)}{\gamma_{i}(s)}=\frac{1}{s \omega_{i}(s)}, \quad i=1,2,
$$

i.e., $\ln \gamma_{i}(s)$ is a primitive function for $\omega_{i}(0) /\left(s \omega_{i}(s)\right), i=1,2$. Observe that, since the Cauchy problem (3.9.11) is globally solvable on $t \in[0, \infty)$, and its solution $\beta_{i}(t, s)$ goes to zero as $t$ tends to $\infty$, it follows that the integrals

$$
\int_{0}^{s} \frac{d t}{t \omega_{i}(t)}
$$


are divergent and tend to $\infty$. Hence $\gamma_{i}$ can be continuously extended to $[0,1)$ by $\gamma_{i}(0)=0$. In order that $\gamma_{i}$ be defined uniquely, we set, for example, $\gamma_{i}(1 / 2)=1$. Then it is an increasing function on $(0,1)$ by (3.9.14). Now (3.9.11) and (3.9.14) imply that

$$
\gamma_{i}\left(\beta_{i}(t, s)\right)=e^{-t} \gamma_{i}(s), \quad i=1,2 .
$$

Recalling (3.9.10) and using Lemma 3.8.4 as in the Hilbert space case, we obtain the inequalities

$$
\beta_{2}(t,\|x\|) \leq\|v(t, x)\| \leq \beta_{1}(t,\|x\|),
$$

which, in turn, will be used in the proof of the following assertion, where $\omega=\left(\omega_{1}, \omega_{2}\right)$.

Proposition 3.9.1. Let $X$ be a complex Banach space such that its open unit ball $D$ is homogeneous and let $f \in \operatorname{Hol}(D, X)$ be $\omega$-star-like on $D$, with $f(\tau)=0, \tau \in D$. Let $\gamma_{i}, i=1,2$, be the solutions of (3.9.14) defined above. Then

$$
\left\|A^{-1}\right\|^{-1} k(\|x\|) \gamma_{2}(\|x\|) \leq\left\|f\left(M_{-\tau}(x)\right)\right\| \leq K(\|x\|) \gamma_{1}(\|x\|)\|A\|,
$$

where $A=f^{\prime}(\tau)$,

$$
\begin{aligned}
& K(p):= \sup _{s \in[0, p]} \frac{s}{\gamma_{1}(s)}, \quad 0<p \leq 1, \\
& k(p):=\inf _{s \in[0, p]} \frac{s}{\gamma_{2}(s)}, \quad 0<p \leq 1,
\end{aligned}
$$

and $M_{-\tau}$ is an automorphism of $D$ which takes $\tau$ to 0 . In particular,

$$
f(D) \supset D_{r}
$$

where $D_{r}$ is the ball centered at the origin of radius

$$
r=\left\|A^{-1}\right\|^{-1} \lim _{s \rightarrow 1^{-}} \gamma_{2}(s) k(s) .
$$

Proof. Since $\gamma_{1}$ and $\gamma_{2}$ are increasing functions, by (3.9.15) and (3.9.16) we have

$$
\begin{aligned}
& \gamma_{1}(\|v(t, x)\|) \leq \gamma_{1}\left(\beta_{1}(t,\|x\|)\right)=e^{-t} \gamma_{1}(\|x\|), \\
& \gamma_{2}(\|v(t, x)\|) \geq \gamma_{2}\left(\beta_{2}(t,\|x\|)\right)=e^{-t} \gamma_{2}(\|x\|) .
\end{aligned}
$$

These inequalities and definitions (3.9.18) and (3.9.19) imply that

$$
e^{-t} \gamma_{2}(\|x\|) \cdot k(\|x\|) \leq\|v(t, x)\| \leq e^{-t} \gamma_{1}(\|x\|) \cdot K(\|x\|)
$$

because $\|v(t, x)\| \leq\|x\|$ by the Schwarz Lemma. Using now the fact that $\tilde{f}(x)=$ $A^{-1} f\left(M_{-\tau}(x)\right)=\lim _{t \rightarrow \infty} e^{t} v(t, x)$ (see Proposition 3.7.5), we get (3.9.17). Since $\widetilde{f}(D)=$ $A^{-1} f(D)$ we also obtain (3.9.20) from the left-hand inequality of (3.9.17).

REMARK. Observe that it follows by (3.9.7), (3.9.8) and (3.9.12) that $\omega_{1}(0) \leq 1$ while $\omega_{2}(0) \geq 1$. If the $\operatorname{limits}_{s \rightarrow 0^{+}} \gamma_{i}^{\prime}(s), i=1,2$, exist and are positive, then $K(p)$ in $(3.9 .18)$ is finite and $k(p)$ in (3.9.19) is positive. In this case $w_{i}(0), i=1,2$, must actually equal 1. Indeed, it follows by (3.9.14) that

$$
\begin{aligned}
& \gamma_{i}^{\prime}\left(0^{+}\right)=\lim _{s \rightarrow 0^{+}} \frac{\gamma_{i}(s)}{s}=\lim _{s \rightarrow 0^{+}} \gamma_{i}^{\prime}(s), \\
& \lim _{s \rightarrow 0^{+}} \frac{\gamma_{i}(s)}{s} \cdot \lim _{s \rightarrow 0^{+}} \omega_{i}(s)=\gamma_{i}^{\prime}\left(0^{+}\right) \cdot \omega_{i}(0), \quad i=1,2 .
\end{aligned}
$$


Hence $\omega_{i}(0)=1, i=1,2$. In other words, in this case we must find functions $w_{i}$ such that $w_{i}(0)=1, i=1,2$.

ExAmPle 1. For each star-like mapping $f \in \operatorname{Hol}(D, X)$, lower and upper admissible bounds $\omega_{i}(s), i=1,2$, can be chosen as follows:

$$
\omega_{1}(s)=\frac{1-c s}{1+c s} \quad \text { and } \quad \omega_{2}(s)=\frac{1+c s}{1-c s},
$$

for some $c \in[0,1]$. If $0 \leq c<1$, then $f$ is said to be strongly star-like (see, for example [19]). We have

$$
\gamma_{1}(s)=\frac{c s}{(1-c s)^{2}}, \quad \gamma_{2}(s)=\frac{c s}{(1+c s)^{2}}, \quad K(p)=k(p)=\frac{1}{c} .
$$

So, we get the growth and covering estimates

$$
\frac{\|x\|}{(1+c\|x\|)^{2}} \leq\left\|f\left(M_{-\tau}(x)\right)\right\| \leq \frac{\|x\|}{(1-c\|x\|)^{2}} .
$$

In particular,

$$
f(D) \supset D_{1 /(1+c)^{2}} .
$$

Corollary 3.9.2. Let $X$ be a complex Banach space such that its open unit ball is a homogeneous domain. Then the image of every star-like mapping (with respect to an interior point) covers the ball of radius $1 / 4$ centered at the origin.

Proof. Set $c=1$ in (3.9.24).

Note that the characteristic functions of many classical examples of star-like mappings have lower and upper bounds of the form

$$
\omega_{1,2}(s)=1 \mp \lambda s .
$$

In this case, solving the equations

$$
\frac{\gamma_{1,2}^{\prime}(s)}{\gamma_{1,2}(s)}=\frac{1}{s(1 \mp \lambda s)}
$$

we get $\gamma_{1,2}(s)=s /(1 \mp \lambda s)$ and $K(p)=k(p)=1$. Thus we obtain the improved estimates

$$
\frac{\|x\|}{1+\lambda\|x\|} \leq\|f(x)\| \leq \frac{\|x\|}{1-\lambda\|x\|} .
$$

Now let us consider some different situations.

ExAmple 2. Let $D=\Delta$ be the open unit disk in $\mathbb{C}$, and let $f(z)=z /(1-z)$. It is easy to see that $f$ is a convex, hence a star-like function, $f(0)=0$, and that its characteristic function is $g(z)=z-z^{2}$ (recall that $g(z)=f(z) / f^{\prime}(z)$ ). In this case $\omega_{i}(s), i=1,2$, can be chosen as $\omega_{1}(s)=1-s$ and $\omega_{2}(s)=1+s$.

Consequently, $\lambda=1$ and

$$
\frac{|z|}{1+|z|} \leq|f(z)| \leq \frac{|z|}{1-|z|}
$$

In particular, $f(\Delta) \supset \Delta_{1 / 2}$. 
ExAmple 3 . Let $D_{2}$ be the open unit ball in $\mathbb{C}^{2}, a \in \mathbb{C}$, and let $g_{a}: D_{2} \rightarrow \mathbb{C}^{2}$ be defined by

$$
g_{a}\left(z_{1}, z_{2}\right):=\left(z_{1}-a z_{2}^{2}, z_{2}\right)
$$

We know (see Example 1 after Proposition 3.8.6) that this mapping is a generator if $|a| \leq 3 \sqrt{3} / 2$ and that $\omega_{1,2}$ are of the form (3.9.25) with $\lambda=|a| \frac{2}{3 \sqrt{3}}$. If now $f_{a}$ is the star-like mapping corresponding to $g_{a}$, then we see that it satisfies the estimates (3.9.26).

EXAMPLE 4. In a similar way to Example 3 one can consider a more general case:

$$
g_{a, b}\left(z_{1}, z_{2}\right):=\left(z_{1}-a z_{2}^{2}, z_{2}-b z_{1}^{2}\right) .
$$

Now the question is for which $a$ and $b$ the mapping $g_{a, b}$ is a semicomplete vector field on

$$
D_{p}=\left\{\left(z_{1}, z_{2}\right) \in \mathbb{C}^{2}:\left|z_{1}\right|^{p}+\left|z_{2}\right|^{p}<1\right\} .
$$

We will give an answer for the cases $p=1$ and $p=3$ (for $p \neq 1,3$ the calculations are not so simple).

1. $p=1$. In this case a support functional $z^{*}$ at $z \in \mathbb{C}^{2}$ is given by

$$
\begin{aligned}
\left\langle w, z^{*}\right\rangle & =\left(w_{1} \frac{\left|z_{1}\right|}{z_{1}}+w_{2} \frac{\left|z_{2}\right|}{z_{2}}\right)\left(\left|z_{1}\right|+\left|z_{2}\right|\right), \\
\left\langle g_{a, b}(z), z^{*}\right\rangle & =\left(\left|z_{1}\right|+\left|z_{2}\right|-a z_{2}^{2} \frac{\left|z_{1}\right|}{z_{1}}-b z_{1}^{2} \frac{\left|z_{2}\right|}{z_{2}}\right)\left(\left|z_{1}\right|+\left|z_{2}\right|\right) .
\end{aligned}
$$

After some computations we get

$$
\|z\|^{2}(1-\lambda\|z\|) \leq \operatorname{Re}\left\langle g_{a, b}(z), z^{*}\right\rangle \leq\|z\|^{2}(1+\lambda\|z\|)
$$

where

$$
\|z\|=\left|z_{1}\right|+\left|z_{2}\right|, \quad \lambda=\frac{|a||b|}{|a|+|b|} .
$$

Thus $g_{a, b}$ is a semicomplete vector field if $1 /|a|+1 /|b| \geq 1$. (In fact, this condition is also necessary.) Therefore the star-like mapping corresponding to $g_{a, b}$ satisfies the estimates (3.9.26). In particular,

$$
\frac{\|z\|}{1+\|z\|} \leq\left\|f_{2,2}(z)\right\| \leq \frac{\|z\|}{1-\|z\|}, \quad \frac{2\|z\|}{2+\|z\|} \leq\left\|f_{1,1}(z)\right\| \leq \frac{2\|z\|}{2-\|z\|} .
$$

2. $p=3$. Now the support functional at $z \in \mathbb{C}^{2}$ is given by

$$
\left\langle w, z^{*}\right\rangle=\left(w_{1} \frac{\left|z_{1}\right|^{3}}{x_{1}}+w_{2} \frac{\left|z_{2}\right|^{3}}{z_{2}}\right) /\left(\left|z_{1}\right|^{3}+\left|z_{2}\right|^{3}\right)^{1 / 3}
$$

Consequently,

$$
\left\langle g_{a, b}(z), z^{*}\right\rangle=\frac{\left|z_{1}\right|^{3}+\left|z_{2}\right|^{3}-a z_{2}^{2} \frac{\left|z_{1}\right|^{3}}{z_{1}}-b z_{1}^{2} \frac{\left|z_{2}\right|^{3}}{z_{2}}}{\left(\left|z_{1}\right|^{3}+\left|z_{2}\right|^{3}\right)^{1 / 3}} .
$$

It is easy to observe that

$$
\|z\|^{2}\left(1-\frac{|a|+|b|}{2^{4 / 3}}\|z\|\right) \leq \operatorname{Re}\left\langle g_{a, b}(z), z^{*}\right\rangle \leq\|z\|^{2}\left(1+\frac{|a|+|b|}{2^{4 / 3}}\|z\|\right),
$$

where $\|z\|=\left(\left|z_{1}\right|^{3}+\left|z_{2}\right|^{3}\right)^{1 / 3}$. Therefore the mapping $g_{a, b}$ is a semicomplete vector field if $|a|+|b|<2^{4 / 3}$. (Again, this condition is also necessary.) The corresponding star-like mapping $f_{a, b}$ satisfies the estimate $(3.9 .26)$ with $\lambda=(|a|+|b|) / 2^{4 / 3}$. 
ExAmple 5. Consider the mapping $f: D_{2} \rightarrow \mathbb{C}^{2}$ defined by

$$
f_{1}(z):=\frac{z_{1}-z_{2}}{z_{1} e^{z_{1}}-z_{2} e^{z_{2}}} z_{1} e^{z_{1}}, \quad f_{2}(z):=\frac{z_{1}-z_{2}}{z_{1} e^{z_{1}}-z_{2} e^{z_{2}}} z_{2} e^{z_{2}} .
$$

Calculations show that $f$ is univalent on $D_{2}$ and

$$
g\left(z_{1}, z_{2}\right):=\left[f^{\prime}\left(z_{1}, z_{2}\right)\right]^{-1} f\left(z_{1}, z_{2}\right)=\left(z_{1}\left(1+z_{2}\right), z_{2}\left(1+z_{1}\right)\right)
$$

(cf. [19]).

Since $g: D_{2} \rightarrow \mathbb{C}^{2}$ is a semicomplete vector field, it follows that $f$ is star-like. In addition, in this case lower and upper bounds $\omega_{1}$ and $\omega_{2}$ can be chosen as in (3.9.25) with $\lambda=1 / \sqrt{2}$. Hence

$$
\frac{\|z\|}{1+\frac{1}{\sqrt{2}}\|z\|} \leq\|f(z)\| \leq \frac{\|z\|}{1-\frac{1}{\sqrt{2}}\|z\|}
$$

and $f\left(D_{2}\right)$ covers an open ball of radius $\sqrt{2} /(\sqrt{2}+1)$.

Acknowledgments. The first author was partially supported by the Rashi-Sakta Foundation. The second author was partially supported by the Fund for the Promotion of Research at the Technion and by the Technion VPR Fund.

\section{References}

[1] M. Abate, Converging semigroups of holomorphic maps, Atti Accad. Naz. Lincei Rend. Cl. Sci. Fis. Mat. Natur. 82 (1988), 223-227.

[2] - Iteration Theory of Holomorphic Maps on Taut Manifolds, Mediterranean Press, 1989.

[3] - , The infinitesimal generators of semigroups of holomorphic maps, Ann. Mat. Pura Appl. 161 (1992), 167-180.

[4] D. Abts, On injective holomorphic Fredholm mappings of index 0 in complex Banach spaces, Comment. Math. Univ. Carolin. 21 (1980), 513-525.

[5] D. Aharonov, M. Elin, S. Reich and D. Shoikhet, Parametric representations of semicomplete vector fields on the unit balls in $\mathbb{C}^{n}$ and Hilbert space, Atti Accad. Naz. Lincei 10 (1999), 229-253.

[6] D. Aharonov, S. Reich and D. Shoikhet, Flow invariance conditions for holomorphic mappings in Banach spaces, Math. Proc. Roy. Irish Acad. 99A (1999), 93-104.

[7] L. Aizenberg, S. Reich and D. Shoikhet, One-sided estimates for the existence of null points of holomorphic mappings in Banach spaces, J. Math. Anal. Appl. 203 (1996), 38-54.

[8] J. W. Alexander, Functions which map the interior of the unit circle upon simple regions, Ann. of Math. 17 (1915), 12-22.

[9] J. Arazy, An application of infinite dimensional holomorphy to the geometry of Banach spaces, in: Lecture Notes in Math. 1267, Springer, 1987, 122-150.

[10] D. K. Arrowsmith and C. M. Place, Ordinary Differential Equations: A Qualitative Approach with Applications, Chapman and Hall, London, 1982.

[11] V. Barbu, Nonlinear Semigroups and Differential Equations in Banach Spaces, Noordhoff, Leyden, 1976.

[12] R. W. Barnard, C. H. FitzGerald and S. Gong, The growth and 1/4-theorems for starlike mappings in $\mathbb{C}^{n}$, Pacific J. Math. 150 (1991), 13-22.

[13] A. Belleni-Morante and A. C. McBride, Applied Nonlinear Semigroups. An Introduction, Wiley, Chichester, 1998. 
[14] E. Berkson and H. Porta, Semigroups of analytic functions and composition operators, Michigan Math. J. 25 (1978), 101-115.

[15] H. Brézis, Opérateurs Maximaux Monotones, North-Holland, Amsterdam, 1973.

[16] L. Brickman, $\Phi$-like analytic functions, I, Bull. Amer. Math. Soc. 79 (1973), 555-558.

[17] H. Cartan, Sur la possibilité d'étendre aux fonctions de plusieurs variables complexes la théorie des fonctions univalentes, in [57], 129-155.

[18] C.-H. Chu and P. Mellon, Jordan structures in Banach spaces and symmetric manifolds, Exp. Math. 16 (1998), 157-180.

[19] M. Chuaqui, Applications of subordination chains to starlike mappings in $\mathbb{C}^{n}$, Pacific J. Math. 168 (1995), 33-48.

[20] C. C. Cowen and B. D. MacCluer, Composition Operators on Spaces of Analytic Functions, CRC Press, Boca Raton, FL, 1995.

[21] Yu. L. Daletskiı̌ and M. G. Krein, Stability of Solutions of Differential Equations in a Banach Space, Nauka, Moscow, 1970 (in Russian).

[22] S. Dineen, Complete holomorphic vector fields on the second dual of a Banach space, Math. Scand. 59 (1986), 131-142.

[23] —, The Schwarz Lemma, Clarendon Press, Oxford, 1989.

[24] S. Dineen, R. M. Timoney and J.-P. Vigué, Pseudodistances invariantes sur les domaines d'un espace localement convexe, Ann. Scuola Norm. Sup. Pisa 12 (1985), 515-529.

[25] P. Duren, Univalent Functions, Springer, Berlin, 1983.

[26] C. J. Earle and R. S. Hamilton, A fixed point theorem for holomorphic mappings, in: Proc. Sympos. Pure Math. 16, Amer. Math. Soc., Providence, RI, 1970, 61-65.

[27] M. Elin, S. Reich and D. Shoikhet, Holomorphically accretive mappings and spiral-shaped functions of proper contractions, Nonlinear Anal. Forum 5 (2000), 149-161.

[28] - , - - Dynamics of inequalities in geometric function theory, J. Inequal. Appl. 6 (2001), 651-664.

[29] T. Franzoni and E. Vesentini, Holomorphic Maps and Invariant Distances, North-Holland, Amsterdam, 1980.

[30] K. Goebel and S. Reich, Uniform Convexity, Hyperbolic Geometry and Nonexpansive Mappings, Dekker, New York, 1984.

[31] G. M. Golusin, Geometric Theory of Functions of a Complex Variable, Amer. Math. Soc., Providence, RI, 1969.

[32] S. Gong, Convex and starlike mappings in several complex variables, Science Press, Beijing, Kluwer, Dordrecht, 1999.

[33] A. W. Goodman, Univalent Functions, Vols. I, II, Mariner, Tampa, FL, 1983.

[34] K. R. Gurganus, $\Phi$-like holomorphic functions in $\mathbb{C}^{n}$ and Banach space, Trans. Amer. Math. Soc. 205 (1975), 389-406.

[35] L. A. Harris, Schwarz-Pick systems of pseudometrics for domains in normed linear spaces, in: Advances in Holomorphy, North-Holland, Amsterdam, 1979, 345-406.

[36] T. E. Harris, The Theory of Branching Processes, Springer, Berlin, 1963.

[37] L. F. Heath and T. J. Suffridge, Starlike, convex, close-to-convex, spirallike and $\Phi$-like maps in a commutative Banach algebra with identity, Trans. Amer. Math. Soc. 250 (1979), 195-212.

[38] U. Helmke and J. B. Moore, Optimization and Dynamical Systems, Springer, London, 1994.

[39] M. Hervé, Several Complex Variables: Local Theory, Tata Inst. Fund. Research, Bombay, and Oxford Univ. Press, London, 1963.

[40] —, Analyticity in Infinite Dimensional Spaces, de Gruyter, Berlin, 1989.

[41] J. M. Isidro and L. L. Stacho, Holomorphic Automorphism Groups in Banach Spaces: An Elementary Introduction, North-Holland, Amsterdam, 1984. 
[42] D. W. Jordan and P. Smith, Nonlinear Ordinary Differential Equations, Clarendon Press, Oxford, 1987.

[43] W. Kaplan, Close-to-convex schlicht functions, Michigan Math. J. 1 (1952), 169-185.

[44] W. Kaup, A Riemann mapping theorem for bounded symmetric domains in complex Banach spaces, Math. Z. 183 (1983), 503-529.

[45] V. Khatskevich, S. Reich and D. Shoikhet, Fixed point theorems for holomorphic mappings and operator theory in indefinite metric spaces, Integral Equations Operator Theory 22 (1995), 305-316.

[46] - - - -, Global implicit function and fixed point theorems for holomorphic mappings and semigroups, Complex Variables 28 (1996), 347-356.

[47] - , - - - Complex dynamical systems on bounded symmetric domains, Electronic J. Differential Equations 1997, No. 19, 1-9.

[48] - - - - , Asymptotic behavior of solutions of evolution equations and the construction of holomorphic retractions, Math. Nachr. 189 (1998), 171-178.

[49] V. Khatskevich and D. Shoikhet, Differentiable Operators and Nonlinear Equations, Birkhäuser, Basel, 1994.

[50] - - - One version of implicit function theorem for holomorphic mappings, C. R. Acad. Sci. Paris 319 (1994), 599-604.

[51] T. Kuczumov, S. Reich and D. Shoikhet, Fixed points of holomorphic mappings: a metric approach, in: Handbook of Metric Fixed Point Theory, Kluwer, Dordrecht, 2001, 437-515.

[52] - , - - , The existence and nonexistence of common fixed points for commuting families of holomorphic mappings, Nonlinear Anal. 43 (2001), 45-59.

[53] A. Lyzzaik, On a conjecture of M. S. Robertson, Proc. Amer. Math. Soc. 91 (1984), $108-110$.

[54] R. H. Martin, Jr., Differential equations on closed subsets of a Banach space, Trans. Amer. Math. Soc. 179 (1973), 399-414.

[55] T. Matsuno, On star-like theorems and convex-like theorems in the complex vector space, Sci. Rep. Tokyo, Kyoiku Daigaku 5 (1955), 88-95.

[56] I. Miyadera, Nonlinear Semigroups. An Introduction, Amer. Math. Soc., Providence, RI, 1992.

[57] P. Montel, Leçons sur les Fonctions Univalentes ou Multivalentes, Gauthier-Villars, Paris, 1933.

[58] R. Nevanlinna, Über die konforme Abbildung Sterngebieten, Oeversikt av FinskaVetenskaps Societeten Ferhandlinger 63(A), No. 6, 1921.

[59] A. Pazy, Semigroups of nonlinear contractions and their asymptotic behavior, in: Nonlinear Analysis and Mechanics, Pitman Res. Notes Math. 30, Longman, 1979, 36-134.

[60] J. A. Pfaltzgraff, Subordination chains and univalence of holomorphic mappings in $\mathbb{C}^{n}$, Math. Ann. 210 (1974), 55-68.

[61] - Subordination chains and quasiconformal extension of holomorphic maps in $\mathbb{C}^{n}$, Ann. Acad. Sci. Fen. Ser. A Math. 1 (1975), 13-25.

[62] J. A. Pfaltzgraff and T. J. Suffridge, Close-to-starlike holomorphic functions of several variables, Pacific J. Math. 57 (1975), 271-279.

[63] T. Poreda, On the univalent holomorphic maps of the unit polydisc in $\mathbb{C}^{n}$ which have the parametric representation I, II, Ann. Univ. Mariae Curie-Skłodowska 41 (1987), 105-113, $115-121$.

[64] —, On generalized differential equations in Banach space, Dissertationes Math. 310 (1991).

[65] S. Reich, Minimal displacement of points under weakly inward pseudo-lipschitzian mappings, Atti Accad. Naz. Lincei 59 (1975), 40-44.

[66] - On fixed point theorems obtained from existence theorems for differential equations, J. Math. Anal. Appl. 54 (1976), 26-36. 
[67] S. Reich and D. Shoikhet, Generation theory for semigroups of holomorphic mappings in Banach spaces, Abstr. Appl. Anal. 1 (1996), 1-44.

[68] - - - Semigroups and generators on convex domains with the hyperbolic metric, Atti Accad. Naz. Lincei 8 (1997), 231-250.

[69] —, -, The Denjoy-Wolff theorem, Ann. Univ. Mariae Curie-Skłodowska 51 (1997), 219240.

[70] —, - Metric domains, holomorphic mappings and nonlinear semigroups, Abstr. Appl. Anal. 3 (1998), 203-228.

[71] M. S. Robertson, On the theory of univalent functions, Ann. of Math. 37 (1936), 374-408.

[72] - Applications of the subordination principle to univalent functions, Pacific J. Math. 11 (1961), 315-324.

[73] - Univalent functions starlike with respect to a boundary point, J. Math. Anal. Appl. 81 (1981), 327-345.

[74] B. A. Sevastyanov, Branching Processes, Nauka, Moscow, 1971 (in Russian).

[75] H. Silverman and E. M. Silvia, Subclasses of univalent functions starlike with respect to a boundary point, Houston J. Math. 16 (1990), 289-299.

[76] L. Špaček, Přispěvek k teorii funkci prostych, Časopis Pěst. Mat. 62 (1933), 12-19.

[77] E. Study, Konforme Abbildung Einfachzusammenhängender Bereiche, B. G. Teubner, Leipzig and Berlin, 1913.

[78] T. J. Suffridge, The principle of subordination applied to functions of several variables, Pacific J. Math. 33 (1970), 241-248.

[79] —, A holomorphic function having a discontinuous inverse, Proc. Amer. Math. Soc. 31 (1972), 629-630.

[80] -, Starlike and convex maps in Banach space, Pacific J. Math. 46 (1973), 575-589.

[81] —, Starlikeness, convexity and other geometric properties of holomorphic maps in higher dimensions, in: Complex Analysis (Proc. Conf. Univ. Kentucky, Lexington, KY, 1976), Lecture Notes in Math. 599, Springer, 1977, 146-159.

[82] H. Upmeier, Jordan Algebras in Analysis, Operator Theory and Quantum Mechanics, CBMS Reg. Conf. Ser. in Math., 67, Amer. Math. Soc., Providence, RI, 1987.

[83] E. Vesentini, Semigroups of holomorphic isometries, Adv. Math. 65 (1987), 272-306.

[84] -, Krein spaces and holomorphic isometries of Cartan domains, in: Geometry and Complex Variables, Marcel Dekker, New York, 1991, 409-413.

[85] —, Semigroups of holomorphic isometries, in: Complex Potential Theory, Kluwer, Dordrecht, 1994, 475-548.

[86] J. K. Wald, On Starlike Functions, Ph.D. thesis, Univ. of Delaware, Newark, DE, 1978. 